Portland State University

PDXScholar

Spring 5-24-2017

\title{
Investigation into Effects of Instability and Reactivity of Hydride-Passivated Silicon Nanoparticles on Interband Photoluminescence
}

Christine Marie Radlinger

Portland State University

Follow this and additional works at: https://pdxscholar.library.pdx.edu/open_access_etds

Part of the Nanoscience and Nanotechnology Commons

Let us know how access to this document benefits you.

\section{Recommended Citation}

Radlinger, Christine Marie, "Investigation into Effects of Instability and Reactivity of Hydride-Passivated Silicon Nanoparticles on Interband Photoluminescence" (2017). Dissertations and Theses. Paper 3619. https://doi.org/10.15760/etd.5511

This Dissertation is brought to you for free and open access. It has been accepted for inclusion in Dissertations and Theses by an authorized administrator of PDXScholar. Please contact us if we can make this document more accessible: pdxscholar@pdx.edu. 
Investigation into Effects of Instability and Reactivity of Hydride-Passivated Silicon Nanoparticles on Interband Photoluminescence

by

Christine Marie Radlinger

A dissertation submitted in partial fulfillment of the requirements for the degree of

Doctor of Philosophy

in

Chemistry

Dissertation Committee:

Andrea Goforth, Chair

Theresa McCormick

Mark Woods

Rolf Koenenkamp

Portland State University

2017 


\begin{abstract}
While silicon has long been utilized for its electronic properties, its use as an optical material has largely been limited due to the poor efficiency of interband transitions. However, discovery of visible photoluminescence (PL) from nanocrystalline silicon in 1990 triggered many ensuing research efforts to optimize PL from nanocrystalline silicon for optical applications. Currently, use of photoluminescent silicon nanoparticles (Si NPs) is commercially limited by: 1) the instability of the energy and intensity of the PL, and 2) the low quantum yield of interband PL from Si NPs.

Herein, red-emitting, hydrogen-passivated silicon nanoparticles (H-Si NPs) were synthesized by thermally-induced disproportionation of a $\mathrm{HSiCl}_{3}$-derived $\left(\mathrm{HSiO}_{1.5}\right) \mathrm{n}$ polymer. The H-Si NPs produced by this method were then subjected to various chemical and physical environments to assess the long-term stability of the optical properties as a function of changing surface composition. This dissertation is intended to elucidate correlations between the reported PL instability and the observed changes in the Si NP surface chemistry over time and as a function of environment.
\end{abstract}

First, the stability of the H-Si NP surface at slightly elevated temperatures towards reactivity with a simple alkane was probed. The H-Si NPs were observed by FT-IR spectroscopy to undergo partial hydrosilylation upon heating in refluxing hexane, in addition to varying degrees of surface oxidation. The unexpected reactivity of the $\mathrm{Si}$ surface in $n$-hexane supports the unstable nature of the H-Si NP surface, and furthermore implicates the presence of highly-reactive Si radicals on the surfaces of the Si NPs. We propose that reaction of alkene impurities with the $\mathrm{Si}$ surface radicals is largely responsible for the observed surface alkylation. However, we also present an alternate 
mechanism by which Si surface radicals could react with alkanes to result in alkylation of the surface.

Next, the energy and intensity stability of the interband PL from H-Si NPs in the presence of a radical trap was probed. Upon addition of (2,2,6,6,-tetramethyl-piperidin-1yl)oxyl (TEMPO), the energy and intensity of the interband transition was observed to change over time, dependent on the reaction conditions. First, when the reaction occurred at $4^{\circ} \mathrm{C}$ with minimal light exposure, the interband transition exhibited a gradual hypsochromic shift to between $595 \mathrm{~nm}$ and $655 \mathrm{~nm}$, versus the $\lambda_{\max }$ of the original low energy emission peak at $700 \mathrm{~nm}$, depending on the amount of TEMPO in the sample. Second, when the reaction proceeded at room temperature with frequent exposure to $360 \mathrm{~nm}$ irradiation, the original interband transition at $660 \mathrm{~nm}$ was quenched while a new peak at $575 \mathrm{~nm}$ developed. Based on all the data collected and analyzed, we assign the $595-655 \mathrm{~nm}$ transition as due to interband exciton recombination from Si NPs with reduced diameters relative to the original Si NPs. We furthermore assign the $575 \mathrm{~nm}$ transition as due to an oxide-related defect state resulting from rapid oxidation of photoexcited Si NPs. 


\section{Dedication}

For dad, mom, and Joseph. You never said I couldn't. 


\section{Acknowledgements}

I would like to express my deepest gratitude to my research advisor, Dr. Andrea Goforth. Your unyielding faith in me, which I did not always understand at the time, has been an immense factor in my achievements. I would not be the scientist that I am today without you. Thank-you from the bottom of my heart.

I would also like to thank my committee members, Drs. Theresa McCormick, Mark Woods, and Rolf Koenenkamp. Your combined experiences and vast library of knowledge has helped shape my research project throughout the years. Thank-you sincerely for your mentorship.

I would be remiss if I did not acknowledge my eleventh grade chemistry teacher, Ms. Steinmetz. She changed my course in life without even realizing it. Thank-you for convincing me that I, a young girl from small town Wisconsin, was smart enough to study science. From all of us who forgot to say so, "thank-you" for everything.

Finally, to my long-term investor. My life is much more full as a result of you being in it. You inspire me to push myself beyond my self-imposed limits, both as a professional and as an adventurer. On this day of all days, I am incredibly excited to see all that life has in order for us. Olive juice. 


\section{Table of Contents}

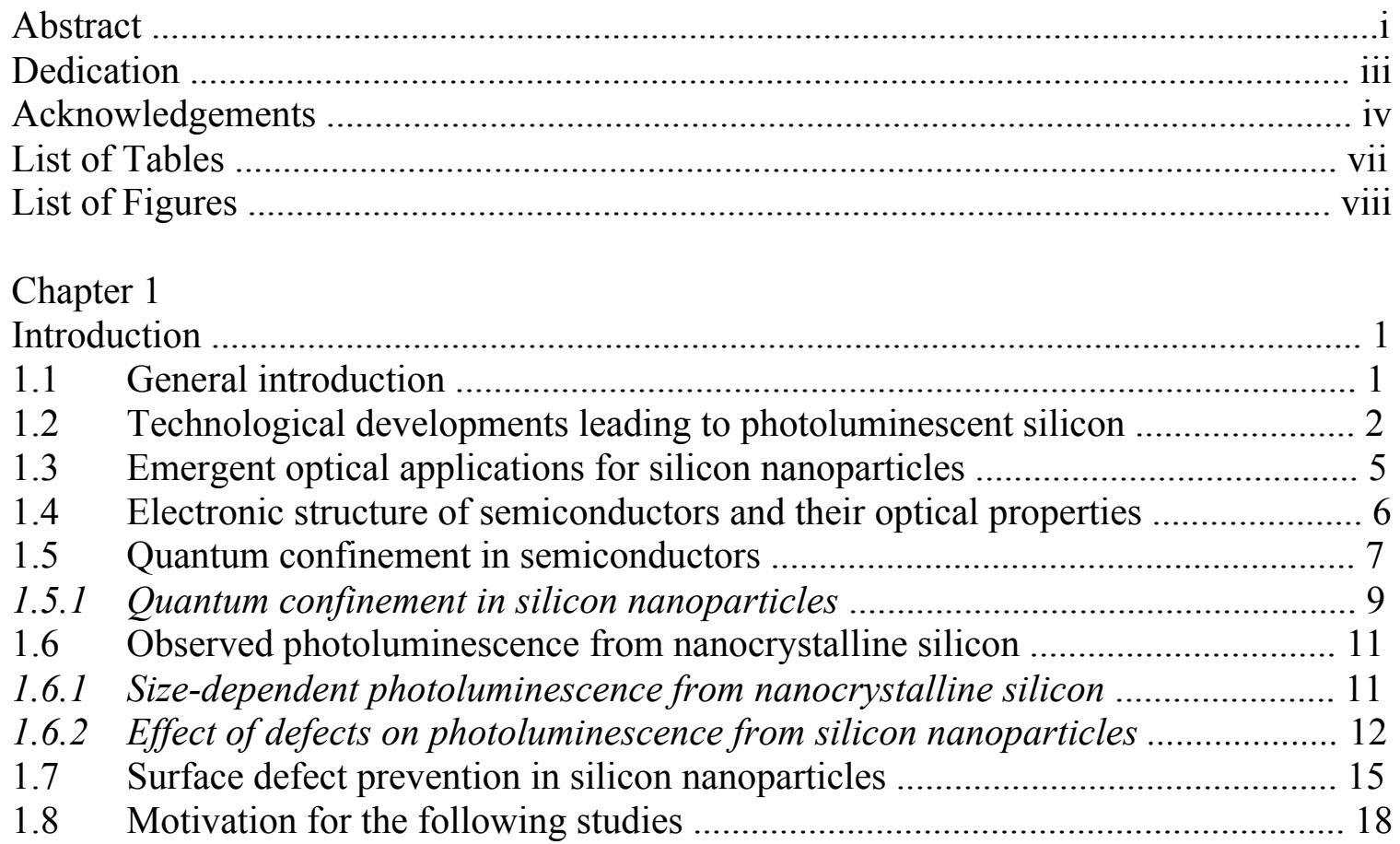

Chapter 2

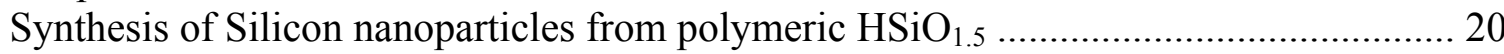

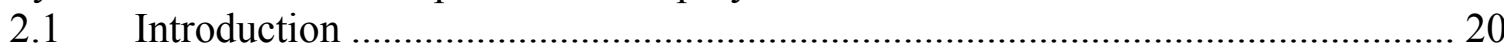

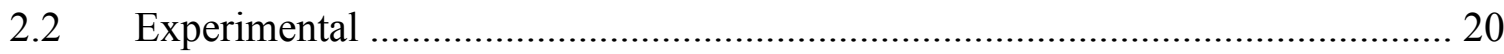

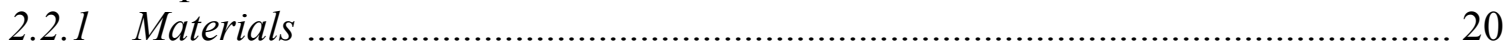

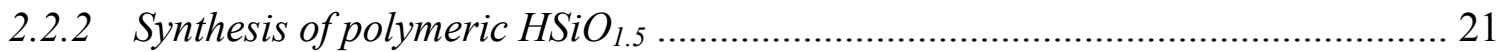

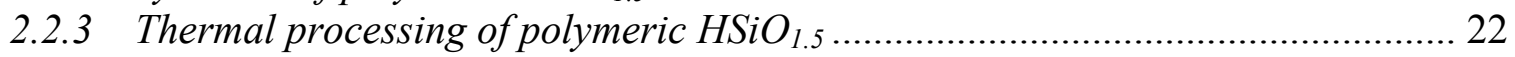

2.2.4 Liberation of Si NPs from oxide matrix …………......................................... 23

2.2.5 Fourier-transform infrared (FT-IR) spectroscopy ............................................ 24

2.2.6 X-ray photoelectron spectroscopy (XPS) ………………............................. 25

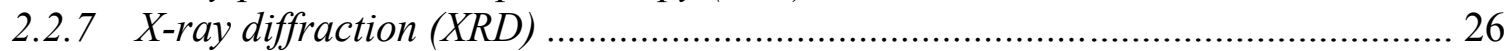

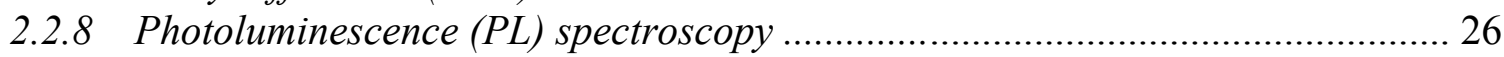

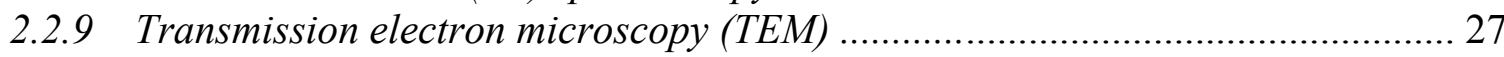

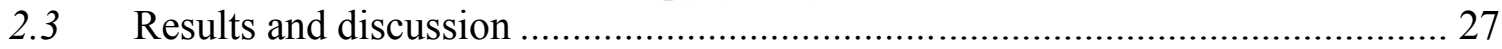

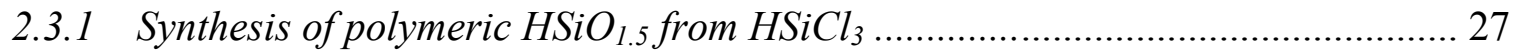

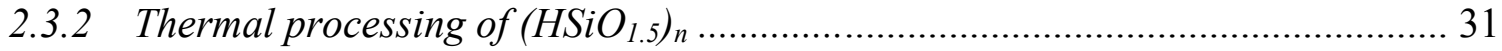

2.3.3 Liberation of Si NPs from oxide matrix......................................................... 38

Chapter 3

Hydrosilylation of silicon nanoparticles in $n$-alkane solvents:

implication of radicals in the chemical reactivity of hydride-passivated surfaces .......... 43

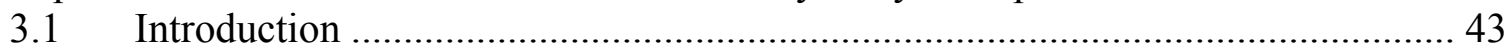

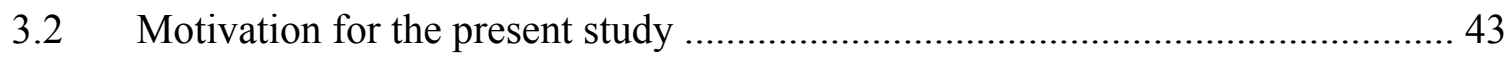

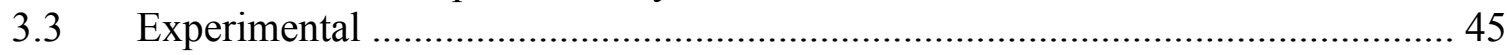




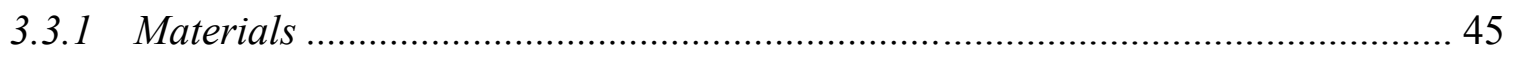

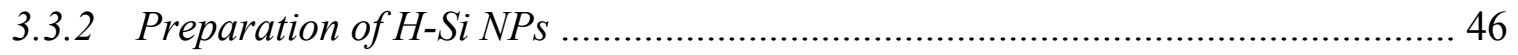

3.3.3 Purification of partially-oxidized H-Si NPS .................................................. 47

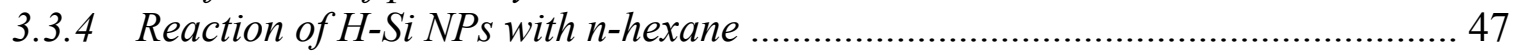

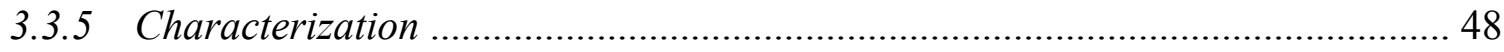

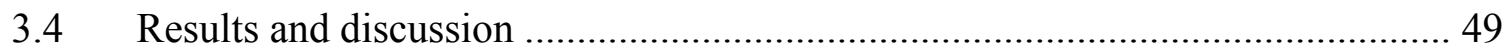

3.4.1 FT-IR characterization of Si NPs reacted with $n$-hexane ..................................5 50

3.4.2 UV-vis characterization of Si NPs reacted with purified n-hexane ..................... 55

3.4.3 Photoluminescence characterization of Si NPs reacted with $n$-hexane ............... 55

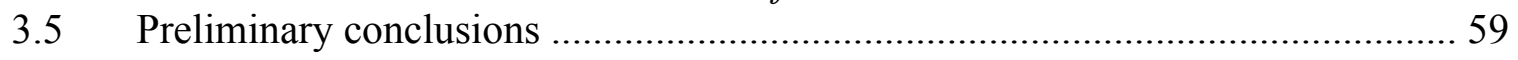

Chapter 4

Investigation into the effect of (2,2,6,6-tetramethyl-piperidin-1-yl)oxyl radical on silicon

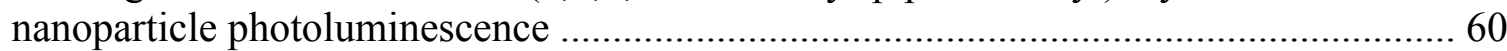

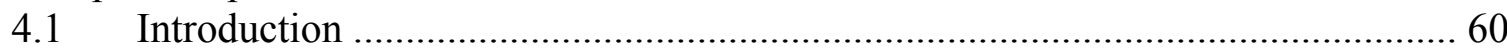

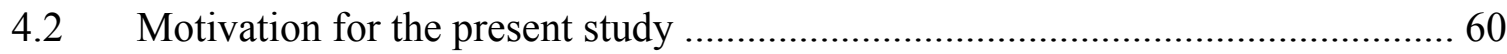

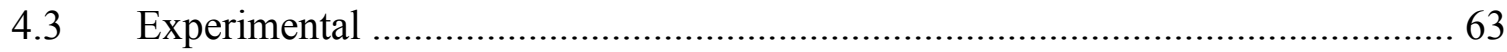

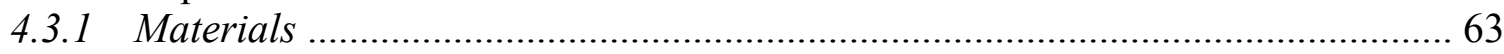

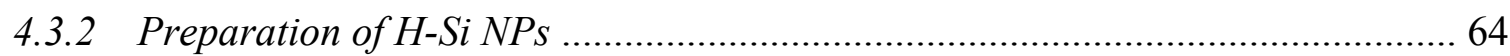

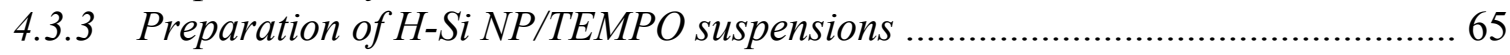

4.3.4 Scale-up of 8.0 mM TEMPO sample for chemical analysis ................................ 67

4.3.5 Photoluminescence characterization of Si NP/TEMPO suspensions ................... 67

4.3.6 Chemical analysis of large-scale 8.0 mM TEMPO sample ................................. 69

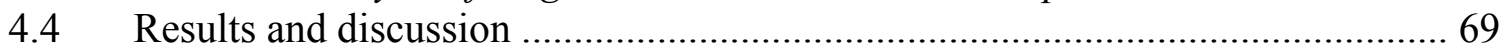

4.4.1 Reaction of H-Si NPs with TEMPO: effect of time on PL (study one) ................ 70

4.4.2 Reaction of H-Si NPs with TEMPO: closer look at initial brightening

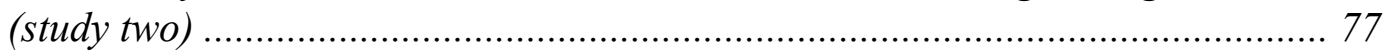

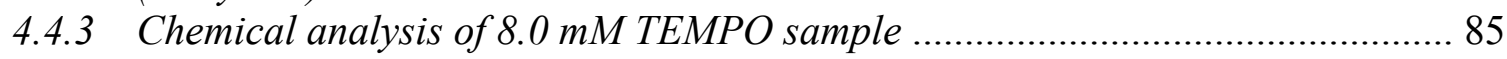

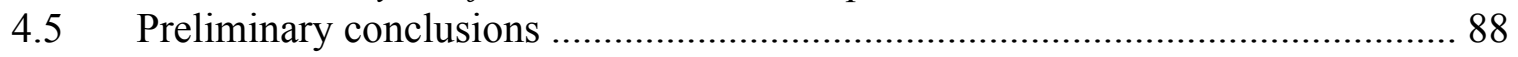

Chapter 5

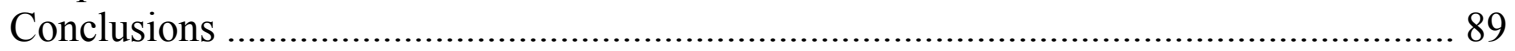

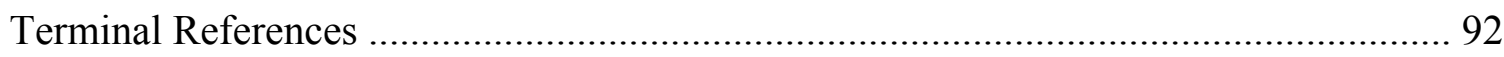

Appendix

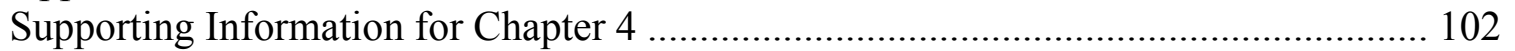




\section{List of Tables}

Table 2.1. Effect of annealing temperature on Si NP size and crystallinity .................. 33

Table 2.2. Effect of annealing time on Si NP size and crystallinity ............................ 34

Table 4.1. Sample preparation parameters for study one ......................................... 70

Table 4.2. Sample preparation parameters for study two ......................................... 78 


\section{List of Figures}

Figure 1.1 Schematic of the first point-contact transistor ......................................... 3

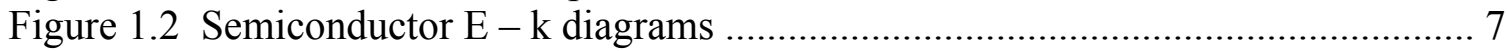

Figure 1.3 Schematic of density of states for bulk $v$ s. nanocrystal semiconductor ......... 8

Figure 1.4 Graphical representation of EMA for Si NP bandgap energy .................... 10

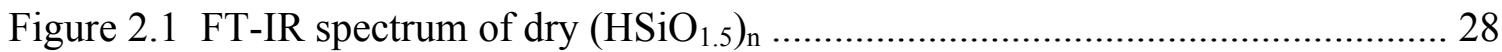

Figure 2.2 XPS survey scan of dry polymer .......................................................... 29

Figure 2.3 XPS spectrum of Si 2p region for dry polymer ...................................... 30

Figure 2.4 XRD patterns of polymer annealed for $10 \mathrm{~h}$ at varying temperatures .......... 32

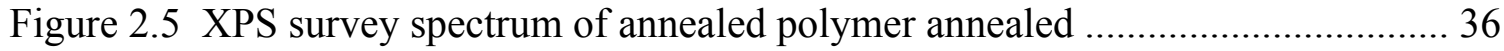

Figure 2.6 XPS spectrum of Si 2p region of annealed polymer .................................. 37

Figure 2.7 FT-IR spectrum of H-Si NPs ............................................................. 39

Figure 2.8 PL and PLE spectra of H-Si NPs ....................................................... 40

Figure 2.9 TEM images of H-Si NP aggregate structures ....................................... 41

Figure 3.1 FT-IR spectra of Si NPs before and after reaction with $n$-hexane ............... 51

Figure 3.2 UV-vis spectra of Si NPs before and after reaction with $n$-hexane .............. 55

Figure 3.3 PL and PLE spectra of Si NPs before and after reaction with $n$-hexane ...... 56

Figure 4.1. Digital images of samples from study 1 at $\mathrm{t}=5$ and $90 \mathrm{~min}$..................... 71

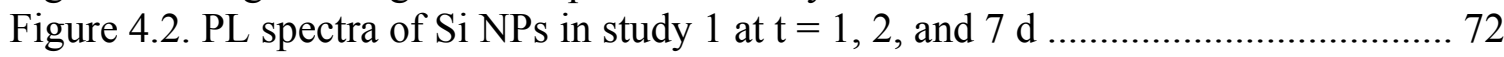

Figure 4.3. Plot showing shift in low energy PL over time ..................................... 74

Figure 4.4. Digital images of samples from study 1 at $\mathrm{t}=1,2$, and $7 \mathrm{~d}$....................... 76

Figure 4.5. PL spectra of Si NPs in study 2 (low energy PL) ..................................... 79

Figure 4.6. Stern-Volmer plot of PL quenching by TEMPO ..................................... 81

Figure 4.7. PL and PLE spectra of Si NPs from study 2 at $\mathrm{t}=0$ and $24 \mathrm{~h}$.................... 84

Figure 4.8. FT-IR spectra of H-Si NPs before and after reaction with TEMPO ............ 87

Figure 5.1. Energy diagram for unoxidized H-Si NPs ............................................ 89

Figure 5.2. Energy diagram for oxidized Si NPs .................................................. 91 
Chapter 1

Introduction

\subsection{General introduction}

A long-standing trend in the development of electronics has been the miniaturization of individual components to produce more efficient devices, both in terms of the speed of signal transfer and the resources required for their manufacture. Concurrently, there has been a rapid increase in research into the production of inorganic nanomaterials due to their distinctively useful optical properties, such as size-tunable absorption and emission energies. ${ }^{1-4}$ Given their potential to improve optical and electronic devices, and enable new technologies, inorganic nanomaterials have become of interest in research labs across a wide range of disciplines. Synthetic control over the shape, size uniformity, and surface chemistry of these nanomaterials is fundamental to the design of next-generation technologies. Silicon ( $\mathrm{Si}$ ) nanostructures are an example of inorganic nanomaterials whose size-dependent visible photoluminescence (PL) has piqued the interest of scientists since the early 1990s. Since then, an immense amount of effort has gone into establishing synthetic control in order to realize their size-tunable properties.

$\mathrm{Si}$ has long been the semiconductor of choice in the fabrication of microprocessors and other electronic devices. This is due in part to its ease of processing relative to other semiconductors (e.g., germanium $(\mathrm{Ge}))$, as well as its high-quality native surface-oxide layer that acts as excellent insulator, a necessary component of modern transistors. ${ }^{5}$ Direct transitions between the conduction and valence band extrema are not possible in Si. Transitions between the extrema require participation of a phonon. As a 
consequence, optical emission and absorption across the bandgap are weak. Nonetheless, nanocrystalline $\mathrm{Si}$ overcomes some of the limitations of its bulk counterpart due to changes in the electronic band structure when one or more of the crystallite dimensions are on the nanometer length scale. Specifically, nanocrystalline $\mathrm{Si}$ is observed to be optically active when the dimensions are reduced to a few nanometers. Furthermore, the bandgap energy $\left(E_{g}\right)$ is expected to be dependent on the size of the crystallite domains and therefore tunable. ${ }^{6-9}$ The size-dependence of the optical properties of $\mathrm{Si}$ on the nanometer length scale heighten the potential for the development of all Si-based optoelectronic devices in which signals would be transferred by photopulses (i.e., light) generated by the nanocrystalline Si diodes. ${ }^{10-13}$ Currently, the development of this type of technology is largely limited by the low efficiency and instability of the PL of Si nanomaterials. This low efficiency is thought to be caused in part by a variety of electronically active energy states belonging to defects that develop on the Si surface, the presence of which are expected to reduce the yield of radiative interband transitions. This dissertation studies the effect of the instability of the hydride-passivated silicon nanoparticle (H-Si NP) surface on the observed optical properties. Ideally, by obtaining a better understanding of the conditions of formation and chemical reactivity of the defects present on the H-Si NP surface, one could envision being able to mitigate the reactivity of the Si NP surface and, as a result, optimize the optical performance of the material.

\subsection{Technological developments leading to photoluminescent silicon}

In late 1947, Drs. Bardeen, Brattain, and Shockley created the first successful semiconductor amplifier, which operated based on the principles of the transistor effect. 


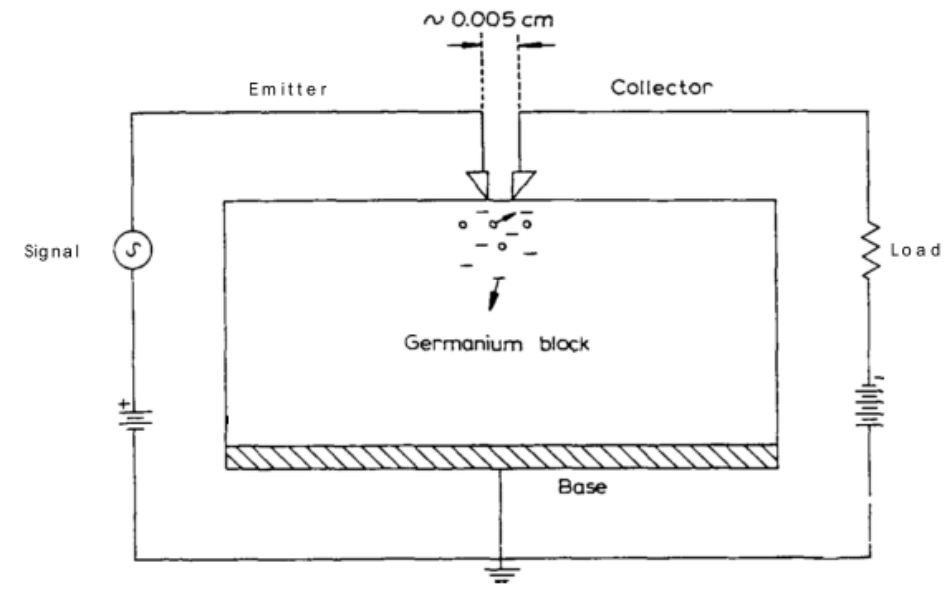

Figure 1.1. Schematic of the first point-contact transistor. ${ }^{14}$

This new technology quickly replaced vacuum tube amplifiers owing to its low energy cost of operation and the transistor is now one of the most ubiquitous technologies in devices produced worldwide. The original device consisted of a single-crystal of n-type Ge with two metallic point contacts located within $0.005 \mathrm{~cm}$ of one another on the surface of the crystal, and a larger contact at the base. They discovered that when a small positive bias was applied on the emitter contact and a negative bias on the collector contact, small changes to the emitter current resulted in an amplification of the collector current. A schematic of the first point-contact transistor is shown in Figure 1.1. ${ }^{14}$

In the mid-1950s, shortly following the invention of the Ge point contact transistor, researchers at Bell Telephone Laboratories (Bell Labs) began to investigate ways to replace Ge with $\mathrm{Si}^{15,16}$ One driving factor for doing so was the higher natural abundance of $\mathrm{Si}$, and therefore lower cost of $\mathrm{Si}$ relative to Ge. In addition, Si crystals exhibited higher stabilities at elevated temperatures versus Ge, which would prove to be crucial in the role of transistors. ${ }^{5}$ Optimization of the electronic performance of the $\mathrm{Si}$ crystals required the development of methods for removing defects from the crystal 
surface prior to device construction. Anodic polishing was the standard technique for doing so with Ge crystals; therefore, the technique was modified to remove defects from Si crystals as well.

In anodic polishing of a Group IV wafer, the semiconductor crystal serves as the anode and is placed in an electrolytic solution containing hydrofluoric acid (HF) and a platinum cathode. A current is passed through the cell to result in the formation of an anodic film on the surface of the crystal. ${ }^{17}$ The layer that is formed on the surface of the crystal is slightly soluble in the electrolytic solution, and therefore slowly dissolves over time to expose new layers of atoms. Using anodic polishing, the researchers at Bell Labs were successful in the preparation of atomically smooth p-type Si surfaces in concentrated solutions of HF if a threshold current density of $0.01-0.1 \mathrm{~A} / \mathrm{cm}^{2}$ was exceeded on the surface of the crystal. ${ }^{16}$ Additionally, they observed that the current densities necessary to polish p-type Si were not sufficient for n-type Si. Further studies into the etching mechanism of n-type Si suggested that below a threshold current density, the process was actually causing a thin mesoporous film to form on the crystal surface. ${ }^{17}$ At the time, it was determined by electron and X-ray diffraction that the surface was amorphous, and that the outermost layer was made up of divalent Si atoms. It was further concluded that strong UV-illumination would be required in order to polish n-type $\mathrm{Si}$, rather than etch the surface.

Developments in transmission electron microscopy (TEM) prior to the $1980 \mathrm{~s}$ eventually made it possible to study the morphology of the polished wafer surfaces. TEM analysis of the surfaces confirmed that the n-type $\mathrm{Si}$ wafer was pitted rather than smooth. ${ }^{18}$ On closer inspection, it was observed to be composed of a mesoporous network 
containing nanoscale $\mathrm{Si}$ domains, and the material was called porous $\mathrm{Si}$ (p-Si) by the scientific community from that point forward. However, it was not until 1990 that intense PL from p-Si was first reported. ${ }^{19}$ The visible PL was thought to occur due to twodimensional size effects in the nanoscale Si domains, which result in an increase in the bandgap of Si versus its bulk value. Since that first report of visible PL from nanoscale Si domains, the field of Si nanomaterials has grown exponentially and now includes studies of ultra-small, colloidal Si nanoparticles (Si NPs) in variety of applications. Solid-, ${ }^{20-23}$ solution-, ${ }^{24-29}$ and gas-phase ${ }^{30-33}$ reductions of Si precursors are methods currently used to synthesize Si NPs. Extensive descriptions of the synthesis methodologies published to date have been reviewed elsewhere. ${ }^{34,35}$

\subsection{Emergent optical applications for silicon nanoparticles}

Numerous applications have been proposed for photoluminescent nanocrystalline $\mathrm{Si}$, most of which take advantage of the theoretically size-tunable optical absorption and emission capabilities. For example, both p-Si and Si NPs have been demonstrated as fluorescent sensors to detect a variety of organic compounds, such as formaldehyde and nitro-functionalized aromatic compounds. ${ }^{36-39}$ In addition, Si NPs have been envisioned as replacements for traditional organic dyes in a variety of sensing and tracking applications owing to their strong visible PL and superior resistance to photobleaching and photodegredation..$^{22,23,40-42}$ For biomedical imaging and sensing applications, red-toNIR-fluorescence is desired, since these wavelengths penetrate tissue, and do not damage cells. While the number of red-to-NIR-emitting molecular organic dyes is limited (e.g., to cyanine and rhodamine dyes and some of their derivatives), protocols for synthesizing 
red-fluorescent Si NPs are well established. ${ }^{21,23,43}$ Another intended application for emissive Si NPs is in optoelectronic devices. Currently, modern optoelectronic devices use III-V semiconductor nanoparticles as the light-emitting diodes, which are then interfaced to the electronic silicon-based circuitry. However, the lattice mismatch that occurs between silicon, the main component in electronics, and the III-V diode materials substantially increases the difficulty of device fabrication. Therefore, the ability to use silicon as the light-emitting diode material would potentially make optoelectronic device fabrication much easier, and is a focus of much silicon nanomaterial research. ${ }^{10-13,44}$

Difficulties involved with integration of nanocrystalline Si into commercial devices arise in stabilizing the PL intensity and energy in aqueous or aerobic environments. $^{23,41,45}$ Changes in the PL of nanocrystalline Si over time, presumably alongside alterations in the surface chemistry or size of the particles, have been reported consistently across the literature..$^{23,30,46}$ However, the promise of robust, inorganic fluorophores with size-dependent optical properties continues to motivate researchers to develop strategies to mitigate the changing PL energy and intensity.

\subsection{Electronic structure of semiconductors and their optical properties}

Semiconductors can be sorted into two groups: direct bandgap and indirect bandgap materials. In direct bandgap semiconductors, such as cadmium selenide (CdSe) or lead sulfide $(\mathrm{PbS})$, the valence and conduction band extrema are aligned along the $\mathrm{k}$-axis $(\Delta \mathrm{k}=0)($ Figure 1.2A). This means that there is no required change in momentum for charge carriers undergoing interband transitions, and therefore direct bandgap materials exhibit strong optical transitions. In contrast, the valence and conduction band 

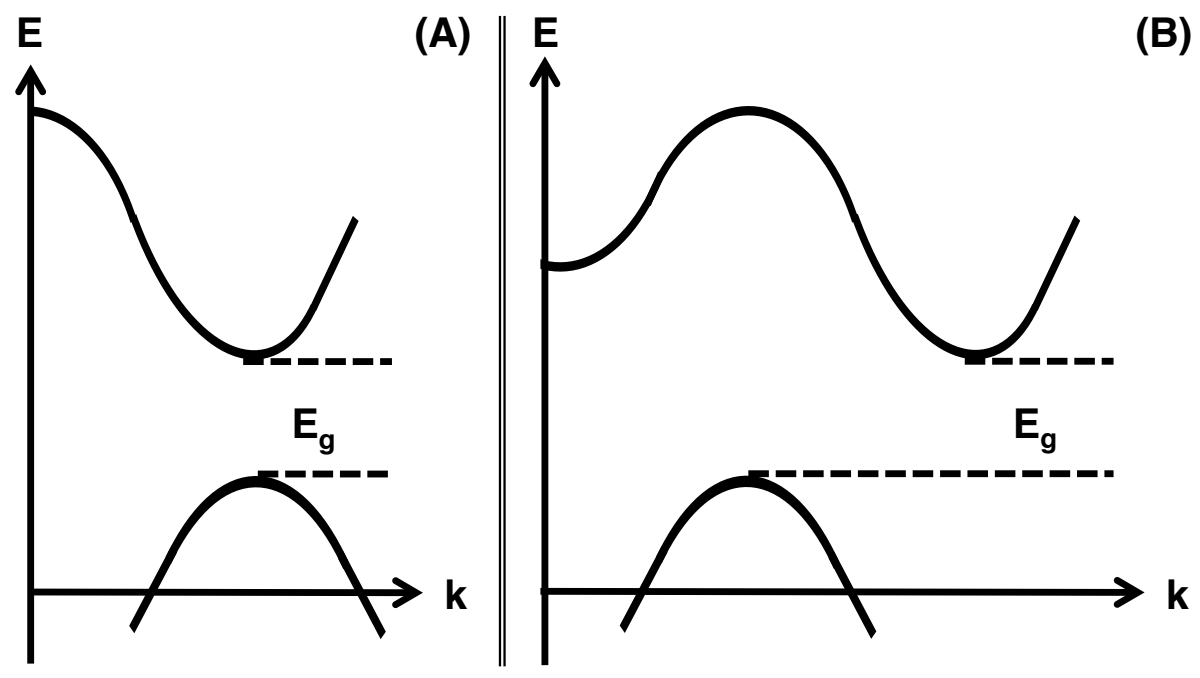

Figure 1.2. Simplified E - $\mathrm{k}$ diagrams for (A) direct bandgap and (B) indirect bandgap semiconductors. The lowest energy transition is indicated as $\mathrm{E}_{\mathrm{g}}$ in both panels.

extrema are not aligned along the $\mathrm{k}$-axis $(\Delta \mathrm{k} \neq 0)$ in indirect bandgap semiconductors (Figure 1.2B). Because of this, photons do not have enough momentum to excite an electron across the indirect bandgap alone. In order for transitions across an indirect bandgap to occur, they must be coupled with phonons (i.e., lattice vibrations), which have enough momentum to elicit the transition. Indirect bandgap materials are therefore exhibit weak optical transitions. As a result, the radiative rates of interband transitions for direct bandgap semiconductors are much greater than the radiative rates of interband transitions for indirect bandgap semiconductors.

\subsection{Quantum confinement in semiconductors}

As the size of either a direct or an indirect bandgap semiconductor is reduced to nanoscale domains, the electronic structure deviates from that of the bulk material. When the dimensions approach the Bohr exciton radius of the material, size-dependent 


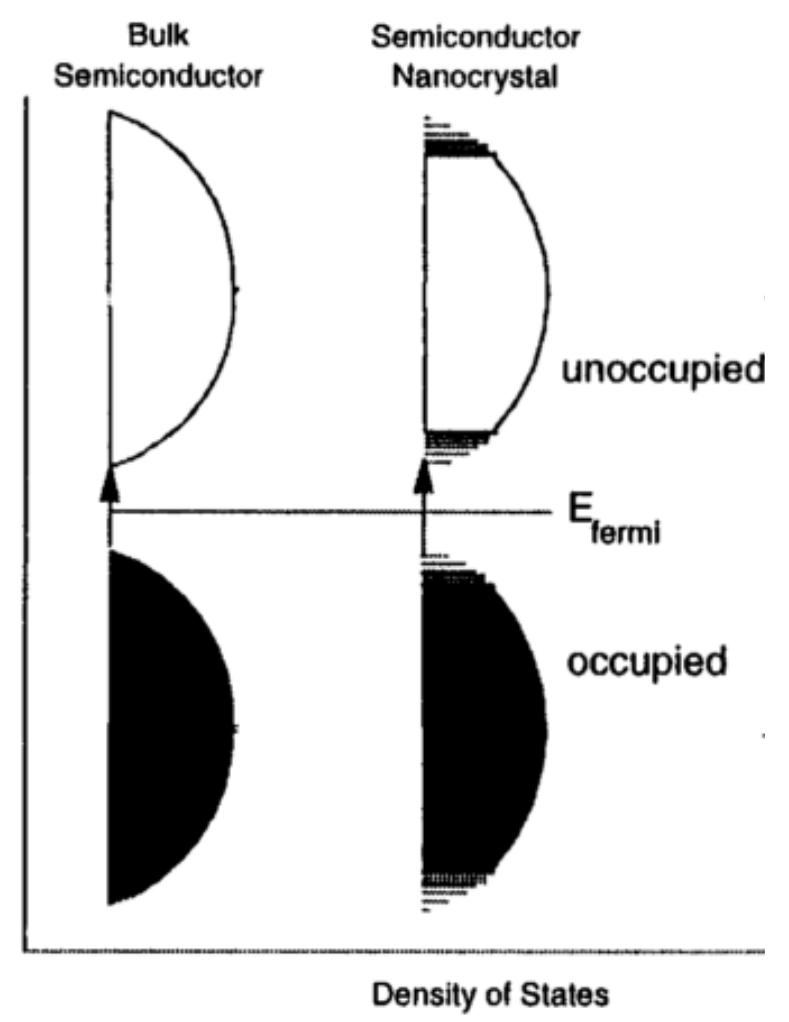

Figure 1.3. Schematic showing the effect of size reduction on the density of states of a semiconductor. Adapted with permission from (J. Phys. Chem. 1996, 100, 13226-13239). Copyright (C) 1996, American Chemical Society.

electronic properties are expected. ${ }^{7,11}$ As the ratio of surface-to-interior atoms increases with decreasing size, the density of states (DoS) of atomic orbitals at the band edges decreases, and discrete electronic states develop at the band edges, as shown in Figure $1.3{ }^{11}$ As the crystallite size is further reduced, elimination of atomic orbitals from the band edges results in an increase in the energy between the valence and conduction band extrema. The inverse relationship between crystallite size and bandgap energy $\left(\mathrm{E}_{\mathrm{g}}\right)$ is referred to as the quantum confinement $(\mathrm{QC})$ effect, and can be exploited for the synthesis of semiconductor NPs with specific PL energies. 


\subsubsection{Quantum confinement in silicon nanoparticles}

To date, numerous protocols have been developed for the size-selective, and therefore PL-energy selective, synthesis of direct bandgap semiconductor nanoparticles, which are also called quantum dots (QDs). ${ }^{4,47}$ The use of PbS, CdSe, and other QDs in cellular imaging, electronic devices and photovoltaic systems has been proposed, and in some cases achieved commercially. ${ }^{4}$ However, concerns regarding the toxicities of these QDs have been established in the literature. ${ }^{48} \mathrm{Si}$ NPs have been proposed as a potentially less-toxic alternative to heavy-metal containing QDs in the same applications. ${ }^{41}$

$\mathrm{Si}$ is an indirect bandgap semiconductor with an $\mathrm{E}_{\mathrm{g}}$ of $1.1 \mathrm{eV}$. Even though radiative recombination of an electron-hole pair across the indirect bandgap is momentum forbidden in the bulk material, Si nanomaterials exhibit strong, visible PL. This can be attributed to the Heisenberg uncertainty principle, by which it can be rationalized that as the particle volume decreases, the uncertainty in the position of the charge carriers decreases, which results in an increase in the uncertainty in their momentum. ${ }^{11}$ Thus, as the size of the crystalline domains are reduced towards the Bohr exciton radius of $\mathrm{Si}\left(4.3 \mathrm{~nm}^{49}\right)$, the selection rule thus becomes relaxed and radiative recombination becomes less forbidden.

According to theoretical predictions, in an ideal Si NP the optical absorption and emission pathways are interband transitions, and the size of the Si NP is the dominant variable in determining the energy of the transitions. ${ }^{6,50}$ The effective mass approximation (EMA) equation is commonly used to predict the $\mathrm{E}_{\mathrm{g}}$ for a given particle diameter. ${ }^{6,9}$ The salient feature of each of these equations is the dependence of $\mathrm{E}_{\mathrm{g}}$ on the inverse square of the nanoparticle diameter. Equation 1 is one version of the EMA, which 
was adapted by Hessel et al., ${ }^{9}$ that summarizes the relationship between particle diameter $(\mathrm{d})$ and $\mathrm{E}_{\mathrm{g}}$ :

$$
E(d)=E_{g}+\frac{h^{2} \pi^{2}}{2 d^{2}}\left(\frac{1}{m_{e}^{*}}+\frac{1}{m_{h}^{*}}\right)-\frac{1.786 e^{2}}{\varepsilon_{R} d} \quad \text { Equation } 1
$$

where $E(d)$ is the bandgap energy for a given Si NP diameter, $m_{e}$ and $m_{h}$ are the effective masses of an electron and a hole, $e$ is the electron charge, and $\varepsilon_{\mathrm{R}}$ is the relative permittivity of $\mathrm{Si}$ (11.86). In this equation, the first term, $E_{g}$, is the bandgap of bulk $\mathrm{Si}$ $(1.1 \mathrm{eV})$, the second term in the equation corresponds to the energy associated with a quantum well, and the final term accounts for electron-hole attraction and is called the Coulomb term. The relationship described in Equation 1 is plotted in Figure 1.4 as the bandgap energy of the confined Si NP domain versus the Si NP diameter (solid line). ${ }^{9}$

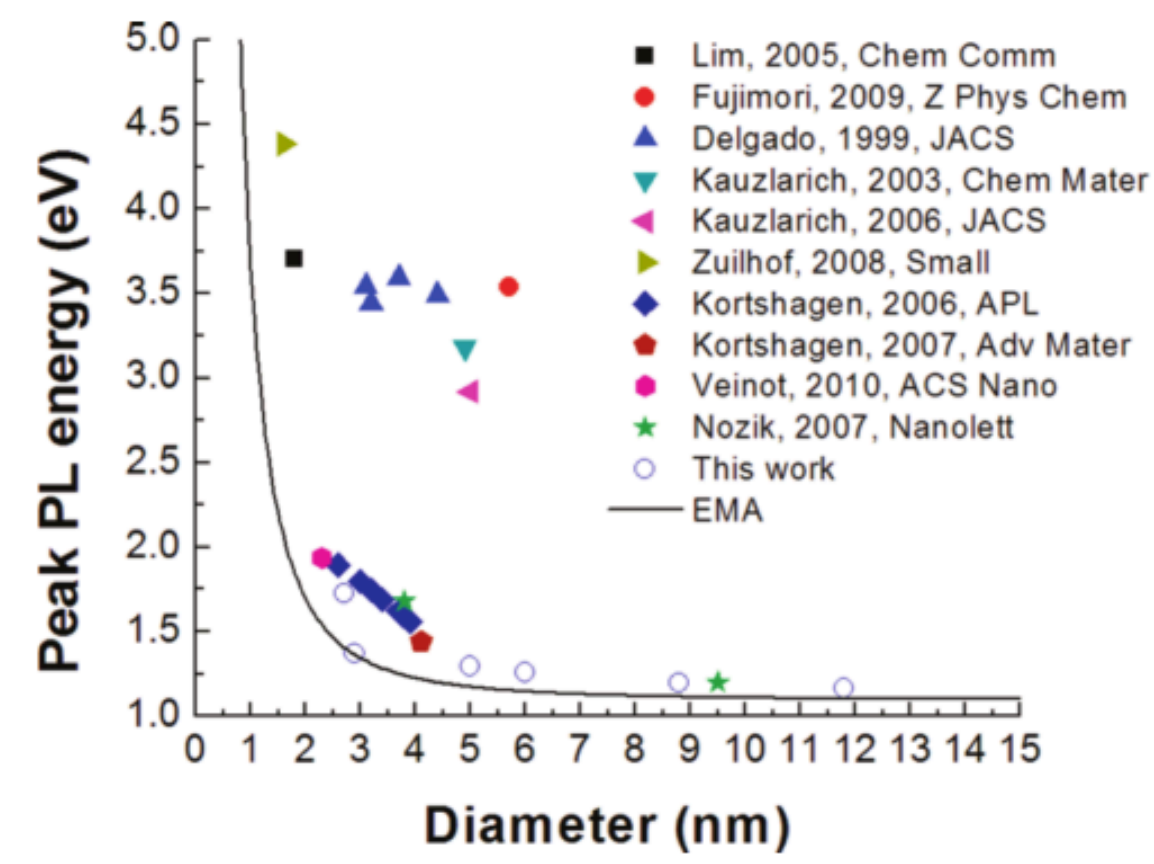

Figure 1.4. Plot of peak PL energy versus Si NP diameter. EMA prediction shown as a solid line. Symbols represent experimental results reported in literature. Reprinted with permission from (Chem. Mater. 2012, 24, 393-401). Copyright (C 2012, American Chemical Society. 
According to Figure 1.4, the average Si NP diameter would theoretically need to be between approximately $1.5-2 \mathrm{~nm}$ in order for visible PL to be observed, although experimentally, visible PL has frequently been observed from Si NP samples with larger population mean diameters. It should be noted that the EMA has been reported to overestimate the effect of QC on the bandgap energy. ${ }^{6}$ Even so, the EMA is commonly used in the literature to predict the size-dependent bandgap energies expected for Si NPs. ${ }^{6,7,9,51}$

\subsection{Observed photoluminescence from nanocrystalline silicon}

\subsubsection{Size-dependent photoluminescence from nanocrystalline silicon}

An ideal Si NP has a large number of Si atoms located on the edges, corners, and faces of the nanocrystal that have less than four bonds to other Si atoms. Consequently, the surface Si coordination environments are not complete, which can be expected to result in high energy surface defects, such as free radicals (called dangling bonds) or $\mathrm{Si}=\mathrm{Si}$ dimers, filling the remaining sites. $[\mathrm{REF}]$ Structures such as $\mathrm{Si}=\mathrm{Si}$ dimers and free radicals are unstable, and so these sites can react with either atomic or molecular passivants, such as hydride or hydrogen, to result in a lower energy surface.

Hydride-passivation of the reactive surface $\mathrm{Si}$ sites is frequently used in computational modeling of ideal systems, owing to the negligible effect hydrogen has on the Si NP electronic structure as well as its ability to provide adequate coverage of terminal Si bonds. ${ }^{49,52-55}$ Experimentally, evidence for PL due solely to QC has been shown by numerous groups for hydride-passivated nanocrystalline silicon. For example, in a study published by Wolkin et al. in 1998, they reported that when Si NPs are 
hydride-passivated, the predominant exciton recombination pathway is an interband transition. ${ }^{52}$ Several other groups have also shown PL dependence on NP size for initially large, red-emitting, hydride-passivated Si NPs (H-Si NPs) as their sizes are chemically modified. ${ }^{9,21,30,56}$ In these studies, decreases in the Si NP diameter are concurrent with increased PL energies, which supports QC theory. ${ }^{9,21,30,52,56}$ However, even though QCbased PL has been reported for $\mathrm{H}-\mathrm{Si}$ NPs, the $\mathrm{Si}-\mathrm{H}$ bond is known to be thermodynamically weak relative to other $\mathrm{Si}-\mathrm{X}$ bonds $(\mathrm{X}=\mathrm{O}, \mathrm{N}, \mathrm{F}$, etc. $)$ and therefore it is expected to be unstable in experimental and ambient conditions. As a result, the H-Si NP surface is expected to be highly reactive and prone to formation of surface defects that could impact the interband PL.

\subsubsection{Effect of defects on photoluminescence from silicon nanoparticles}

It is generally understood that the surface composition of Si NPs heavily influences the type and number of carrier recombination pathways present within a specific Si NP. ${ }^{40,43-53}$ For example, surface reconstructions, chemical impurities, lattice vacancies, dislocations, etc., can produce characteristic energy states (defect states) within the electronic structure of the Si NP with a corresponding DoS proportional to their concentration within the crystal lattice. ${ }^{5}$ However, for a defect state to be electronically active and interfere with interband transitions, its associated energy level or band of levels must lie within the silicon bandgap. ${ }^{5}$ Since the average time it takes for an electron or hole to relax to a defect state is up to four orders of magnitude faster than interband recombination for silicon, photogenerated carriers are highly susceptible to capture by electronically-active defect states. ${ }^{5}$ As a result, if defect states are present and 
electronically active, they are expected to dominate the observed photophysical properties.

Most chemical modifications made to the H-Si NP surface, with the exception of alkyl-passivation, ${ }^{9}$ are reported to introduce electronically active defect states into the electronic structure that can reduce the radiative yield of interband transitions. For example, oxidation of high surface area $\mathrm{Si}$ occurs readily in ambient and laboratory conditions and is frequently cited to coincide with sudden, drastic changes in the observed PL. ${ }^{23,51,52,55,65-67}$ However, the observed increase or decrease in emission energy that oxygen passivation causes varies from study to study.

Several groups have studied the effects of oxide shells on the photophysical properties of nanocrystalline $\mathrm{Si}^{56,60,61}$ In one study, ${ }^{69} 720 \mathrm{~nm}$ PL $\left(\lambda_{\text {exc }}=488 \mathrm{~nm}\right)$ from p-Si was attributed to non-bridging oxygen hole (NBOH) centers (e.g., Si-O- and Si-O---H---O-Si) present at the interface of the nanocrystalline $\mathrm{Si}$ domains and the surface oxide. These NBOH centers have the same chemical composition as the NBOH centers that have been reported in a separate study to be the cause of $652 \mathrm{~nm} \mathrm{PL}$ $\left(\lambda_{\text {exc }}=514 \mathrm{~nm}, 258 \mathrm{~nm}\right)$ from a-SiO 2 , which lacks elemental Si domains. ${ }^{68}$

Changes in the observed PL from nanocrystalline Si have also been attributed to changes in the surface oxidation of nanocrystalline Si domains. For example, in two studies conducted by Kanemitsu and coworkers, ${ }^{65,70}$ the group provided evidence for carrier trapping by electronic states resulting from defects at the interface between Si NPs and an $\mathrm{a}_{-} \mathrm{SiO}_{2}$ surface layer. In the latter study, originally non-photoluminescent Si NPs were observed to emit intense $750 \mathrm{~nm}$ PL $\left(\lambda_{\mathrm{exc}}=355 \mathrm{~nm}\right)$ following the formation of an a-SiO ${ }_{2}$ surface layer. ${ }^{61}$ Conversely, others have reported the development of high energy 
PL from originally red-emitting Si NPs concurrent with surface oxidation. ${ }^{63,67}$ For example, in a study conducted by our group, we characterized changes in the photophysical properties of initially $690 \mathrm{~nm}$-emitting $\left(\mathrm{d}=4 \mathrm{~nm}, \lambda_{\text {exc }}=370 \mathrm{~nm}\right)$, alkylpassivated Si NPs following exposure to a series of $n$-alcohols varying in hydrocarbon chain length. ${ }^{67}$ We reported that the development of surface oxide (observed by FT-IR) was associated with the development of a new emission band with a $\lambda_{\max }$ of $450 \mathrm{~nm}$ when excited at $370 \mathrm{~nm}$. This was observed to occur to the greatest extent when the Si NPs were exposed to the shortest chain alcohols that were least sterically hindered from access to the Si NP surfaces. On the basis of all data, including size analysis by TEM and time-resolved PL spectroscopy, we proposed that the developing high-energy PL originated from the formation of an oxide-related extrinsic state. However, the exact chemical identity of the extrinsic, emissive center remains disputed.

Carrier trapping by defect states can also lead to the emission of a phonon, rather than a photon (i.e., can occur non-radiatively). For Si NPs, the most commonly suspected source of non-radiative decay is the Si-based radical trap state (also called a dangling bond). ${ }^{13,49,57,65,67,71-73}$ In fact, it has been suggested that dangling bonds form highly efficient non-radiative recombination centers, such that the presence of a single dangling bond in a Si nanocrystal is sufficient to prevent PL. ${ }^{49}$ Experimentally, dangling bonds have been shown to be present both on the surface of silicon nanoparticles, ${ }^{74}$ as well as at the crystal-oxide interface of oxidized Si NPs. ${ }^{56,72}$ In these studies, the presence of Sibased radicals was correlated with reduced PL intensities, which is in good agreement with theoretical predictions. 
In a computational study conducted by Delerue and co-workers, the influence of dangling bonds on the electronic structure of porous silicon (p-Si) was analyzed. ${ }^{49}$ In the study, the authors analyzed the mechanism of charge carrier trapping at an unoccupied trap state that was located just below the conduction band minimum in energy. They reported that for nanocrystalline silicon with bandgaps less than $2.2 \mathrm{eV}(560 \mathrm{~nm})$, nonradiative recombination of an exciton at a neutral dangling bond can occur quickly $\left(\right.$ rate $\left.=\sim 10^{5}-10^{7} \mathrm{~ms}^{-1}\right)$ via a two step process in which the electron and hole are trapped sequentially. The study also reported that non-radiative decay could happen so quickly that the presence of a single dangling bond on the surface of p-Si would likely be sufficient to prevent interband carrier recombination.

\subsection{Surface defect prevention in silicon nanoparticles}

In order to prevent the development of defect states, it is necessary to passivate the surface without the formation of surface species that have electronic states that lie within the bandgap of the Si NP. Hypothetically, a high quality amorphous silicon dioxide $\left(\mathrm{a}-\mathrm{SiO}_{2}\right)$ layer could passivate the surface without forming electronically active defect states, also called trap states. One argument in favor of using a- $\mathrm{SiO}_{2}$ as a surface passivant is that it can in theory passivate the surface without disrupting the silicon lattice, where disruptions or distortions of the lattice could result in trap states. ${ }^{5,11}$ Second, the band edges of the $\mathrm{a}-\mathrm{SiO}_{2}$ layer lie outside the bandgap of silicon $\left(\mathrm{E}_{\mathrm{g}}=\sim 8.9 \mathrm{eV}\right)^{75}$. Thus, $\mathrm{a}-\mathrm{SiO}_{2}$ is considered electronically inactive and is considered to be an electrical insulator. However, the formation of such a layer is difficult to achieve experimentally, 
and surface oxidation typically results in the formation of electronically active states within the bandgap. ${ }^{23,51,52,55,63,65-68,70}$

Alkyl-passivation has long been used in order to surface stabilize bulk and nanocrystalline silicon surfaces, in particular against ambient oxidation. Alkylpassivation is thought to have negligible effects on the photophysical properties of the Si domains, similar to hydride-passivation. ${ }^{76}$ To this end, experimental evidence suggests that the Si NP surface can undergo alkyl-passivation as a means to preserve interband PL. 9 Consequently, numerous methods have been developed to form stable, Si-C passivated surfaces. The first report of covalently bound alkyl monolayers on planar silicon surfaces was achieved by the pyrolysis of diacyl peroxides in the presence of hydride-passivated surfaces. ${ }^{77}$ The authors postulated that the reaction proceeded by initial abstraction of surface bound hydrides, followed by passivation of the intermediate silicon radical species by a second equivalent of alkyl radical generated by the pyrolysis reaction. While the reaction produced alkyl passivated surfaces, as evidenced by complete loss of the initial $\mathrm{Si}-\mathrm{H}$ bonding features, as much as $30 \%$ of the monolayer formed was shown to be $\mathrm{Si}-\mathrm{O}-\mathrm{C}$ passivation as opposed to pure $\mathrm{Si}-\mathrm{C}$ bonding.

Since then, the majority of the reactions that have been reported to yield alkylpassivated Si surfaces have focused on the addition of $\mathrm{Si}-\mathrm{H}$ bonds across unsaturated hydrocarbon bonds (i.e., hydrosilylation), ${ }^{78}$ as shown in Scheme 1.1. The two most

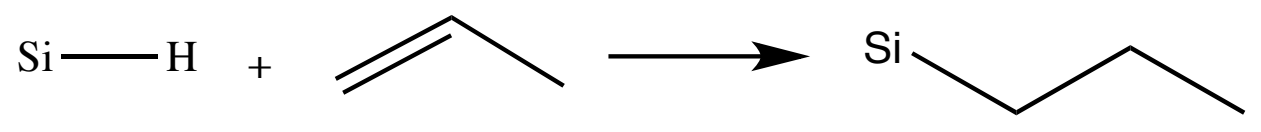

Scheme 1.1. Hydrosilylation reaction resulting in formation of $\mathrm{Si}-\mathrm{C}$ bonds. 
common hydrosilylation techniques in the literature are organometallic catalysis and radical initiated reactions, the latter of which is the subject of discussion in Chapter 3.

Lewis acid catalyzed hydrosilylation was among the first organometallic catalysis reactions that was shown to form stable, Si-C bound alkyl monolayers on $\mathrm{Si}$ surfaces. ${ }^{76,79,80}$ For example, reaction of hydride-passivated porous silicon surfaces with terminal alkenes and alkynes in the presence of catalytic amounts of $\mathrm{EtAlCl}_{2}$ was shown to form stable Si-C bonding at room temperature. ${ }^{80}$ In that study, the authors reported a maximum reaction efficiency of $28 \%$ when hydrosilylation was conducted with terminal alkenes, which was determined by calculating the percent decrease in the integrated intensity of the $\mathrm{Si}-\mathrm{H}$ bond at $2100 \mathrm{~cm}^{-1}$, as monitored by infrared (IR) spectroscopy. They furthermore showed that the reaction afforded high tolerance to $\omega$-functionalized 1-alkenes, with a strong preference for alkyl-passivation of the surface.

The majority of hydrosilylation reactions reported to date fall into the class of radical initiated reactions, which have been shown to produce surfaces with much higher alkyl coverage than their organometallic catalysis counterparts. Thermal- and UVassisted hydrosilylation reactions are perhaps the most commonly cited silicon surface alkylation reactions. ${ }^{81-88}$ These reactions have long been accepted by most (not all ${ }^{83}$ ) groups to proceed via the addition of surface silicon radicals to unsaturated carboncarbon bonds. Furthermore, these reactions are commonly accepted to be initiated by the homolytic cleavage of the $\mathrm{Si}-\mathrm{H}$ bond, which is accomplished either thermally or photolytically and results in reactive silicon radicals. It should be noted that a recent report suggested that homolytic cleavage of surface $\mathrm{Si}-\mathrm{Si}$ bonds, rather than $\mathrm{Si}-\mathrm{H}$ bonds, 


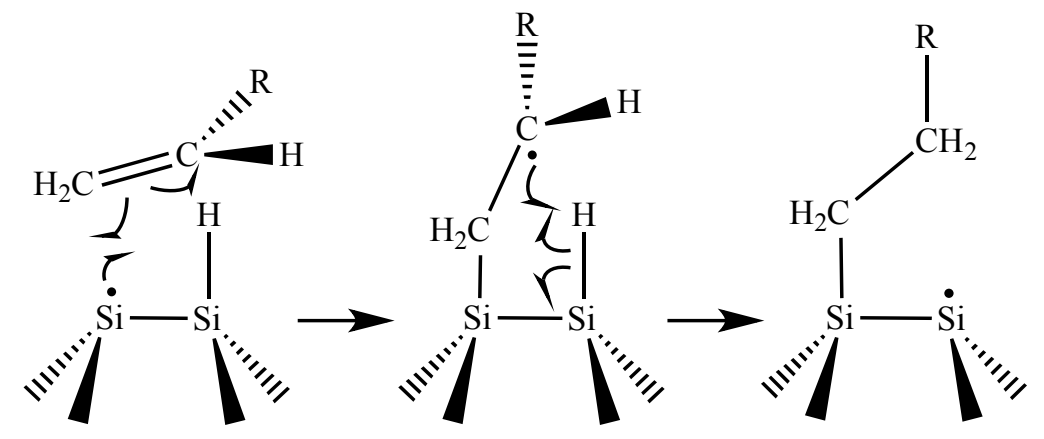

Scheme 1.2. Reaction of a Si surface radical with an alkene.

is the source of silicon radicals, although either way, the result of the reaction is alkylpassivated surfaces. $^{82}$

In radical-mediated hydrosilylation, it is generally accepted that Si radicals react with terminal unsaturated carbon bonds at the $\mathrm{C} 1$-position, forming an intermediate alkyl radical at the C2-position. Subsequently, propagation of the surface passivation continues by abstraction of a neighboring hydride from the Si surface by the alkyl radical, thereby forming a new reactive Si radical site, as shown in Scheme 1.2. Thermal hydrosilylation reactions using dry, degassed 1-alkenes have been reported to produce surfaces with up to $70 \%$ alkyl passivation, depending on the steric hindrance of the ligand at the surface. ${ }^{83-86}$ $\mathrm{Si}-\mathrm{H}$ bond cleavage, and subsequent hydrosilylation of the $\mathrm{Si}$ surface, has also been reported to have been accomplished by UV irradiation $(\lambda \leq 350 \mathrm{~nm})$ at room temperature, producing surfaces with as high as $45 \%$ surface coverage. ${ }^{87}$

\subsection{Motivation for the following studies}

To the author's knowledge, there are no reports of hydrosilylation resulting in a Si NP surface with greater than $70 \%$ alkyl-coverage. This means that the surface composition of alkyl-passivated Si NPs, which has been well-established as having a 
large role in the optical activity of Si NPs, is not homogenous. Furthermore, un-reacted Si-H surface sites may remain immediately following hydrosilylation. This may lead to surface oxidation over time, since the $\mathrm{Si}-\mathrm{H}$ bond has been well-established as an unstable surface bond. Thus, the further reactivity of $\mathrm{Si}-\mathrm{H}$ bonds remaining after hydrosilylation could result in unstable PL over time.

Ideally, by obtaining a better understanding of the nature and reactivity of the defects present on the H-Si NP surface, one could envision being able to mitigate the reactivity of the Si NP surface and, as a result, optimize the optical performance of the material. It is therefore prudent to examine a simple system, such as H-Si NPs, to understand the conditions under which specific defects form, in addition to how these defects impact light absorption and emission by Si NPs.

Thus, this dissertation examines the correlation between changes in the surface composition of H-Si NPs and changes in PL, as a function of chemical environment and time. Using spectroscopy to examine the chemical and photophysical changes to the Si NPs, we are able to correlate changes in the optical properties due to the formation of surface defects with the conditions of their formation. These results shed light on the expected behaviors of Si NPs under conditions typically present in the applications outlined in Section 1.3, and provide guidance as to how deleterious defects can be avoided to improve the optical property stability of Si NPs. 
Chapter 2

Synthesis of hydride-passivated silicon nanoparticles

from polymeric $\mathrm{HSiO}_{1.5}$

\section{$2.1 \quad$ Introduction}

A thorough description for the synthesis of red-emitting, hydride-passivated silicon nanoparticles (H-Si NPs) is discussed below. First, $\mathrm{HSiCl}_{3}$ undergoes hydrolysis and polycondensation to produce a polymer with a putative stoichiometry of $\left(\mathrm{HSiO}_{1.5}\right)_{\mathrm{n}}$. Then, nanocrystalline $\mathrm{Si}^{0}$ domains encased in an amorphous silicon oxide matrix are produced through a high temperature processing of the polymer. Finally, H-Si NPs are isolated from the oxide by a wet chemical etch. Chemical characterizations are performed after each reaction step en route to the H-Si NP suspensions that are further studied in Chapters 2 through 5 of this dissertation.

\subsection{Experimental}

\subsubsection{Materials}

Trichlorosilane $\left(\mathrm{HSiCl}_{3}, 98 \%\right.$, Ar-protected, Alfa Aesar, Ward Hill, MA), hydrofluoric acid (HF, ACS reagent, 48-51\% solution in water, Acros Organics, NJ), ethanol (abs-EtOH, $\mathrm{C}_{2} \mathrm{H}_{5} \mathrm{OH}$, anhydrous, Pharmco-AAPER, Brookfield, CT), $n$-hexane $\left(\mathrm{C}_{6} \mathrm{H}_{14}\right.$, Sigma-Aldrich, St. Louis, $\left.\mathrm{MO}\right)$, and toluene (reagent grade) were purchased and used without further purification. Millipore water $\left(\mathrm{H}_{2} \mathrm{O}, 18 \mathrm{M} \Omega \bullet \mathrm{cm}\right.$ resistivity) was used exclusively in the polymerization and etching procedures. Laboratory deionized water (DI- $\left.\mathrm{H}_{2} \mathrm{O}\right)$, methanol $\left(\mathrm{MeOH}, \mathrm{CH}_{3} \mathrm{OH}\right.$, reagent grade), and nitric acid $\left(\mathrm{HNO}_{3}\right.$, reagent 
grade, Fisher Scientific, MA) were used to clean the aluminum disks for elemental analysis.

\subsubsection{Synthesis of polymeric $\mathrm{HSiO}_{1.5}$}

A $100 \mathrm{~mL}$ 2-neck round bottom flask was equipped with a $1 \mathrm{~cm}$ egg-shaped magnetic stir bar and sealed with a line valve and a rubber septum. The flask then underwent three cycles of evacuation followed by purging with Ar to eliminate ambient air from the system. Throughout the remainder of the procedure, the flask was left under an over pressure of Ar. Meanwhile, the flask was brought to $0^{\circ} \mathrm{C}$ by submerging the entirety of the flask in an ice bath (all but the flask's necks were below the water level). Then, $5.0 \mathrm{~mL}$ of $\mathrm{HSiCl}_{3}$ was injected rapidly into the flask using a disposable syringe and needle. CAUTION: $\mathrm{HSiCl}_{3}$ is extremely volatile, and will react with moisture in the air. It should remain under a blanket of Ar at all times, and its exposure to ambient temperature should be be kept to a minimum (stored at $4{ }^{\circ} \mathrm{C}$ in dark). Always use syringes with Luer locks when handling $\mathrm{HSiCl}_{3}$ to prevent accidental needle ejection.

The $\mathrm{HSiCl}_{3}$ was equilibrated at $0^{\circ} \mathrm{C}$ over the course of $2-5 \mathrm{~min}$ following injection, at which point $5.5 \mathrm{~mL}$ of $\mathrm{H}_{2} \mathrm{O}$ was rapidly injected into the flask using a new disposable syringe and needle. As the $\mathrm{H}_{2} \mathrm{O}$ was injected, the formation of a white polymer was observed where the $\mathrm{H}_{2} \mathrm{O}$ came into contact with the $\mathrm{HSiCl}_{3}$. If the $\mathrm{H}_{2} \mathrm{O}$ is injected too slowly, volitalized $\mathrm{HSiCl}_{3}$ can react with $\mathrm{H}_{2} \mathrm{O}$ inside the needle and clog it. To maximize the contact between the $\mathrm{H}_{2} \mathrm{O}$ and the $\mathrm{HSiCl}_{3}$, the flask can be tilted or the stream of water can be "moved around". CAUTION: this reaction is highly exothermic, so maintaining the entire round bottom portion of the flask below the ice water level is important. It should also be noted that $\mathrm{HCl}_{(g)}$ is a byproduct of the reaction, and it is 
important to keep the flask open to the Schlenk line otherwise there is risk of explosion, or the septum being pushed off, due to rapid volume increase.

Following injection of the $\mathrm{H}_{2} \mathrm{O}$, the reaction was left to react for $5-10$ min at $0^{\circ} \mathrm{C}$. Afterward, the reaction was vented using a disposable needle under flowing Ar to purge the $\mathrm{HCl}_{(\mathrm{g})}$ from the flask. An acid trap was fashioned to minimize the corrosion of metallic objects in the fume hood. The $\mathrm{pH}$ of the air flowing out of the vent was tested periodically with wet litmus paper for $2-5 \mathrm{~h}$ to monitor the loss of $\mathrm{HCl}$. When the $\mathrm{pH}$ no longer tested acidic, the polymer was then dried under flowing Ar for $24 \mathrm{~h}$. A typical synthesis yields approximately $1.25 \mathrm{~g}$ of dry polymer. The chemical composition of the polymer was analyzed by infrared (IR) and X-ray photoelectron (XP) spectroscopies. Polymer samples were stored in a vials under a blanket of Ar until further use.

\subsubsection{Thermal processing of polymeric $\mathrm{HSiO}_{1.5}$}

The dry polymer was annealed at high temperatures under flowing $\mathrm{N}_{2}$ to produce Si NPs embedded in a matrix of $\mathrm{SiO}_{2}$. In each experiment, approximately $0.5 \mathrm{~g}$ of $\left(\mathrm{HSiO}_{1.5}\right)_{\mathrm{n}}$ was loaded into a $5.0 \mathrm{~mL}$ alumina combustion boat (Coors). The sample was then placed into a quartz tube (13/16" ID, 15/16" OD) positioned within a horizontal tube furnace (Lindberg/Blue M, Thermo Fisher Scientific, USA). The temperature of the furnace was increased at a rate of $20^{\circ} \mathrm{C} / \mathrm{min}$ to a maximum temperature between 950 and $1100^{\circ} \mathrm{C}\left(50^{\circ} \mathrm{C}\right.$ intervals). The furnace was then held at that temperature for a total time between $30 \mathrm{~min}$ and $10 \mathrm{~h}$ to elicit varying degrees of thermal disproportionation of the suboxide into $c$-Si and $a-\mathrm{SiO}_{2}$. The temperature of the furnace was then brought back down to room temperature over the course of $3-4 \mathrm{~h}$. In some cases, the cooling setting 
of a heat gun was used to increase the rate of cooling when the oven temperature fell below $200^{\circ} \mathrm{C}$.

After annealing, the resulting coarse, light brown powder was then ground into a finer powder either using a mortar and pestle, or by mechanical milling inside a tungsten carbide (or stainless steel) lined milling vial with two $1 \mathrm{~cm}$ tungsten carbide (or stainless steel) balls. Mechanical milling was done for $10 \mathrm{~s}$ (time established by prior student) using a Spex 8000M mill mixer (SPEX Sample Prep, Metuchen, NJ). XRD and XPS were conducted on select finely ground powders to study the crystallinity and chemical composition, respectively.

\subsubsection{Liberation of Si NPs from oxide matrix}

After grinding to a fine powder, the annealed powder was next chemically etched to isolate the Si domains as hydride-passivated Si NPs (H-Si NPs). First, approximately $0.5 \mathrm{~g}$ of the finely ground, annealed powder was added to a $150 \mathrm{~mL}$ Teflon sample cup. Next, $5.0 \mathrm{~mL}$ of abs-EtOH and $5.0 \mathrm{~mL}$ of $\mathrm{H}_{2} \mathrm{O}$ were added, and the sample was sonicated to disperse the solid and homogenize the mixture. A $2 \mathrm{~cm}$ magnetic stir bar was then added to the sample cup, the mixture was set to stir, and $5.0 \mathrm{~mL}$ of HF was added. The addition of HF to the mixture resulted in the visible evolution of a gas. The mixture was loosely covered with a plastic weigh boat and left to stir for $60 \mathrm{~min}$ in ambient air and light. CAUTION: HF is a weak acid with a strong affinity for $\mathrm{Ca}^{2+}$. It will react in stoichiometric equivalents with calcium in bones; it is only to be used with extreme caution. All reactions involving $H F$ should be done in a hood designated for HF use. Always alert others in the surrounding area when it is being handled, and keep 
Calgonate cream and calcium salts nearby in the case of accidental skin contact or spillage.

After etching for $60 \mathrm{~min}$, the aqueous mixture was carefully poured into a $50 \mathrm{~mL}$ polypropylene centrifuge tube, and $\sim 15.0 \mathrm{~mL}$ of either hexane or toluene was added on top of the solution. The tube was then tightly capped and mixed using a vortex mixer to phase-transfer the H-Si NPs into the non-polar phase. The suspension was then left to settle for $\sim 10 \mathrm{~s}$ before $5.0 \mathrm{~mL}$ of the suspension containing H-Si NPs was extracted from the middle of the non-polar layer using a $5 \mathrm{~mL}$ pipettor and added to a storage vessel (vial, round bottom, etc.). An additional $5.0 \mathrm{~mL}$ of either hexane or toluene was then added to the centrifuge tube, the tube sealed and the solution was mixed. Another $5.0 \mathrm{~mL}$ of the suspension was then extracted from the middle of the non-polar layer and added to the secondary storage vessel. This extraction was performed a total of $8-10$ times resulting in a total of $40-50 \mathrm{~mL}$ of suspended H-Si NPs. The H-Si NPs were then analyzed by IR, PL, and XP spectroscopies as well as by transmission electron microscopy (TEM). The suspensions were stored under Ar until further use.

\subsubsection{Fourier-transform infrared (FT-IR) spectroscopy}

FT-IR spectroscopy was conducted using a Thermo Nicolet iS10 spectrophotometer equipped with a single-bounce diamond attenuated total reflectance (ATR) attachment. Suspensions were measured by depositing drop-cast films onto the ATR attachment and allowing the solvent to evaporate at room temperature. Solid samples were directly deposited onto the diamond crystal with a disposable plastic spatula and compressed onto the crystal. For each sample, a total of 64 scans were collected from $4000-650 \mathrm{~cm}^{-1}\left(2 \mathrm{~cm}^{-1}\right.$ resolution $)$ and were signal averaged to yield the 
final IR spectrum. In some cases, a linear baseline subtraction was required to correct for light scattering intensity, which increases linearly with energy and results in an apparent decrease in transmittance at higher energies; no other data processing was conducted.

\subsubsection{X-ray photoelectron spectroscopy (XPS)}

Polymer samples before and after annealing were prepared for XPS by suspending a small amount (end of a spatula tip) of the solids in a minimal volume of hexane to create slurries. The slurries were then drop-cast onto $\mathrm{Al}$ disks and dried at room temperature. The $\mathrm{Al}$ disks were washed in $\mathrm{HNO}_{3}$ and rinsed with $\mathrm{DI}-\mathrm{H}_{2} \mathrm{O}$ and $\mathrm{MeOH}$ thoroughly prior to sample preparation. The Al substrate was then secured onto an aluminum sample platen with commercial double-sided tape.

XPS was conducted using a Versaprobe II (Physical Electronics Inc., PHI, Chanhassen, MN) operating at $15 \mathrm{kV}$ and $25 \mathrm{~W}$, with a monochromatic $\mathrm{Al} \mathrm{K} \alpha \mathrm{X}$-ray beam $(100 \mu \mathrm{m}$ spot size $)$. Data were collected at a rate of $20 \mathrm{~ms} / \mathrm{step}$. Survey scans were collected with a pass energy of $187.5 \mathrm{eV}$ and step size of $0.2 \mathrm{eV}$ ( 2 scans averaged). All spectra were collected with dual neutralizers enabled. Detailed scans of specific elemental binding energies were collected with a pass energy of $23.5 \mathrm{eV}$ and a step size of $0.1 \mathrm{eV}$ for better peak resolution. A total of 6 scans of the $\mathrm{Si}(2 \mathrm{p})$ and $\mathrm{O}(1 \mathrm{~s})$ regions were averaged to yield the final spectra, while 3 scans of the other regions were averaged.

Peak fitting of the measured XP spectra was done using the associated XPS Multipak software suite. Spectra were calibrated against the adventitious $C(1 \mathrm{~s})$ peak (284.9 eV), and smoothing and background subtraction were applied using the default software settings. Integrated peak areas were used to determine the relative concentrations of elements and atomic oxidation states. 


\subsubsection{X-ray diffraction $(X R D)$}

XRD was performed on a Rigaku Ultima IV X-ray diffraction system (Rigaku Americas, The Woodlands, Texas) with a monochromated $\mathrm{Cu} \mathrm{K \alpha} \mathrm{X}$-ray beam operating in Bragg-Brentano geometry. Data were collected between $10^{\circ}$ and $60^{\circ}$ 2-theta in $0.1^{\circ}$ intervals at a rate of $3 \% / \mathrm{min}$ on finely ground powders.

Peak fitting of the measured data was done with the associated PDXL software according to the standardized procedure outlined in the user manual (Version 1.8.0.3) in order to resolve the overlapping c-Si and a-SiO 2 phases. Briefly, the relative heights and widths of the overlapping peaks were manually adjusted in tandem with the background in order to minimize the difference between the calculated pattern and the measured data. Scherrer analysis of the c-Si phase line broadening was used to elucidate qualitative trends in the effects of thermal processing conditions on c-Si domain size. Furthermore, the percent crystallinity of the annealed samples was estimated by comparison of the integrated peak area of the c-Si phase to the overall integrated area of all phases.

\subsubsection{Photoluminescence (PL) spectroscopy}

PL spectroscopy measurements of H-Si NP suspensions were performed with a Shimadzu 5301-RFPM spectrophotometer equipped with a Xe arc-laser source. Samples were excited with monochromatic $360 \mathrm{~nm}$ light through a $3 \mathrm{~nm}$ excitation slit, and spectra were recorded from $390-700 \mathrm{~nm}$ after passing through a $5 \mathrm{~nm}$ emission slit. Photoluminescence excitation (PLE) spectra were collected by varying the energy of the excitation beam from 230 to $\left(\lambda_{\max }-10\right) \mathrm{nm}$ while monitoring the intensity of the $\lambda_{\max }$ determined from the PL spectrum. 


\subsubsection{Transmission electron microscopy (TEM)}

A Technai-F20 transmission electron microscope (FEI, Hillsboro, OR) operating at $200 \mathrm{keV}$ and an extraction voltage of $4500 \mathrm{~V}$ was used to image the Si NPs. Samples were prepared either by drop-casting Si NP suspensions directly onto a holey-carbon coated $3 \mathrm{~mm} \mathrm{Cu}$ grid or by dipping a grid into the suspension immediately following sonication of the suspension to maximize dispersion of the Si NPs.

\subsection{Results and discussion}

\subsubsection{Synthesis of polymeric $\mathrm{HSiO}_{1.5}$ from $\mathrm{HSiCl}_{3}$}

$\left(\mathrm{HSiO}_{1.5}\right)_{\mathrm{n}}$ was synthesized from $\mathrm{HSiCl}_{3}$ according to the hydrolysis and polycondensation reaction shown in Scheme 2.1. In the first step, Si-OH bonds are formed by the hydrolysis of the $\mathrm{Si}-\mathrm{Cl}$ bonds in $\mathrm{HSiCl}_{3}$, forming $\mathrm{HCl}$ gas as a byproduct. The Si-OH bonds then undergo polycondensation, which forms a polymeric, Si-O-Si bonded network, where each of the three oxygen $(\mathrm{O})$ atoms bound to a single $\mathrm{Si}$ atom are shared between two units. As can be seen in Figure 2.1, the IR spectrum of the dried polymer contains overlapping peaks at $1050 \mathrm{~cm}^{-1}$ and $1090 \mathrm{~cm}^{-1}$, both of which are in the region that has been attributed to Si-O bonding. ${ }^{9,34,40,47,58,63,69,72,75-83}$ Evidence for the preservation of Si-H bonding is also shown by the peak at $2240 \mathrm{~cm}^{-1}$ in the IR spectrum.

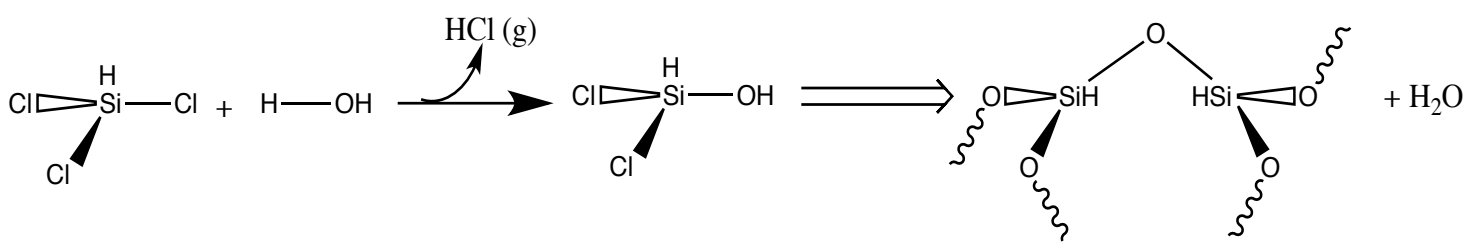

Scheme 2.1. Mechanism for the hydrolysis and polycondensation of $\mathrm{HSiCl}_{3}$. 


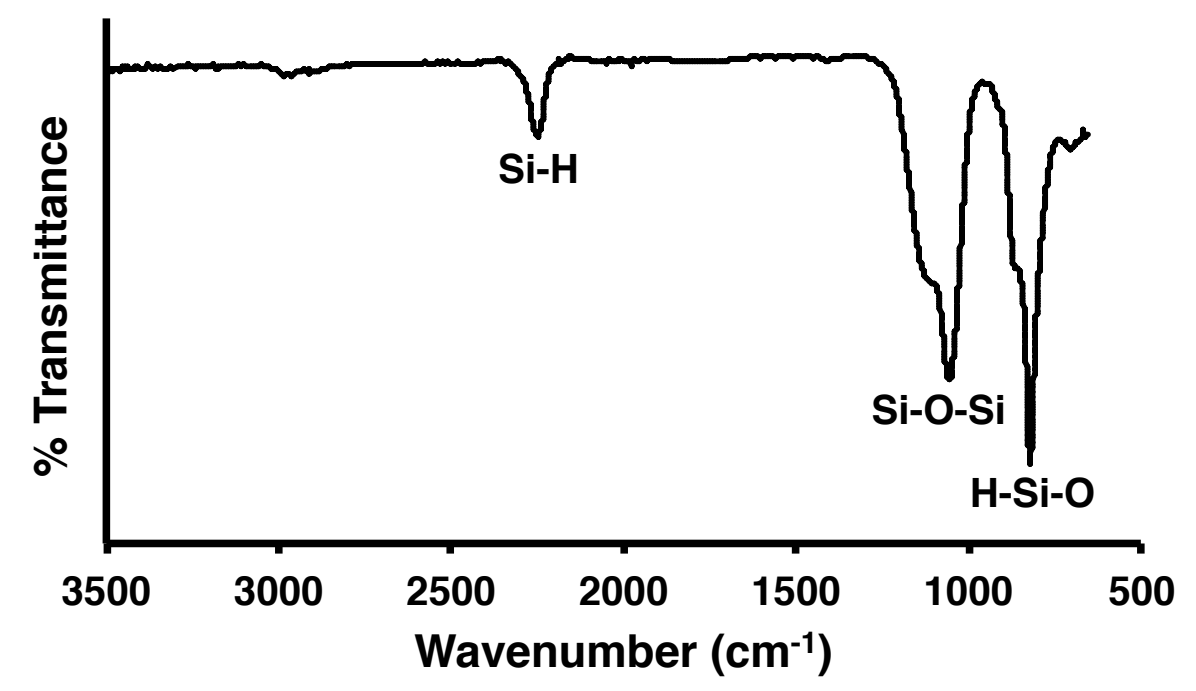

Figure 2.1. FT-IR spectrum of dry $\left(\mathrm{HSiO}_{1.5}\right)_{\mathrm{n}}$.

This peak is typically assigned as an $\mathrm{Si}-\mathrm{H}$ stretch, where the $\mathrm{Si}$ atom is also bound to an $\mathrm{O}$ atom. ${ }^{94}$ Finally, the peak at $820 \mathrm{~cm}^{-1}$ corresponds to H-Si-O vibrational modes. ${ }^{21}$

Compositional analysis of the dry polymer was conducted by XPS. A survey spectrum ranging from $1100-0 \mathrm{eV}$ is shown in Figure 2.2. The two leftmost arrows in the figure correspond to the energies where characteristic $\mathrm{Cl}$ peaks would be expected. The absence of peaks there suggests that $\mathrm{HCl}$ was successfully eliminated from the reaction flask, and that $\mathrm{Cl}$ was not incorporated in the polymeric structure. The two rightmost arrows in the figure correspond to the energies where characteristic Al peaks are expected. As can be seen, there are relatively small peaks located in these regions, which are attributed to the Al substrate on which the samples were prepared. The $\mathrm{C} 1 \mathrm{~s}$ peak located at $289 \mathrm{eV}$ is commonly attributed to adventitious carbon due to short-term exposure of the sample to ambient air during sample preparation. ${ }^{98}$

The remaining peaks in the XP spectrum can be attributed to signals resulting from the polymer itself. The two peaks at $\sim 980$ and $\sim 533 \mathrm{eV}$ correspond to the $\mathrm{O} \mathrm{KL}_{1} \mathrm{~L}_{2,3}$ 


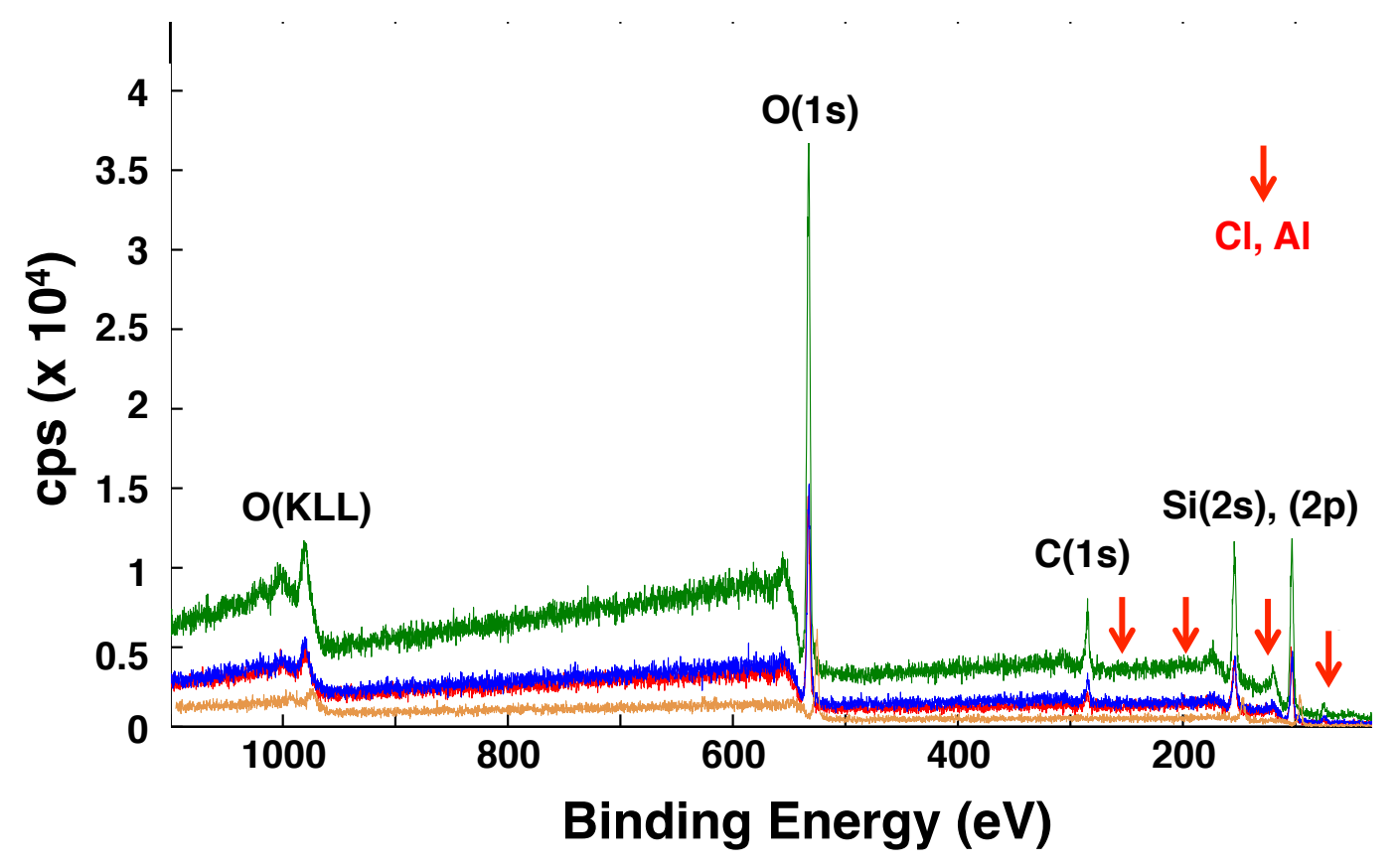

Figure 2.2. XPS survey scan spectra of the dry polymer, measured in four different regions of the same sample. Characteristic peaks are labeled by atomic orbital. Locations indicated by red arrows correspond to absent $\mathrm{Cl}$ (left two) and $\mathrm{Al}$ substrate (right two) binding energies.

and $\mathrm{O} 1 \mathrm{~s}$ regions, respectively. The former peak results from the emission of an electron from the $\mathrm{L}_{2,3}$ orbital, which occurs as a result of the emission of energy from the $\mathrm{K}$ to $\mathrm{L}_{1}$ transition. In addition, the two peaks at $\sim 154$ and $\sim 103 \mathrm{eV}$ correspond to the $\mathrm{Si} 2 \mathrm{~s}$ and $\mathrm{Si}$ $2 p$ binding energies, respectively. A more detailed analysis of the Si $2 p$ region is shown in Figure 2.3. The peak was best fit by two components. The first is the most intense, located at $103.2 \mathrm{eV}$, and can be attributed to a suboxide of silicon (e.g., $\mathrm{Si}^{\mathrm{n+}}$, where $\mathrm{n}<4$ ). The second peak, located at $104.8 \mathrm{eV}$, can be attributed to heavily oxidized silicon (e.g., $\left.\mathrm{Si}^{4+}\right)$. The latter peak is almost 10 -fold less intense and appears as a small shoulder on the high energy side of the major peak in the measured spectrum. These peak positions are in good agreement with the predicted oxidation states of silicon in a suboxide polymer. 


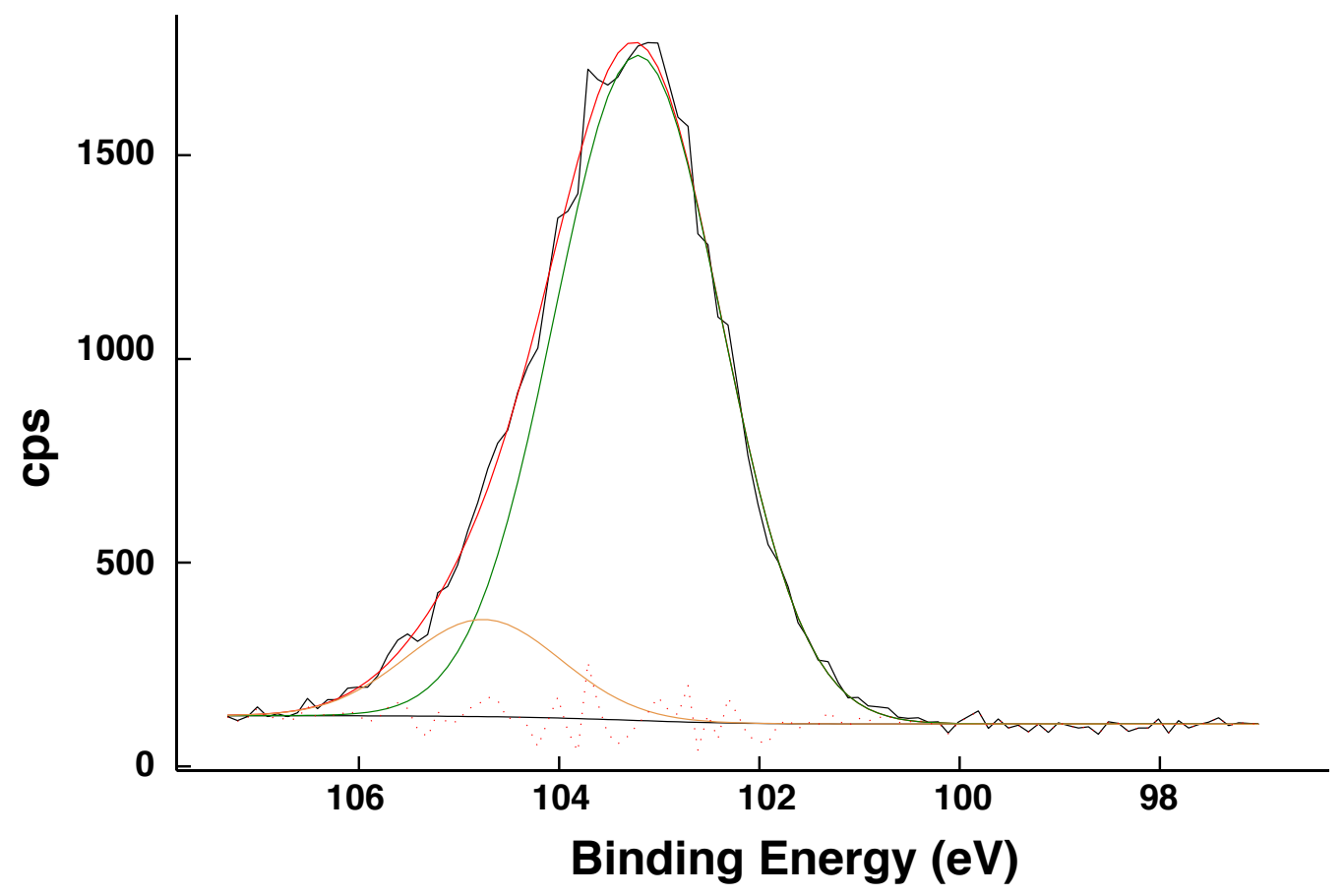

Figure 2.3. Si $2 \mathrm{p}$ region of the XP spectrum of the dry polymer sample prior to annealing. The measured data (black) was fit by two peaks at 103.2 (green) and 104.8 (orange) $\mathrm{eV}$. The calculated peak (red) results from the addition of the two fitted peaks. The pale red dots correspond to residuals from the fit.

The measured percent compositions of oxygen and silicon in the dry polymer were calculated from the survey spectra (Fig. 2.2) to be 51.7 and 29.5 atomic\%, respectively. Based on the empirical formula of the polymer, $\left(\mathrm{HSiO}_{1.5}\right)_{\mathrm{n}}$, it was expected that the concentration of oxygen would be 1.5 times greater than the concentration of $\mathrm{Si}$ in the sample. However, the concentration of oxygen was determined from the data in Figure 2.2 to be 1.75 times greater than silicon. Discrepancies in the expected and experimental results may be due to the presence of an oxide layer on the surface of the aluminum disk, as well as $\mathrm{C}-\mathrm{O}$ bonding in the adventitious carbon species. Both of these sources of error would result in an expected oxygen to silicon ratio that is greater than $1.5: 1$. 


\subsubsection{Thermal processing of $\left(\mathrm{HSiO}_{1.5}\right)_{n}$}

The formation of Si NPs from the high temperature processing of silicon suboxides under an inert atmosphere has been studied extensively. ${ }^{9,21,91,99-107}$ The suboxide precursor that is used most commonly in the literature for the production of Si NPs is prepared from hydrolyzed, polycondensed hydrogen silsesqioxane (HSQ). The resulting $\mathrm{HSiO}_{1.5}$ polymer has been suggested to react at high temperatures according to the mechanism shown in Scheme 2.2. ${ }^{21}$ In the first stage of the reaction, at temperatures above $250^{\circ} \mathrm{C}$, four equivalents of $\mathrm{HSiO}_{1.5}$ disproportionate into $\mathrm{SiH}_{4}$ and three molar equivalents of $\mathrm{SiO}_{2}$. At temperatures above $350^{\circ} \mathrm{C}$, the $\mathrm{SiH}_{4}$ produced in the first stage will decompose into amorphous, elemental silicon clusters (a-Si) and hydrogen gas is evolved. However, if the second stage occurs below $350^{\circ} \mathrm{C}$, decomposition of $\mathrm{SiH}_{4}$ will not occur. As a result, $\mathrm{SiH}_{4}$ will evolve from the reaction as a gas, which reduces the yield of elemental Si from the synthesis. Finally, the third stage occurs when reaction temperatures exceed $900^{\circ} \mathrm{C}$, where a-Si will undergo phase conversion into crystalline silicon (c-Si or Si NPs).

$$
\begin{aligned}
& \text { 1. } 4 \mathrm{HSiO}_{1.5} \longrightarrow 3 \mathrm{SiO}_{2}+\mathrm{SiH}_{4} \\
& \text { 2. } \mathrm{SiH}_{4} \longrightarrow a-\mathrm{Si}+2 \mathrm{H}_{2} \\
& \text { 3. } a-\mathrm{Si} \longrightarrow c-\mathrm{Si}
\end{aligned}
$$

Scheme 2.2. Balanced chemical equations showing formation of $\mathrm{Si}^{0}$ from $\mathrm{HSiO}_{1.5}$ at high temperatures.

The maximum holding temperature has been shown by XRD and TEM to have an effect on the average size of the Si NPs produced from the annealing of HSQ-derived $\left(\mathrm{HSiO}_{1.5}\right)_{\mathrm{n}}$, where higher temperatures produce larger Si NPs $(3-18 \mathrm{~nm} \mathrm{Si} \mathrm{NPs} \mathrm{from}$ 


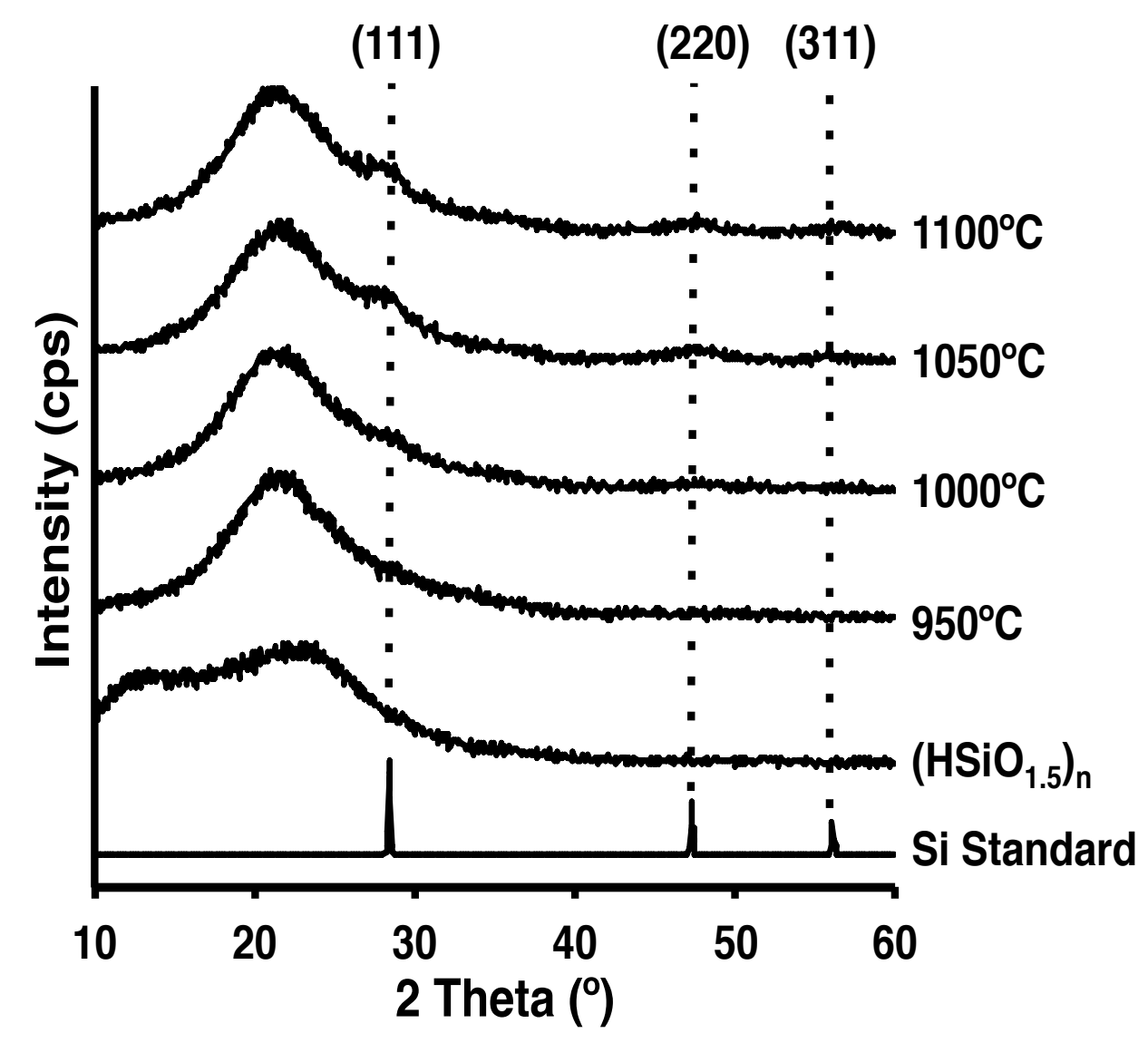

Figure 2.4. XRD patterns of $\left(\mathrm{HSiO}_{1.5}\right)_{\mathrm{n}}$ annealed for $10 \mathrm{~h}$ at the indicated temperatures between 950 and $1100^{\circ} \mathrm{C}$. Reference patterns of $\left(\mathrm{HSiO}_{1.5}\right)_{\mathrm{n}}$ and a $\mathrm{Si}$ standard are shown. Peak positions expected for c-Si are indicated by the dotted lines.

annealing at $\left.1100^{\circ}-1350{ }^{\circ} \mathrm{C}\right) .{ }^{9,21,99}$ For the $\mathrm{HSiCl}_{3}$-derived $\left(\mathrm{HSiO}_{1.5}\right)_{\mathrm{n}}$, we studied the effect of a change in the maximum processing temperature on the average size of Si NPs, as well as the estimated mass yield of Si NPs, by XRD. Figure 2.4 contains the XRD patterns of $\mathrm{HSiCl}_{3}$-derived $\left(\mathrm{HSiO}_{1.5}\right)_{\mathrm{n}}$ after annealing for $10 \mathrm{~h}$ at the indicated temperatures between 950 and $1100^{\circ} \mathrm{C}$. XRD patterns of the dry polymer prior to annealing and a silicon standard are shown in Figure 2.4 for reference.

Prior to any heat treatment, the XRD pattern of the dry polymer contains two broad features that are typical of amorphous powders. When the polymer was annealed at 
Table 2.1. Effect of maximum annealing temperature on average Si NP size and crystallinity for samples annealed for $10 \mathrm{~h}$.

\begin{tabular}{ccc}
\hline Max. Temp. $\left({ }^{\circ} \mathrm{C}\right)$ & Si NP size $(\mathrm{nm})$ & Crystallinity (\%) \\
\hline 950 & - & - \\
1000 & 4 & 5 \\
1050 & 3 & 10 \\
1100 & 3 & 10 \\
\hline
\end{tabular}

$950^{\circ} \mathrm{C}$, the shape and position of the amorphous peak was slightly changed $v$. the unheated powder; however, peaks belonging to crystalline silicon were indiscernible. When the maximum annealing temperature was increased to $1000^{\circ} \mathrm{C}$, broad peaks with low intensity at $28^{\circ}, 46^{\circ}$, and $55^{\circ} 2 \theta$ became discernible, and are consistent with c-Si. These peaks became more distinct as the annealing temperature was increased further to $1100^{\circ} \mathrm{C}$. It is to be expected that even higher temperatures would elicit greater conversion to crystalline silicon from the annealed polymer; however, the tube furnace used in this study is limited to $1100^{\circ} \mathrm{C}$.

For samples in which the crystalline silicon (c-Si) phase was discernible from the large amorphous features, the average Si NP diameter (d) and percent crystallinity of each sample were estimated. First, the average size of nanocrystalline domains can be estimated by XRD by analyzing the diffraction peak broadening with the Scherrer equation,

$$
\tau=\frac{\kappa \lambda}{\beta \cos (\theta)}
$$

where $\tau$ is the nanocrystal diameter, $\kappa$ is the shape factor ( 0.89 for spherical NPs), $\lambda$ is the wavelength of the diffracted beam $(\mathrm{Cu} \mathrm{K \alpha}=0.154 \mathrm{~nm}), \beta$ is the full-width at half max 
(FWHM) of the diffraction peak (in radians), and $\theta$ is the peak position of the diffracted beam (20/2, in radians). According to the results shown in Table 2.1, the estimated Si NP diameter does not show a strong correlation to the maximum processing temperature. However, due to the difficulty in determining the c-Si peak widths from the amorphous background in the data shown in Figure 2.4, the estimated Si NP size cannot be regarded as reliable.

Next, the percent crystallinity of a sample was estimated by calculating the ratio of the integrated peak areas of the c-Si phase to the overall integrated area of the calculated, or best fit, pattern. Table 2.1 illustrates the effect of maximum processing temperature on the estimated crystallinity of the annealed powders shown in Figure 2.4. When annealed at $1100^{\circ} \mathrm{C} v s .1000^{\circ} \mathrm{C}$, the estimated percent crystallinity of the product increased from $5 \%$ to $10 \%$, which is consistent with expectations for raising the reaction temperature. Determination of the percent crystallinity of the sample that was annealed at $950^{\circ} \mathrm{C}$ for $10 \mathrm{~h}$ by the same method as the other samples was not possible due to the lack of obvious features attributable to crystalline silicon domains in the XRD pattern.

Table 2.2. Effect of annealing time on average Si NP size and crystallinity for samples annealed at $1100{ }^{\circ} \mathrm{C}$.

\begin{tabular}{ccc} 
Time at $1100^{\circ} \mathbf{C}$ & Si NP size (nm) & Crystallinity (\%) \\
\hline 5 min & 2 & 20 \\
$30 \mathrm{~min}$ & 3 & 20 \\
$90 \mathrm{~min}$ & 2 & 20 \\
$\mathbf{1 0 ~ h}$ & 3 & 10 \\
\hline
\end{tabular}


Furthermore, the same uncertainty that is present in the calculated Si NP diameters is present for the calculated percent cystallinities, as a best fit pattern was used to calculate both.

For a given temperature, the time that the samples were held at that temperature was varied and XRD used to monitor the degree of crystallinity and Si NP diameter as a function of reaction time. As can be seen in Table 2.2, a maximum conversion of the polymer into c-Si is estimated to be reached at $1100^{\circ} \mathrm{C}$ within the first hour of annealing, and subsequently decreases with longer annealing times. Similar results were observed for samples annealed at $1050^{\circ} \mathrm{C}$, (data not included). Overall, the observed qualitative trends from the XRD data would suggest that standard annealing times of less than 120 min should be used in the future and that annealing should be conducted at the maximum possible furnace temperature. Finally, for comparison against the percent crystallinites in this section, which can be regarded as percent conversion to $\mathrm{Si}^{0}$ domains, the theoretical conversion of $\mathrm{Si}^{0}$ from the polymer is $25 \%$ according to the mechanism shown in Scheme 2.2.

Given the data shown in Figure 2.4, it is not possible to make definitive conclusions regarding the effect of annealing conditions on average crystallite size or percent crystallinity. The results estimated in Tables 2.1 and 2.2 have a high margin of error associated with them due to the subjectivity of manual peak fitting of the crystalline phase peak width, since the $100 \%$ peak of the elemental silicon phase $((111)$ at $28^{\circ} 2$-theta) is convoluted with the prevalent scattering peak of the amorphous oxide. However, although Scherrer analysis of the XRD data is unreliable, the bright red PL observed from the powder after annealing under illumination with a $365 \mathrm{~nm}$ handheld 
UV-lamp would be consistent with $\sim 3 \mathrm{~nm}$ particles as predicted by the effective mass approximation (EMA).

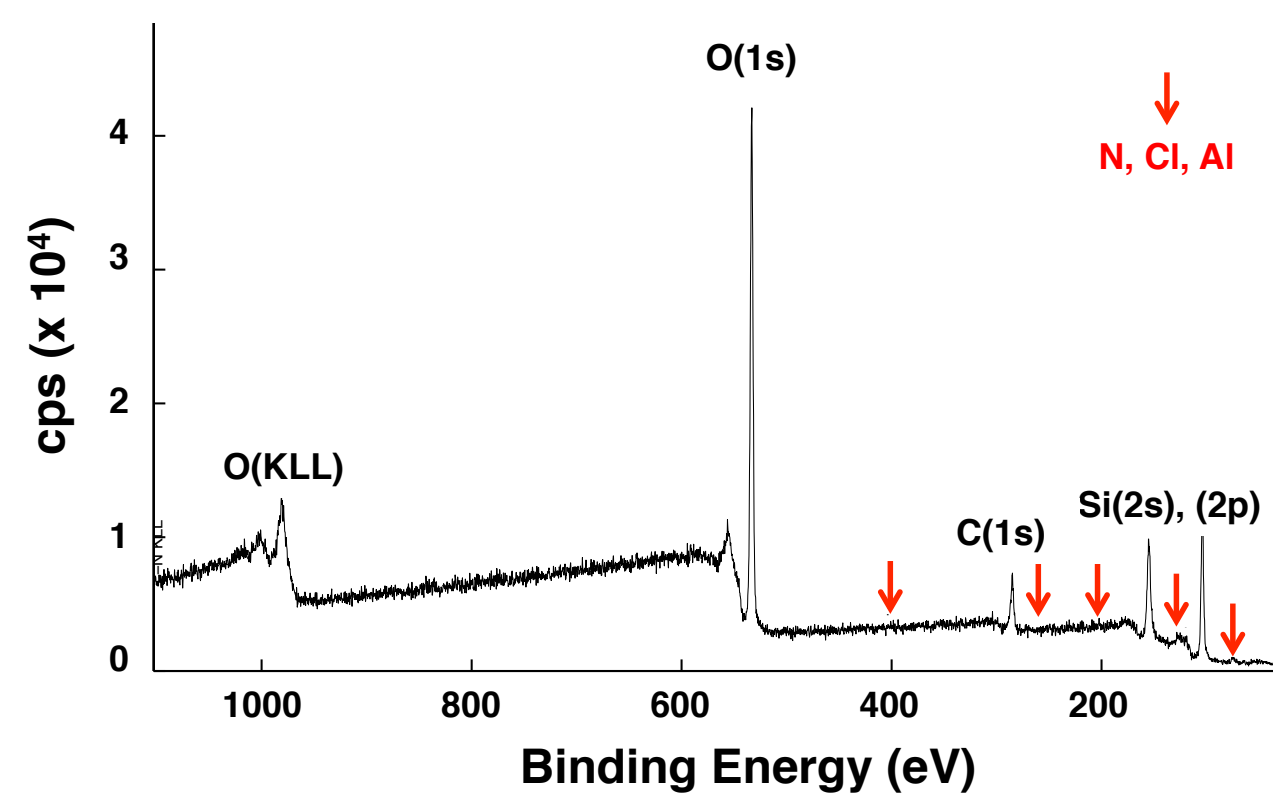

Figure 2.5. Representative XPS survey spectrum of the polymer following annealing at $1100{ }^{\circ} \mathrm{C}$ for $10 \mathrm{~h}$ under flowing $\mathrm{N}_{2}$. Characteristic peaks are labeled by atomic orbital. Locations of $\mathrm{N}$ (leftmost arrow), $\mathrm{Cl}$ (middle two arrows) and $\mathrm{Al}$ (rightmost two arrows) binding regions are indicated by red arrows.

For the studies presented in Chapters 3 and 4 , the $\mathrm{HSiCl}_{3}$-derived polymer was annealed at $1100^{\circ} \mathrm{C}$ for $10 \mathrm{~h}$. This was the standard protocol that was established prior to this work, and these parameters were maintained such that results could be comparable. The changes in the composition of the polymer as a result of these annealing conditions were characterized by XPS. A survey spectrum, collected from $1100-0 \mathrm{eV}$, is shown in Figure 2.5. The leftmost arrow in the figure indicates the energy range where a characteristic N 1s peak would be expected. There are reports in the literature that suggest that annealing of silicon suboxides in an $\mathrm{N}_{2}$ atmosphere may result in the formation of $\mathrm{Si}-\mathrm{N}$ type defects or $\mathrm{Si}-\mathrm{N}$ bonding at the $\mathrm{Si}_{-} \mathrm{SiO}_{2}$ interface. ${ }^{57,108}$ However, 


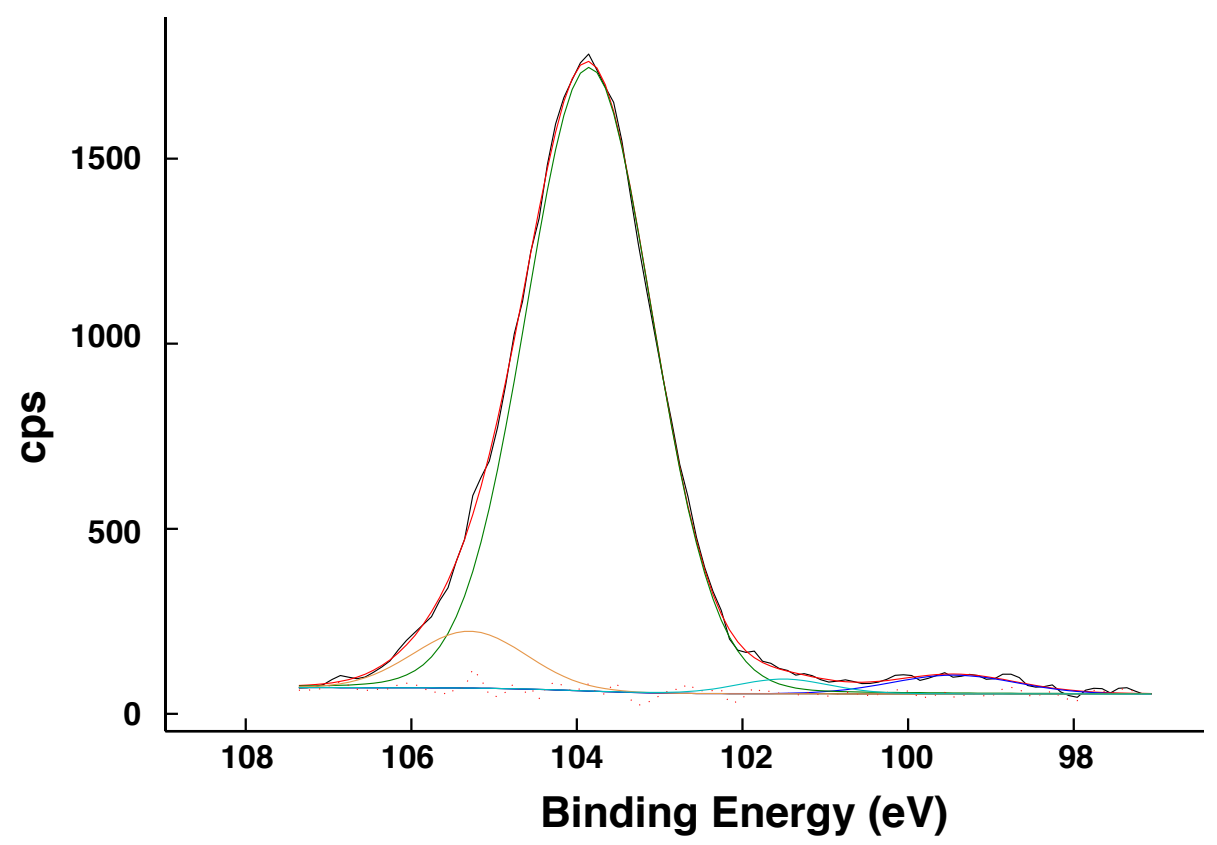

Figure 2.6. Si $2 \mathrm{p}$ region of the XP spectrum of polymer annealed at $1100{ }^{\circ} \mathrm{C}$ for $10 \mathrm{~h}$ under flowing $\mathrm{N}_{2}$. The measured data (black) was fit by four peaks at 99.5 (blue), 101.5 (teal),103.85 (green) and 105.3 (orange) eV. The calculated peak (red) results from the addition of the four fitted peaks. The pale red dots correspond to residuals from the fit.

the absence of a $\mathrm{N}$ 1s peak in Figure 2.5 (leftmost red arrow) suggests that either $\mathrm{N}$ was not incorporated into the structure of the solid or is present below the limit of detection. The second and third arrows from the left in the figure correspond to the regions where characteristic $\mathrm{Cl}$ peaks are expected, which are again absent from the spectrum. The rightmost two arrows in the figure indicate two peaks that are attributed to the $\mathrm{Al}$ substrate on which the samples were prepared. Finally, the C 1s peak owing to adventitious carbon that was present in Figure 2.2 is also present in Figure 2.5. Again, the peaks corresponding to oxygen and silicon binding energies are attributed to the annealed polymer itself. The peaks at $\sim 980$ and $\sim 533 \mathrm{eV}$ correspond to the $\mathrm{O} \mathrm{KL}_{1} \mathrm{~L}_{2,3}$ and $\mathrm{O} 1 \mathrm{~s}$ binding energies, respectively. The two peaks at $\sim 154$ and $\sim 103 \mathrm{eV}$ correspond to the $\mathrm{Si}$ $2 \mathrm{~s}$ and $\mathrm{Si} 2 \mathrm{p}$ binding energies, respectively. 
A more detailed spectrum of the Si $2 p$ peak is shown in Figure 2.6. The best fit of the measured data contains four peak components, in contrast to the two components fit to the Si $2 p$ spectrum from the polymer prior to annealing (Fig. 2.3). The most intense peak is located at $103.85 \mathrm{eV}$ and is indicative of highly oxidized silicon (nearly 4+). Two peaks with low intensity at 105.3 and $101.5 \mathrm{eV}$ are likewise indicative of more highly and less highly oxidized silicon atoms, respectively. Finally, there is a small peak at $99.5 \mathrm{eV}$ that was not present in the dry polymer before annealing. This peak supports the formation of $\mathrm{Si}^{0}$ domains within the oxidized matrix. Only $3 \%$ of the integrated peak area of the $\mathrm{Si} 2 \mathrm{p}$ region is attributed to elemental silicon, which is low relative to the $\sim 10 \%$ crystallinity roughly estimated by XRD (see Tables 2.1 and 2.2 ). This could be due to the large uncertainty in the XRD analysis, or to differences in the depth of penetration of the incident X-rays between the two techniques (XPS unable to penetrate a thick a-SiO 2 layer).

The measured percent compositions of oxygen and silicon in the dry polymer were calculated from the survey spectra (Fig. 2.5) to be 56.5 and $15.0 \%$, respectively. The reduced ratio of silicon to oxygen suggests that $\mathrm{SiH}_{4}$ may have been lost throughout the annealing process before it could undergo decomposition within the solid matrix.

\subsubsection{Liberation of Si NPs from oxide matrix}

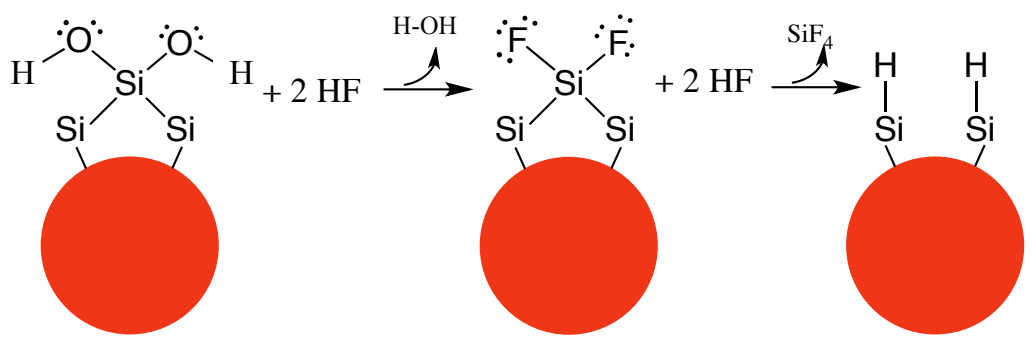

Scheme 2.3. Mechanism of HF etching of an oxidized silicon surface. 
To isolate the Si NPs from the oxide matrix, the annealed polymer is etched in a mixture of $5.0 \mathrm{~mL}$ each of $\mathrm{HF}$, abs-EtOH and $\mathrm{H}_{2} \mathrm{O}$. The HF preferentially reacts with the surface Si-O bonds, resulting in surface Si-F bonds and loss of $\mathrm{H}_{2} \mathrm{O}$. The resulting Si-F surface bonding causes greater polarization of the underlying Si-Si bonds, making it possible for another two equivalents of HF to react. After reaction with additional equivalents of $\mathrm{HF}, \mathrm{SiF}_{4}$ is lost, taking away atoms from the first atomic layer and leaving the second atomic Si layer H-passivated. ${ }^{17,109}$ This process is summarized below in Scheme 2.3.

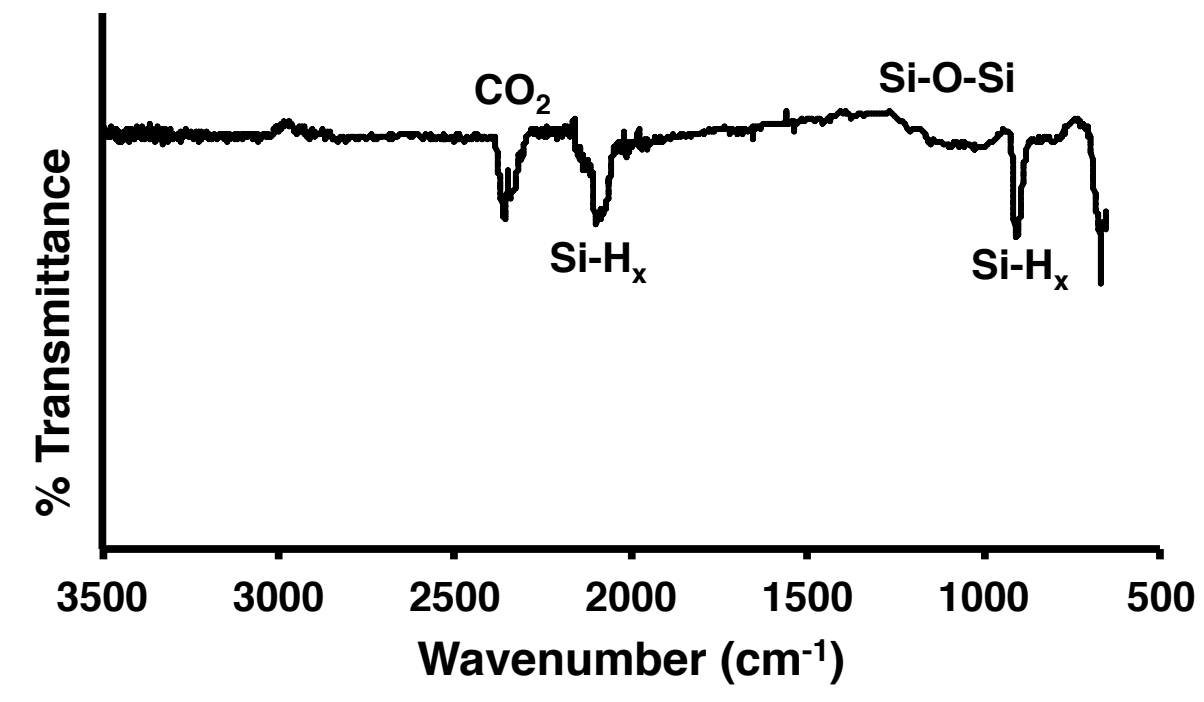

Figure 2.7. FT-IR spectrum of $\mathrm{H}-\mathrm{Si}$ NPs immediately following extraction from a 60 min aqueous $\mathrm{HF}$ etch.

After the oxide layer is etched away, the surface of the Si NPs are H-passivated (H-Si NPs), which renders the particles soluble in non-polar solvents such as hexane or toluene. A simple biphasic extraction can then be conducted in order to transfer the H-Si NPs from the aqueous etchant into hexane, toluene, etc.. Figure 2.7 contains a representative FT-IR spectrum of H-Si NPs extracted into hexane immediately following 
extraction from a 60 min etch. The peaks at 2070 and $890 \mathrm{~cm}^{-1}$ both correspond to Si-H bonding, which confirms successful H-passivation of the surface by the HF. Additionally, there is some absorbance intensity in the region that is typically assigned to Si-O-Si bonding, which suggests that the H-Si NP surface is partially oxidized. Finally, there is a peak at $2325 \mathrm{~cm}^{-1}$; this is occasionally present in the FT-IR spectra of H-Si NPs and can be attributed to atmospheric $\mathrm{CO}_{2}$.

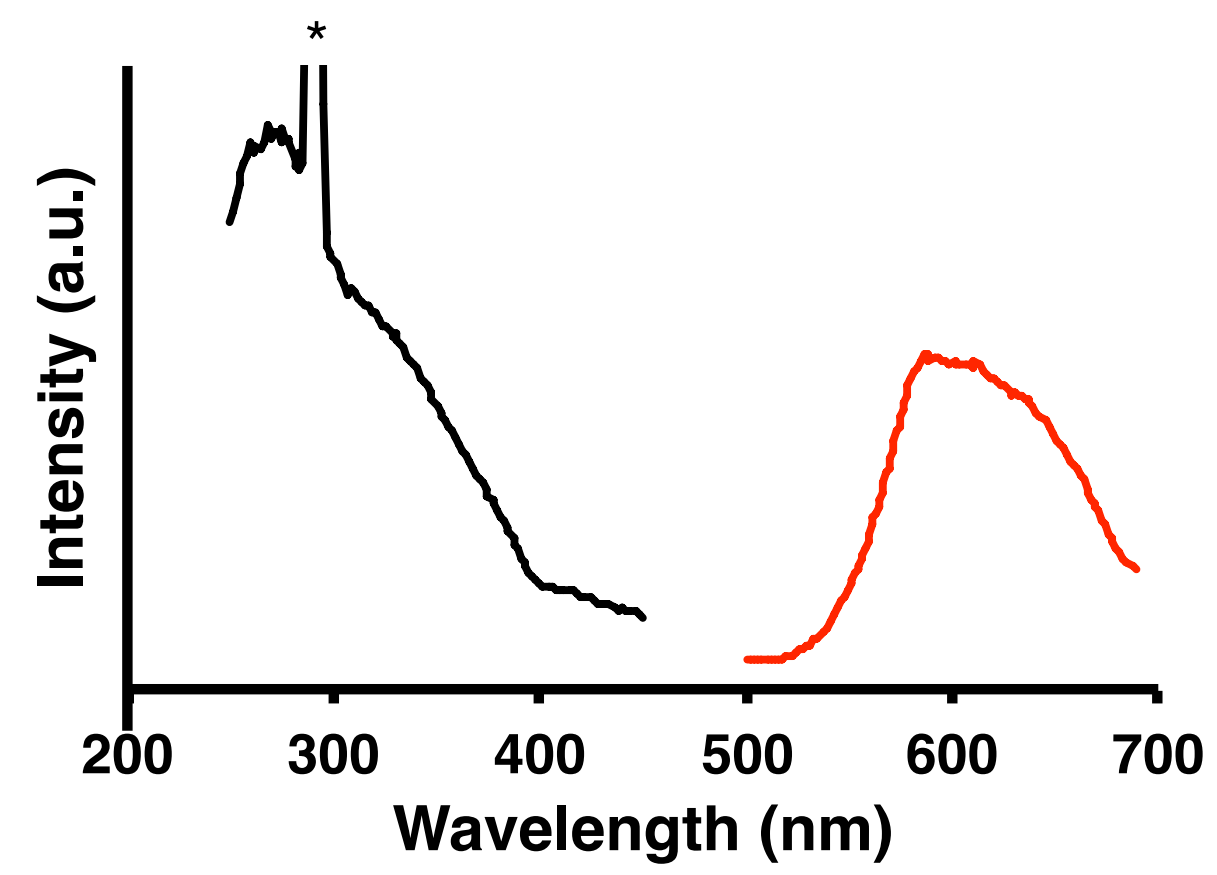

Figure 2.8. PL (red line, $\lambda_{\mathrm{exc}}=360 \mathrm{~nm}$ ) and PLE (black line, $\lambda_{\mathrm{em}}=590 \mathrm{~nm}$ ) spectra of H-Si NPs in hexane. Scattering peak marked with *.

When polymer annealed for $10 \mathrm{~h}$ at $1100{ }^{\circ} \mathrm{C}$ is etched for $60 \mathrm{~min}$, H-Si NPs with intense, visible red PL (590 - $700 \mathrm{~nm})$ are consistently produced. Figure 2.8 contains the PL and PLE spectra of a representative suspension of H-Si NPs in hexane. The break in the PLE spectrum (black trace) is due to the elimination of a scattering peak. When excited at $360 \mathrm{~nm}$, the resulting PL spectrum contains a peak ranging from 

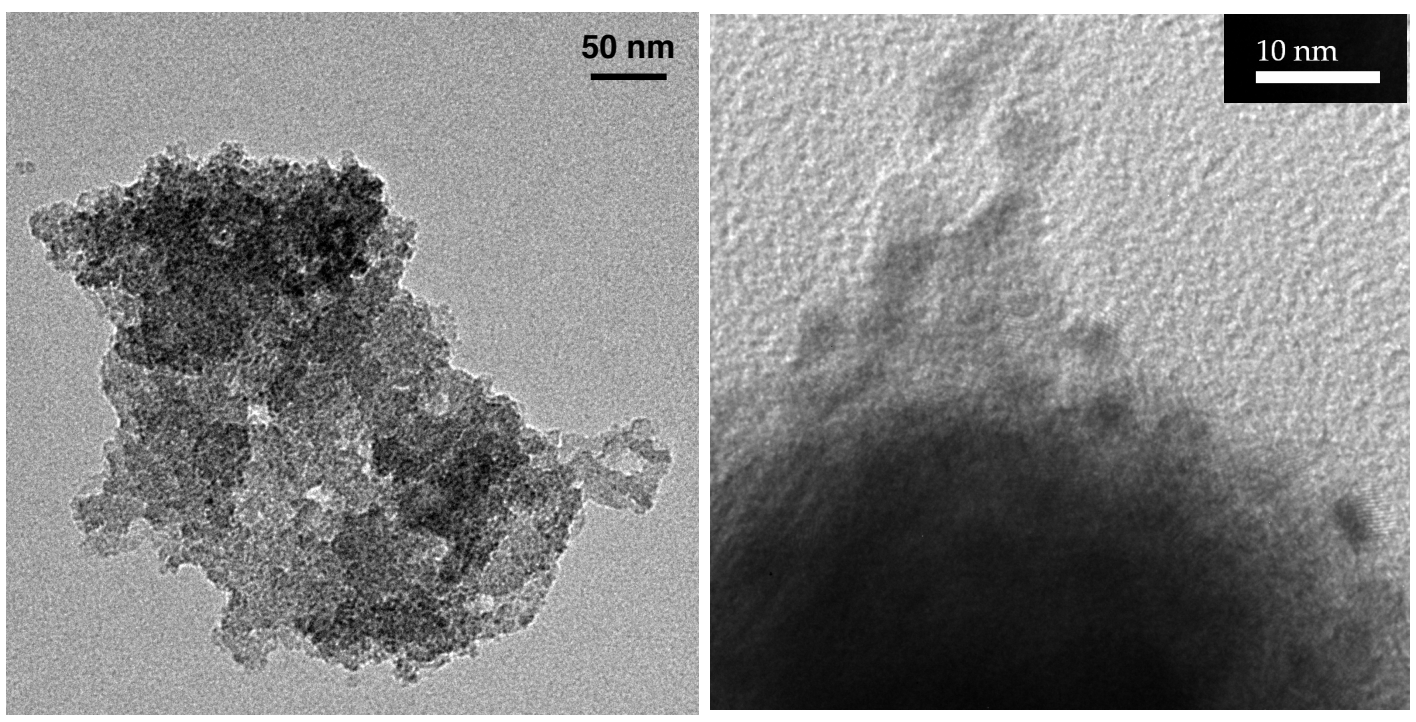

Figure 2.9. Representative TEM images of H-Si NP aggregate structures. Scale bars (left) $50 \mathrm{~nm}$ and (right) $10 \mathrm{~nm}$.

$\sim 550-750 \mathrm{~nm}$, with an emission maximum at $\sim 590 \mathrm{~nm}$. When monitoring for $590 \mathrm{~nm}$ emission, the corresponding PLE spectrum contains a broad peak that ranges from $250-400 \mathrm{~nm}$, with maximum excitation intensity at $260 \mathrm{~nm}$.

It is important to note that the Si NPs are prone to aggregation and precipitation. This is especially true for H-Si NPs in low boiling point solvents having low viscosity, such as hexane. Figure 2.9 shows a TEM image of H-Si NPs prepared by: (left) dropcasting a hexane suspension onto a $\mathrm{Cu}$ grid and (B) dipping a $\mathrm{Cu}$ grid into a hexane suspension. As can be seen, the crystalline domains are contained within larger aggregate structures rather than isolated in space (Fig. 2.9 right figure). We determined that the use of toluene as a storage solvent or co-solvent aids with short-term suspension stability, increasing the stability of a diluted suspension of H-Si NPs in hexane from a few minutes to a few hours. The surface composition of the Si NPs is likewise unstable and prone to reactivity. For example, we have observed that the H-Si NPs have a tendency to cross- 
link with glassware. The greater implications for the instability of H-Si NPs is the subject of this dissertation and will be discussed in the chapters that follow. 


\section{Chapter 3}

Hydrosilylation of silicon nanoparticles in $n$-alkane solvents:

implication of radicals in the chemical reactivity of hydride-passivated surfaces

\subsection{Introduction}

The stability of the hydride-passivated silicon nanoparticle (H-Si NP) surface at mildly elevated temperatures was analyzed. Upon heating in refluxing $n$-hexane (b.p. $=68^{\circ} \mathrm{C}$ ), evidence for successful hydrosilylation (HS) of the Si NP surface was observed by infrared spectroscopy, in combination with varying degrees of surface oxidation. The unexpected reactivity of the Si surface in $n$-hexane supports the unstable nature of the H-Si NP surface, and furthermore implicates the presence of highly-reactive Si radicals on the surfaces of the Si NPs. We propose that reaction of alkene impurities with the Si surface radicals is largely responsible for the observed HS. However, we also present a mechanism by which Si surface radicals could react with alkanes, ultimately resulting in surface alkylation.

\subsection{Motivation for the present study}

It is generally accepted that hydrosilylation (HS) in the absence of radical initiators can be initiated by the homolytic cleavage of the $\mathrm{Si}-\mathrm{H}$ bond at temperatures greater than $150^{\circ} \mathrm{C} .^{81,83-86}$ Alternatively, UV light of appropriate energy $(\sim 3.5 \mathrm{eV})$ can also be used to initiate homolytic cleavage of the Si-H bond. ${ }^{87}$ Such conditions are often

used to accomplish thermal HS reactions with a variety of alkenes and alkynes. ${ }^{81-88}$ Scheme 3.1 depicts the mechanism by which passivation of the $\mathrm{Si}-\mathrm{H}$ surface with terminal alkenes is thought to occur. In (1), either UV light or heat is used to cause 
homolytic cleavage of the Si-H bond, generating a Si-based surface radical. The silicon radical can then react with the unsaturated bond of an alkene or alkyne, which are typically in high excess in comparison to the $\mathrm{Si}-\mathrm{H}$ bonds, as shown in (2); this generates a reactive carbon radical. As shown in (3), the carbon radical can abstract a hydride from a neighboring Si-H bond, thereby re-generating a Si-based surface radical. The silicon radical can then go on to propagate the surface passivation by reacting with another alkene molecule, as shown in (4), thereby re-generating a carbon based radical species, as shown in (5). Propagation of the passivation of the surface then continues via repetition of steps (3) and (4).

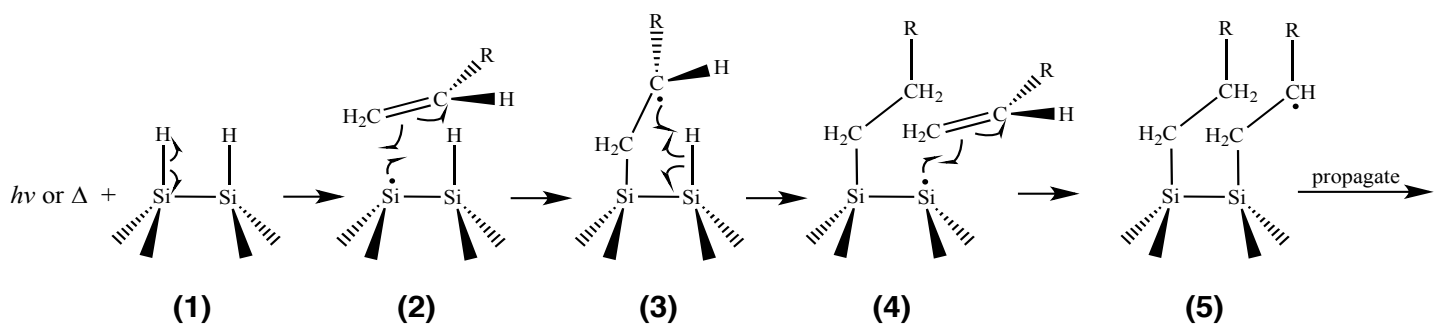

Scheme 3.2. Commonly accepted mechanism for the hydrosilylation of the $\mathrm{Si}-\mathrm{H}$ surface with terminal alkenes.

Passivation of the H-Si NP surface with large alkenes or alkynes is limited by steric hindrance of the ligand at the particle surface. This means that it is not possible to passivate $100 \%$ of the surface Si sites. In the case of 1-decene, one study suggested that the surface coverage was as low as $30-50 \%$ Si-C bonding. ${ }^{83}$ Such low surface coverage would be expected to result in the persistence of reactive sites (e.g., Si surface radicals or carbon radicals) after alkylation. For example, in high concentrations of alkene or alkyne, it has been proposed that the formation of reactive carbon-based radicals on the alkylated Si NPs may promote rapid oligomerization of un-bound the alkene or alkyne in 
solution. ${ }^{110}$ However, in the absence of excess alkene or alkyne, such as following isolation and suspension of the alkyl-passivated Si NPs in storage solvents, it is possible that radicals may also promote the desorption of the surface ligand, as we observed in a prior study. ${ }^{67}$

The mechanism shown in Scheme 3.1 makes several important assumptions: 1) that either high temperature $\left(\mathrm{T} \geq 150^{\circ} \mathrm{C}\right)$ or UV-light $(\mathrm{h} v \leq 350 \mathrm{~nm})$ is necessary to generate reactive Si surface sites to initiate HS, 2) that all of the Si surface sites are initially hydride passivated, and 3) that an unsaturated ligand is required to passivate the surface. However, due to the relatively low thermodynamic stability of the Si-H bond (vs. Si-O $)^{55}$ and the hydride-abstraction capability of trace $\mathrm{O}_{2},{ }^{111}$ we hypothesized that there could be a large number of reactive surface sites present on H-Si NPs at room temperature and under ambient light. These highly-reactive surface sites would be expected to react with alkenes and alkynes, resulting in some degree of HS of the Si NP surface under mild surface conditions. However, we argue that some degree of HS may also be possible for H-Si NPs suspended in pure alkane solvents, due to the hydrideabstraction capability of silicon surface radicals. Herein, we tested the reactivity of minimally oxidized H-Si NPs towards $95+\% n$-hexane, a common storage solvent for H-Si NPs, to probe the extent of the stability of the H-Si NP surface under mild heating.

\subsection{Experimental}

\subsubsection{Materials}

Trichlorosilane $\left(\mathrm{HSiCl}_{3}, 98 \%\right.$, Alfa Aesar, Ward Hill, MA), absolute ethanol (abs-EtOH, anhydrous, 200-proof, Pharmco-AAPER, Brookfield, CT), ethanol (EtOH, 
ACS grade, stockroom), hydrofluoric acid (HF, ACS reagent, 48-51\% solution in water, Acros Organics, NJ), sulfuric acid $\left(\mathrm{H}_{2} \mathrm{SO}_{4}, 95\right.$ - $98 \%$ ACS reagent, Sigma-Aldrich, St. Louis, $\mathrm{MO})$, and sodium bicarbonate $\left(\mathrm{NaHCO}_{3}\right.$, stockroom) were purchased and used as received. Millipore water (18 $\mathrm{M} \Omega \bullet \mathrm{cm}$ resistivity) was used during the polycondensation and etching procedures to prepare the Si NPs. Laboratory deionized water $\left(\mathrm{DI} \mathrm{H}_{2} \mathrm{O}\right)$ was used during the purification of $n$-hexane.

$n$-Hexane $\left(\mathrm{C}_{6} \mathrm{H}_{14}, 95+\%\right.$, Acros Organics, Fair Lawn, NJ) was purchased and was used either as-received or following purification, as specified. In order to eliminate alkene impurities from the as-received $n$-hexane, purification was accomplished as follows: $300 \mathrm{~mL}$ of hexane was shaken in a separatory funnel with $5 \mathrm{~mL}$ aliquots of concentrated sulfuric acid (2-7 aliquots), followed by $10 \mathrm{~mL}$ of deionized water. The $n$-hexane was then shaken with $10 \mathrm{~mL}$ of $10 w t \% \mathrm{NaHCO}_{3}$ to neutralize residual sulfuric acid, followed by two additional washes with $10 \mathrm{~mL}$ of $\mathrm{DI} \mathrm{H}_{2} \mathrm{O}$. Both the as-received and purified hexane were stored over $4 \AA$ molecular sieves prior to use.

\subsubsection{Preparation of $\mathrm{H}-\mathrm{Si} \mathrm{NPS}$}

H-Si NPs were produced according to a previously published protocol, ${ }^{23}$ which has been discussed in detail in Chapter 2. Briefly, $\left(\mathrm{HSiO}_{1.5}\right)_{\mathrm{n}}$ was prepared through polycondensation and polymerization of $\mathrm{HSiCl}_{3}$ with $\mathrm{H}_{2} \mathrm{O}$, and dried at $100^{\circ} \mathrm{C}$ under flowing Ar. Approximately $0.5 \mathrm{~g}$ of the resulting white polymer was loaded into a $5 \mathrm{~mL}$ alumina combustion boat (Coors brand) and then annealed at $1100^{\circ} \mathrm{C}$ for $10 \mathrm{~h}$ under flowing $\mathrm{N}_{2}$. The resulting product was a light brown powder that exhibited red PL under irradiation by a $365 \mathrm{~nm}$ handheld lamp directly following annealing. Next, $0.45-0.55 \mathrm{~g}$ of the product was added to a PTFE sample cup equipped with a magnetic stir bar, to 
which $5 \mathrm{~mL}$ of abs-EtOH and $5 \mathrm{~mL}$ of $\mathrm{H}_{2} \mathrm{O}$ was added, and the solid was dispersed by sonication. Then, $5 \mathrm{~mL}$ of HF was added and the mixture was left to stir loosely covered for $60 \mathrm{~min}$. The nanoscale $\mathrm{Si}^{0}$ domains were liberated from the oxidized matrix as H-passivated Si NPs, which were phase-transferred into $40 \mathrm{~mL}$ of hexane and stored under Ar until further use. Samples were studied by FT-IR and PL spectroscopies prior to subsequent surface-functionalization attempts.

\subsubsection{Purification of partially-oxidized H-Si NPS}

In some cases, we observed that the H-Si NPs exhibited surface oxidation by FT-IR spectroscopy immediately following extraction from the etch. When this occurred, a purification step was conducted in order to eliminate partially oxidized domains from the H-Si NP suspension prior to subsequent surface modification attempts. Post-etch purification of oxidized H-Si NPs was accomplished as follows: first, $10 \mathrm{~mL}$ of the $40 \mathrm{~mL}$ stock in purified hexane was split into two $5 \mathrm{~mL}$ aliquots, transferred to $15 \mathrm{~mL}$ glass centrifuge tubes, and $5 \mathrm{~mL}$ of EtOH was added to each. The suspensions were then centrifuged at 2,000 rpm for $10 \mathrm{~min}$, and the supernatant discarded. Next, $5 \mathrm{~mL}$ of EtOH was added to each tube, which were then sonicated and centrifuged at 2,000 rpm for $10 \mathrm{~min}$ and the supernatant discarded. Next, $5 \mathrm{~mL}$ of purified hexane was added to each tube, and the samples were sonicated to re-suspend the H-Si NPs. The tubes were then centrifuged at 2,500 $\mathrm{rpm}$ for $10 \mathrm{~min}$ and the supernatant discarded. Finally, the $\mathrm{H}-\mathrm{Si}$ NPs were again re-suspended into a total of $10 \mathrm{~mL}$ of hexane, and immediately studied by FT-IR and PL spectroscopies. 


\subsubsection{Reaction of H-Si NPs with n-hexane}

In order to probe the H-Si NP surface reactivity under mild conditions, H-Si NPs were heated in refluxing, as-received $n$-hexane (b.p. $68^{\circ} \mathrm{C}$ ). A control study was conducted with $n$-hexane that had first been purified to remove unsaturated hydrocarbon impurities (referred to hereafter as purified $n$-hexane). To accomplish these reactions, 10-15 mL of a stock suspension of H-Si NPs was transferred to a 3-neck round bottom flask and the solvent was removed under reduced pressure. Next, the dry H-Si NPs were re-suspended in $3 \mathrm{~mL}$ of $n$-hexane (as-received or purified). Prior to heating, three freeze, pump, thaw cycles were performed to remove atmospheric oxygen. The suspensions were then brought to reflux $\left(68^{\circ} \mathrm{C}\right)$ under flowing Ar for $18-24 \mathrm{~h}$. Upon cooling to room temperature, unreacted $n$-hexane was removed under reduced pressure, and the isolated Si NPs were analyzed by FT-IR and PL spectroscopies.

\subsubsection{Characterization}

Fourier-Transform IR (FT-IR) spectroscopy was conducted using a Thermo Nicolet iS10 spectrophotometer equipped with a diamond, single-bounce attenuated total reflectance (ATR) attachment. Suspensions were measured by depositing drop-cast films onto the ATR attachment and allowing the solvent to evaporate at room temperature. Dry Si NP samples were directly deposited onto the diamond crystal with a disposable plastic spatula and compressed onto the crystal for analysis. A background spectrum was collected prior to each sample spectrum and was subtracted from the sample spectrum using the instrument's software algorithm. In some cases, a linear baseline subtraction was required to correct for light scattering intensity, which increases linearly with energy and results in an apparent decrease in transmittance at higher energies. 
UV-visible absorption spectra were collected using a Shimadzu UV-2450 spectrophotometer. Data were collected from 250 - $700 \mathrm{~nm}$ on diluted suspensions in n-hexane. Photoluminescence emission (PL) and excitation (PLE) measurements were made using a Shimadzu 5301-RFPM spectrophotometer. A diffraction grating monochromator was used to selectively excite the samples with $350 \mathrm{~nm}$ light, which was then passed through a $3 \mathrm{~nm}$ slit prior to entering the sample chamber. Light that was emitted or scattered by the samples was passed through a $5 \mathrm{~nm}$ emission slit prior to reaching the detector. PLE measurements were collected at the maximum emission wavelength of each of the corresponding PL spectra using the same slit widths. Peak intensities were not normalized, but sometimes were magnified to observe weak signals, as indicated.

\subsection{Results and discussion}

During routine chemical analysis of H-Si NPs, strong Si-C bonding at $1260 \mathrm{~cm}^{-1}$ was repeatedly observed by FT-IR, both directly following extraction from the $\mathrm{HF}_{(\mathrm{aq})}$ etch into $n$-hexane, as well as after storage in alkane solvents. We hypothesized that there could be a large number of surface Si radicals present on H-Si NPs, possibly as a result of incomplete hydride passivation by $\mathrm{HF}$, or due to the weak nature of the $\mathrm{Si}-\mathrm{H}$ bond. These highly-reactive surface radicals would be expected to react with alkenes and alkynes, resulting in some degree of HS of the Si NP surface, even under mild conditions. We further hypothesized that the radicals may also be able to react with saturated hydrocarbons to some extent, resulting in partial HS of the Si NP surface. 


\subsubsection{FT-IR characterization of Si NPs reacted with n-hexane}

In order to test our hypotheses, H-Si NPs were heated in refluxing $n$-hexane (b.p. $68^{\circ} \mathrm{C}$ ) under a positive pressure of $\mathrm{Ar}$, and their surface composition was monitored pre- and post-reaction. Figure 3.1 contains FT-IR spectra of the H-Si NP samples before and after heating in refluxing $n$-hexane. Figure 3.1A is a representative FT-IR spectrum of the H-Si NP starting material, either as-prepared or following purification to remove partially oxidized particles prior to heating in refluxing $n$-hexane. Peaks at $2100 \mathrm{~cm}^{-1}$ and $890 \mathrm{~cm}^{-1}$, corresponding to $\mathrm{Si}-\mathrm{H}_{\mathrm{x}}$ stretching and deformation, suggest that the surface composition of the Si NPs prior to reaction is composed of primarily $\mathrm{Si}-\mathrm{H}$ bonding features. ${ }^{78,80,112}$ Furthermore, peaks corresponding to $\mathrm{Si}-\mathrm{O}-\mathrm{X}(\mathrm{X}=\mathrm{Si}, \mathrm{C})$ vibrational modes in the range of $1060-1000 \mathrm{~cm}^{-1}$ are not detectable. ${ }^{13,41,56,62,64,67,80,83,86,89-97,113-116}$ Note that there is a peak at $2340 \mathrm{~cm}^{-1}$ that may be attributed to atmospheric $\mathrm{CO}_{2}$. This feature appears occasionally in FT-IR spectra and is not correlated to the samples.

Significant changes to the surface composition of the Si NPs were observed following their reaction with both as-received $n$-hexane (Figure 3.1B) and $n$-hexane that had first been purified to reduce the concentration of alkene impurities (Figure 3.1C). First, a decrease in the intensity of the $\mathrm{Si}-\mathrm{H}_{\mathrm{x}}$ bonding peaks at 2100 and $890 \mathrm{~cm}^{-1}$ was observed for both products. Second, regardless of the purity of the $n$-hexane, evidence for successful alkyl-passivation of the $\mathrm{Si} \mathrm{NP}$ surface was present for both samples. Specifically, the sharp peak at $1260 \mathrm{~cm}^{-1}(41,67,92,117)$ and the strong, broad peak at $790 \mathrm{~cm}^{-1(67,80,117)}$ found in both Figures 3.1B and 3.1C have been frequently attributed to covalent Si-C bonding. In addition, the peaks observed in Figures 3.1B and $3.1 \mathrm{C}$ at 
$\sim 2900 \mathrm{~cm}^{-1}$ are indicative of $\mathrm{C}-\mathrm{H}_{\mathrm{x}}$ stretching, and their presence is typically attributed to successful HS. ${ }^{64,78,79,118}$

Differences in the FT-IR spectra of the two products suggests that the purity of the $n$-hexane may have had an effect on the extent to which the Si NPs underwent HS. First, the degree of the loss of $\mathrm{Si}-\mathrm{H}_{\mathrm{x}}$ bonding features was varied between the two products, as seen in Figs. 3.1B and 3.1C. After reaction with as-received $n$-hexane, Si- $\mathrm{H}_{\mathrm{x}}$ bonding features were not appreciable in the FT-IR spectrum (Fig. 3.1B), and the C- $\mathrm{H}_{\mathrm{x}}$ bonding features have three intense, discernible peaks at 2960,2925 , and $2850 \mathrm{~cm}^{-1}$. In contrast, following reaction with purified $n$-hexane some intensity was retained in the

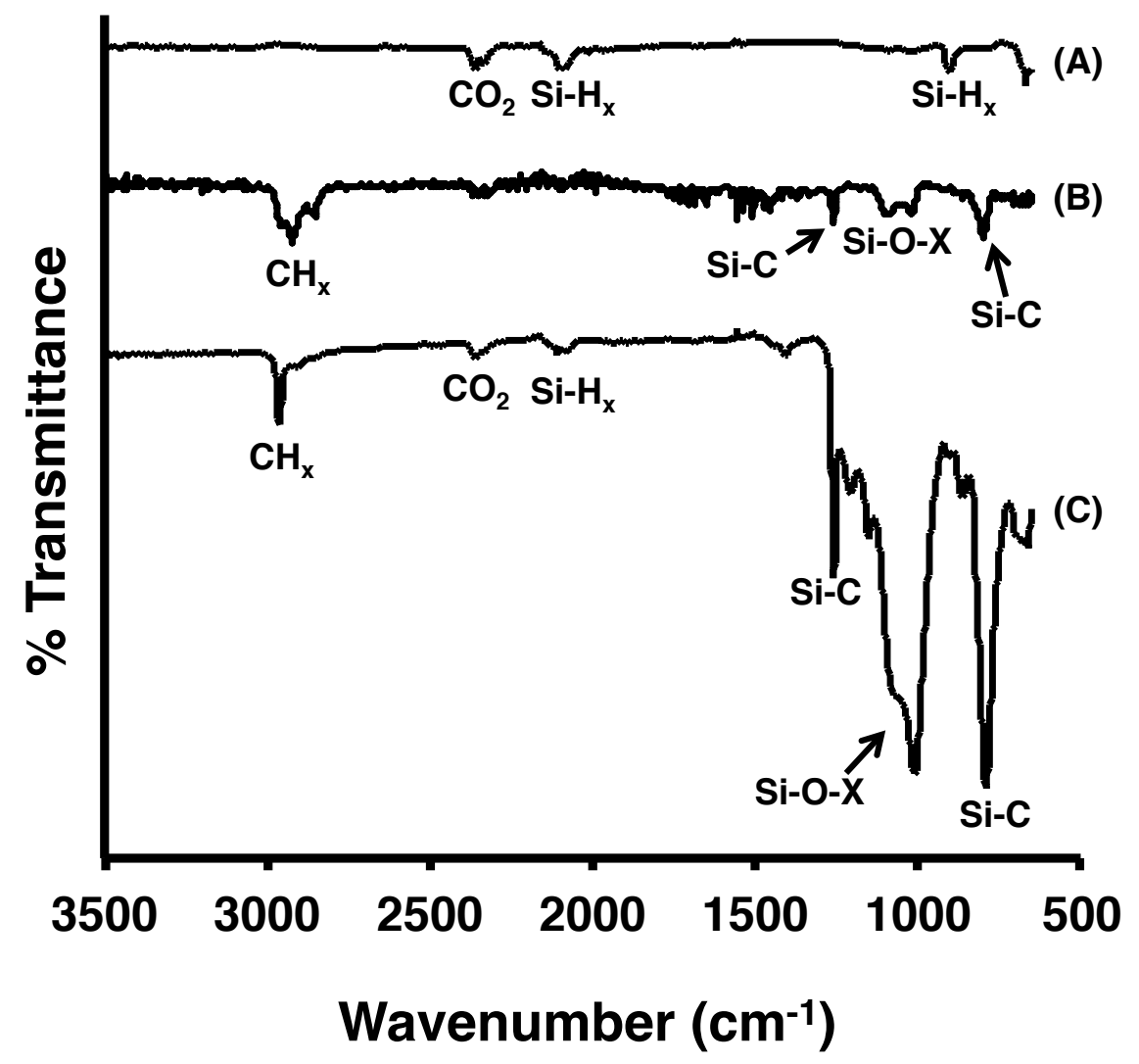

Figure 3.3. FT-IR spectra of (A) freshly-etched H-Si NPs, (B) H-Si NPs following heating in as-received $n$-hexane, and (C) H-Si NPs following heating in purified n-hexane (b.p. $n$-hexane $68^{\circ} \mathrm{C}$ ). 
Si- $\mathrm{H}_{\mathrm{x}}$ bonding region at $\sim 2100 \mathrm{~cm}^{-1}$ (Fig. 3.1C). Furthermore, the $\mathrm{C}-\mathrm{H}_{\mathrm{x}}$ bonding features in Figure 3.1C are dominated by a single, intense peak at $2960 \mathrm{~cm}^{-1}$.

Objectively, these data suggest that purification of the $n$-hexane lowers the extent of Si-C passivation of the Si NP surface. Thus, it is likely that alkene impurities are largely responsible for the observed HS, especially in the case of the H-Si NPs that were heated in refluxing as-received $n$-hexane. If this is the case, we would predict that the HS reaction would proceed according to the mechanism shown in Scheme 3.1. Specifically, Si surface radicals would react with unsaturated bonds in the alkenes, which would initiate the propagation of the reactive radical across the surface of the Si NP. This would account for the observed decrease in $\mathrm{Si}-\mathrm{H}$ bonding on the surface, as this reaction consumes Si-H bonds.

\section{(A)}

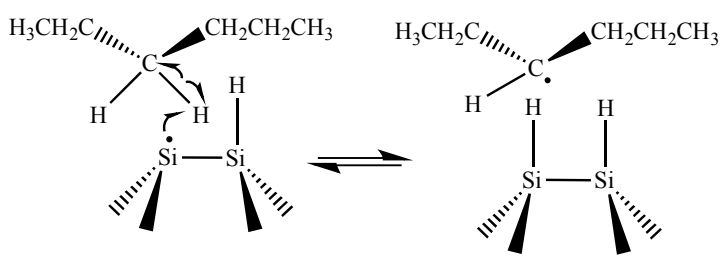

Scheme 3.2. Proposed mechanism for (A) formation of secondary carbon radical by hydride abstraction by an Si radical and (B) coupling of carbon and silicon radicals to form Si-C surface bond.

However, some degree of HS was observed to occur even when $n$-hexane was purified to minimize the presence of alkene impurities. While it is likely that alkene impurities were still present in concentrations that were not detectable by ${ }^{1} \mathrm{H}-\mathrm{NMR}$, we propose an alternate, two-part HS reaction mechanism by which a saturated hydrocarbon may passivate the Si NP surface, as shown in Scheme 3.2. First, a Si surface radical may 
abstract a proton from a saturated hydrocarbon bond to form a secondary carbon radical and an $\mathrm{Si}-\mathrm{H}$ bond (Scheme 3.2A). Next, the unstable carbon radical would be expected to immediately react with either an $\mathrm{Si}-\mathrm{H}$ bond, reforming a surface $\mathrm{Si}$ radical (reverse of Scheme 3.2A). Alternatively, the carbon radical could couple with a surface Si radical, forming an $\mathrm{Si}-\mathrm{C}$ bond as shown in Scheme 3.2B. If this were the case, this reaction would be expected to occur to a greater extent with toluene $v s . n$-hexane, due to the greater electronic stabilization of a resonance stabilized carbon radical vs. a secondary carbon radical.

We submit that the reactions shown in Schemes 3.1 and 3.2 could occur simultaneously, however that reaction with alkenes would be preferred over reaction with $n$-alkanes, due to the low likelihood for forming unstable carbon radicals. However, the Si-C features observed on the surface of both products indicates that some degree of alkylation occurred, even at low temperatures relative to those required to bring commonly-used alkenes to reflux. These data support our hypothesis that radicals are present on the surface of the Si NPs, even in mild conditions. It is reasonable that the radicals may be present either as a result of incomplete surface passivation during the etch or due to some degree of spontaneous homolytic cleavage of the thermodynamically unstable $\mathrm{Si}-\mathrm{H}$ bond at low temperatures. However, it has also been suggested that trace $\mathrm{O}_{2}$ can case $\mathrm{Si}$ radical formation by abstracting a hydride from an H-Si surface. ${ }^{111}$

To this end, oxidation was always observed to occur upon heating, in addition to partial HS of the Si NP surface. This occurred despite the use of air-free techniques and sieve-dried $n$-hexane in both reactions, and even though no surface oxidation was detected on the H-Si NPs by FT-IR spectroscopy prior to reaction. However, a number of 
sources of oxygen are possible such as: 1) incomplete drying of the $n$-hexane by the $4 \AA$ molecular sieves (or from the aluminosilicate sieves themselves), 2) diffusion of oxygen into the reaction flask, 3) the borosilicate glassware or 4) oxidized H-Si NPs not detected by FT-IR prior to reaction. We also note that the peak at $790 \mathrm{~cm}^{-1}$ in the FT-IR spectrum after reaction is located in the region where various $\mathrm{Si}-\mathrm{OH}$ vibrational modes have been noted in the past. ${ }^{119}$ However, the lack of a hydroxyl feature at $3600 \mathrm{~cm}^{-1}$ is inconsistent with the assignment of the peak at $790 \mathrm{~cm}^{-1}$ as due to $\mathrm{Si}-\mathrm{OH}$.

In the FT-IR spectrum of the Si NPs after heating in refluxing as-received $n$-hexane (Fig. 3.1B), there are two peaks of similar intensity at $1000 \mathrm{~cm}^{-1}$ and $1060 \mathrm{~cm}^{-1}$. The appearance of Si-O bonding features is similar in appearance to oxide features that we have previously observed to result from oxidation of the surface by alcohols, ${ }^{67}$ as well as what others have cited as the TO-LO phonon modes of a-SiO $2 .{ }^{96,120}$ In addition, these oxidation-related bonding features are similar in intensity to the vibrational modes corresponding to the alkylation-related bonding features (i.e., Si-C, $\mathrm{CH}_{\mathrm{x}}$ ). Two peaks were also observed in the Si-O bonding region in the FT-IR spectrum of the Si NPs after heating in refluxing purified $n$-hexane (Fig. 3.1C). However, they are found at $995 \mathrm{~cm}^{-1}$ and $1055 \mathrm{~cm}^{-1}$, and the former of the two peaks is significantly greater in intensity than the latter. Moreover, the $1260 \mathrm{~cm}^{-1}(\mathrm{Si}-\mathrm{C})$, and $\mathrm{CH}_{\mathrm{x}}$ bonding features are considerably less intense than the features in the $\mathrm{Si}-\mathrm{O}$ region. Thus, it appears that by decreasing the amount of alkenes in the reaction, HS becomes less favorable, and oxidation of the surface occurs more readily. As the degree of surface oxidation changes, the bonding features in the FT-IR spectra are expected to also change, which we observed to occur. 


\subsubsection{UV-vis characterization of Si NPs reacted with purified n-hexane}

Additionally, UV-visible absorption spectroscopy was used to compare the H-Si NPs before and after heating to reflux in purified $n$-hexane (Figures 3.2A and 3.2B). Both spectra show an absorption onset at roughly $400 \mathrm{~nm}$ with increasing intensity at shorter wavelengths, which is typical of $\mathrm{H}-\mathrm{Si}$ NPs. ${ }^{99,121}$ Two low intensity absorption peaks at $275 \mathrm{~nm}$ and $320 \mathrm{~nm}$ were present in the spectrum of freshly-etched H-Si NPs, shown in Figure 3.2A. The spectrum of Si NPs after reaction with purified $n$-hexane (Figure 3.2B) contains the same feature at $275 \mathrm{~nm}$; however the $320 \mathrm{~nm}$ feature is not discernible.

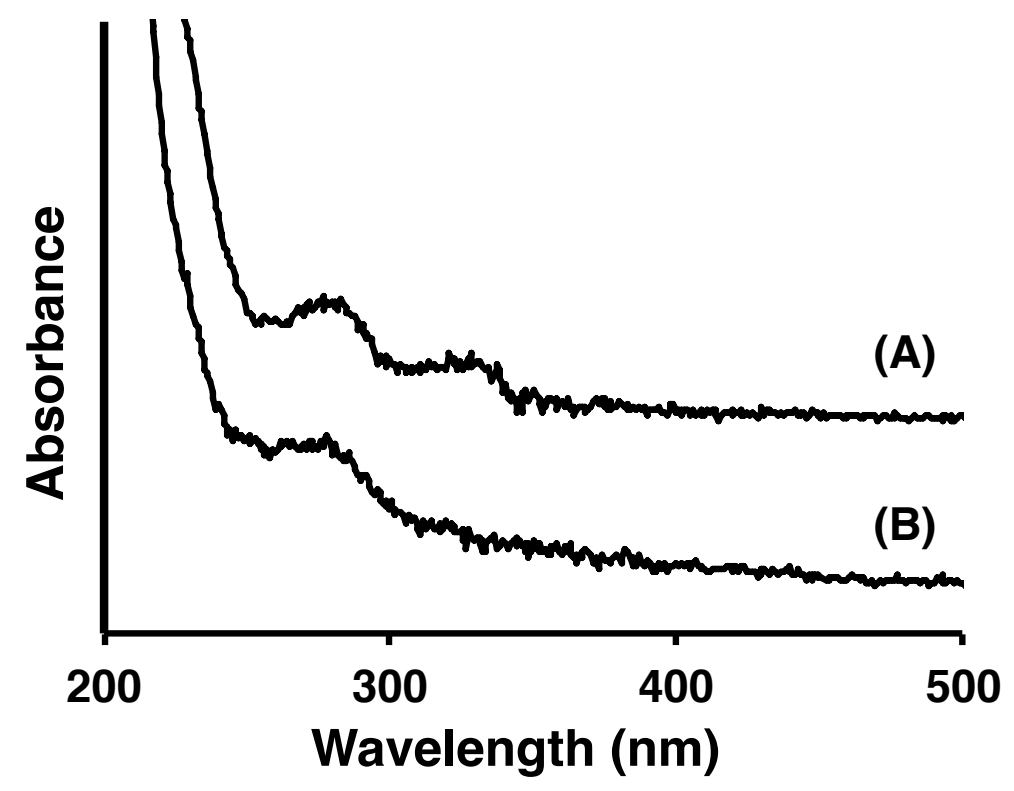

Figure 3.2. UV-visible absorption spectra of (A) H-Si NPs and (B) H-Si NPs following heating in purified $n$-hexane.

\subsubsection{Photoluminescence characterization of Si NPs reacted with $n$-hexane}

To determine the effects of surface reactivity on the optical properties of the H-Si NPs, PL and PLE spectra were collected on the Si NP suspensions before and after 


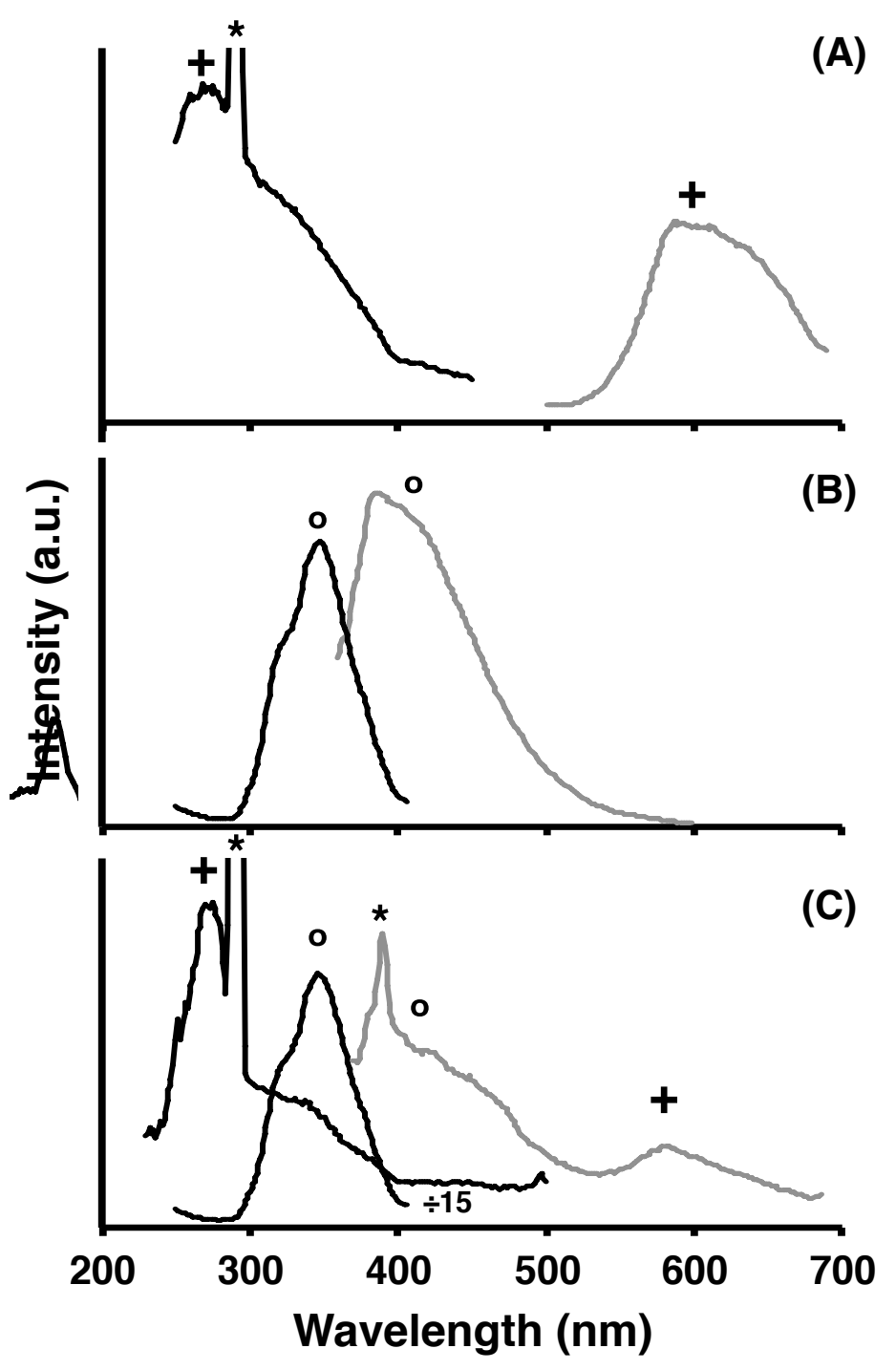

Figure 3.3. PL ( $\lambda_{\mathrm{exc}}=350 \mathrm{~nm}$, gray lines) spectra of (A) H-Si NPs, (B) H-Si NPs heated in as-received $n$-hexane, and (C) H-Si NPs heated in purified $n$-hexane. PLE spectra (black lines) of (A) $\lambda_{\mathrm{em}}=580 \mathrm{~nm}$ (marked with $\mathbf{+}$ ), (B) $\lambda_{\mathrm{em}}=400 \mathrm{~nm}\left(\right.$ marked with $\left.{ }^{\circ}\right)$, and (C) $\lambda_{\mathrm{em}}=580 \mathrm{~nm}$ (marked with +). Light scattering peaks marked with *.

reaction with $n$-hexane. Figure 3.3 contains the PL ( $\lambda_{\mathrm{exc}}=350 \mathrm{~nm}$, gray lines) and PLE spectra (black lines) taken before and after reaction with $n$-hexane. The symbols in the individual panels (A - C) indicate the wavelength in the PL spectrum that was monitored 
in obtaining the corresponding PLE spectrum. In addition, light scattering peaks are indicated with an *, and do not correspond to light emission by the samples.

Typical of minimally oxidized H-Si NPs produced by the method described in Chapter 2, when excited at $350 \mathrm{~nm}$, the prevalent feature in the emission spectrum is a broad, low energy peak (Fig. 3.3A, gray line). ${ }^{23,67}$ Here, the PL spectrum contains a peak with a $\lambda_{\max }$ of $580 \mathrm{~nm}$ with a full-width at half-max (FWHM) of $0.36 \mathrm{eV}$. When monitoring for $580 \mathrm{~nm}$ light emission, the corresponding PLE spectrum (Figure 3.3A, black line) contains a broad peak with a maximum wavelength of $265 \mathrm{~nm}$, and a low energy shoulder that decreases monotonically to approximately $400 \mathrm{~nm}$.

Large differences were observed in the PL spectra of the H-Si NPs following reaction with as-received versus purified $n$-hexane. First, following reaction with as-received $n$-hexane, the PL spectrum is dominated by a higher energy emission (vs. prior to reflux) with maximum intensity at $400 \mathrm{~nm}$ (Fig. 3.3B, gray line). Our group and others have likewise observed the development of strong, high energy PL upon oxidation of originally red-emitting Si NPs. In one study, we reported a red-to-blue PL conversion following oxidation of Si NPs by alcohols. ${ }^{67}$ In another study, blue PL from Si NPs was correlated to trapping of photogenerated charge carriers at defect states corresponding to oxygen and nitrogen impurities. ${ }^{122}$ Alternatively, other groups have attributed blue PL originating from porous silicon (p-Si) to emission from the quantum confined core, while the red emission was attributed to surface oxide ${ }^{66,115}$ However, the changes in surface composition concurrent with the development of blue PL observed in this study points to the former of the explanations: the blue PL results from oxidation of the Si NP surface. 
Although their surfaces were observed to be heavily oxidized by FT-IR spectroscopy, the complete PL conversion that was seen in Figure 3.3B was not observed from the Si NPs that were heated in purified $n$-hexane. The high level of surface oxidation that was present in the FT-IR spectrum of this sample would suggest that the PL spectrum should be dominated by an intense, high energy peak. However, following reaction (Figure 3.3C, gray line), the PL spectrum contains both a low energy emission peak with maximum intensity at $580 \mathrm{~nm}$, and a high energy feature that is overlapped by a Raman scattering peak $(*)$. This suggests that minor differences in the type and extent of surface oxidation, as observed in the FT-IR spectra, may have a large impact on the optical properties of Si NPs.

Changes were also observed in the PLE spectra of the H-Si NPs following reaction with both as-received and purified $n$-hexane. For both products, when monitoring for $400 \mathrm{~nm}$ light emission, the corresponding PLE spectra (Figs. 3.3B and 3.3C, black lines, denoted by symbol $\left({ }^{\circ}\right)$ ) contain a peak that ranges from $300-400 \mathrm{~nm}$, with a maximum wavelength of $345 \mathrm{~nm}$. If the PLE spectrum shown in Figure 3.3A corresponds to interband excitation, then the range of excitation energies that lead to $400 \mathrm{~nm}$ light emission in Figs. 3.3B and 3.3C can be attributed to defect states located between the conduction and valence band extrema. For H-Si NPs reacted with purified $n$-hexane, when monitoring for $580 \mathrm{~nm}$ light emission, the corresponding PLE spectrum (Figure 3.3C, black line, denoted by symbol $(+)$ ) contains a peak that ranges from $260-400 \mathrm{~nm}$, with a maximum excitation wavelength of $265 \mathrm{~nm}$ and a shoulder at $\sim 330 \mathrm{~nm}$, similar to the PLE spectrum in Fig. 3.3A. 


\subsection{Preliminary conclusions}

To summarize, red-emitting H-Si NPs with minimal surface oxidation were heated in refluxing $n$-hexane (b.p. $68^{\circ} \mathrm{C}$ ) to probe the reactivity of the H-Si NP surface with saturated hydrocarbons. An control study was conducted in which $n$-hexane was purified to minimize the concentration of alkene impurities in the reaction, which are known to undergo HS with $\mathrm{H}-\mathrm{Si}$ surfaces. In the studies, Si-C surface bonding was observed by FT-IR spectroscopy following isolation of the Si NPs after reaction. This observation suggests that it is possible to achieve some degree of HS of the H-Si NP surface at low temperatures, implicating the presence of reactive Si-based surface radicals on the H-Si NPs following extraction from the chemical etch. Although we acknowledge that trace alkene impurities could react with the Si-based surface radicals to result in partial HS, we provided an alternative explanation to account for our observations. We propose that the Si radicals react with $n$-hexane, according to Scheme 3.2, resulting in the observed Si-C bonding.

In addition, the development of $\mathrm{Si}-\mathrm{O}$ bonding features along with a reduction in the original Si-H bonding features was observed for both samples; however the latter occurred to a greater extent when a higher concentration of alkene impurities were likely present in the $n$-hexane. When $\mathrm{Si}-\mathrm{H}$ surface bonding was observed by FT-IR spectroscopy, red PL was observed in addition to a high energy PL feature. When no Si-H bonding was detected, only blue PL was observed. The data presented herein strongly suggest that H-Si NPs are highly unstable, and prone to oxidation. Thus, if the surface is not idealized, defects can dominate the absorption and PL decay pathways, as we further examine in Chapter 4. 


\section{Chapter 4}

Investigation into the effect of (2,2,6,6-tetramethyl-piperidin-1-yl)oxyl radical on silicon nanoparticle photoluminescence

\subsection{Introduction}

The stability of photoluminescence (PL) from visibly red-emitting, hydridepassivated silicon nanoparticles (H-Si NPs) in the presence of a radical trap was probed. Upon addition of (2,2,6,6,-tetramethyl-piperidin-1-yl)oxyl radical (TEMPO), the energy and intensity of what has previously been assigned as an interband transition was observed to change over time, dependent on the reaction conditions. First, when the reaction occurred at $4^{\circ} \mathrm{C}$ with minimal light exposure, the interband transition was observed to shift somewhat gradually, in a manner similar to what has previously been attributed to core shrinking and quantum confinement. Second, when the reaction occurred at room temperature with exposure to $360 \mathrm{~nm}$ irradiation at regular intervals, the interband transition was observed to be quenched as a new transition at $575 \mathrm{~nm}$ developed. In the latter study, the low energy PL was determined to undergo oxidative quenching by TEMPO. Finally, as a result of chemical analysis of the Si NP surfaces before and after reaction with TEMPO, we propose that extensive, photo-induced surface oxidation is the cause of the intense $575 \mathrm{~nm}$ emission.

\subsection{Motivation for the present study}

Based on past experimental and theoretical work, it can be concluded that the stability of interband transitions in Si NPs is highly dependent on the stability of the Si NP surface. ${ }^{51,54,55,57-65,123,124}$ These studies have frequently linked changes in surface 
composition to the development of electronically active (defect) states within the bandgap. If the defect states that form are radiative recombination centers, the Si NP suspension would be expected to exhibit new photoluminescence (PL) bands. For instance, we have previously observed the development of PL bands owing to surface oxidation by alcohols. ${ }^{67}$ However, if the defect states that form are non-radiative recombination centers, the Si NP suspension would be expected to exhibit interband PL with reduced intensity. Si-based radicals, for example, are one class of defects that have been commonly reported to result in decreased intensity of interband nanocrystalline Si PL.

Indeed, for free-standing, colloidal Si NPs, one of the most commonly cited sources of non-radiative decay is the silicon dangling bond trap state. ${ }^{13,49,57,65,67,72,125-128}$ The presence of $\mathrm{Si}$ surface radicals on $\mathrm{Si}$ NPs has been supported by electron paramagnetic resonance (EPR) spectroscopy, and various causes for their existence have been suggested. Two common causes that have been suggested are the formation of lowquality or disordered surface oxides, yielding EPR-active $\mathrm{SiO}_{2}-\mathrm{Si} \bullet,{ }^{56,72}$ and desorption of hydrogen from the H-Si NP surface, which yields EPR-active $\mathrm{Si}_{3}-\mathrm{Si} \bullet .{ }^{49,56,74}$ Moreover, several groups have suggested that hydrosilylation (HS) of the $\mathrm{Si}-\mathrm{H}$ surface is initiated by the homolytic cleavage of Si-H surface bonds, thereby forming Si surface radicals. ${ }^{83-}$ $86,81,87$

Experimentally, a reduction in the concentration of dangling bonds in Si NP samples has been correlated to increased PL intensity. For example, in one study, red-emitting $\left(\lambda_{\text {em }}=730 \mathrm{~nm}, \lambda_{\text {exc }}=325 \mathrm{~nm}\right)$ Si NPs embedded in $\mathrm{SiO}_{2}$ were annealed at $1100^{\circ} \mathrm{C}$ in forming gas $\left(95 \% \mathrm{~N}_{2}+5 \% \mathrm{H}_{2}\right)$ in order to promote hydride-passivation of $\mathrm{Si}$ 
dangling bond sites. ${ }^{72}$ The annealing procedure was found to result in a reduction in the EPR signal intensity, which was correlated with increased PL intensity. In another study, thin films of Si NPs were subjected to HF-etching followed by annealing at $200^{\circ} \mathrm{C}$ under vacuum. ${ }^{14} \mathrm{~A}$ tenfold decrease in the EPR signal was observed, which was correlated with a decrease in sub-bandgap light absorptivity, as well as an increase in the conductivity of the films. In short, both of these studies provided evidence that showed that hydridepassivation of Si radical sites can result in increased PL intensity.

Although hydride-passivation of Si radicals may be one way of increasing the yield of interband transitions, as we showed in Chapter 3, H-Si NP surfaces are unstable. Even under mild conditions relative to standard HS reactions, the Si NP surfaces were shown to undergo partial alkylation, even in $n$-hexane solvent. Furthermore, changes in the surface composition were accompanied by substantial changes in the optical properties of the suspensions. Specifically, new PL bands were observed spectroscopically, which occurred alongside changes to the surface composition. Ultimately, these observations led us to propose that mechanisms involving highlyreactive Si radicals could account for the changing PL and surface composition over time.

Consequently, if radicals remain, or are formed in situ, on the surfaces of the Si NPs, then it is reasonable to expect the surface composition of the Si NPs to change as a function of time and chemical environment. In addition, the Si NP suspensions would likewise be expected to exhibit unstable PL, both in the energy and intensity of the transition. Thus, one way to suppress dynamic surface reactions, and therefore stabilize the PL, would be to passivate radicals that are presumably present on the Si NP surface. TEMPO, or (2,2,6,6-tetramethyl-piperidin-1-yl)oxyl), has a stable nitroxy radical and has 
previously been shown to sequester Si radicals both on the surface of hydride-passivated planar Si and p-Si surfaces, ${ }^{129-136}$ as well as to sequester Si radicals in polysilanes. ${ }^{137-140}$ With this in mind, we hypothesized that TEMPO should also be able to sequester $\mathrm{Si}$ radicals on the surfaces of $\mathrm{H}-\mathrm{Si} \mathrm{NPs}$, which would be expected to both increase PL intensity and slow or prevent further reactivity and changes in PL energy.

Here we consider two probable causes for the presence of Si surface radicals on the H-Si NPs studied in Chapter 3. First, it is possible that some concentration of radicals are present as a result of incomplete passivation of the surface during the chemical etch. In this case, we would expect TEMPO to passivate the existing radicals, which would result in an increase in the Si NP PL as well as a stabilization of the PL energy. Second, it is possible that homolytic cleavage of the $\mathrm{Si}-\mathrm{H}$ bond may occur at mildly elevated temperatures due to the low thermodynamic stability of the Si-H bond relative to other surface bonding (e.g., Si-C, Si-O). In this case, where the radicals form in situ, we would expect TEMPO to fully react with the Si NP surface until all of the Si-H bonds have been replaced. As a result, the PL would be expected to exhibit gradual changes until the surface replacement has been completed.

The study presented herein examines the interaction between H-Si NPs and TEMPO by PL spectroscopy, in order to monitor changes to the Si NP PL as a result of the addition of the radical trap to the suspension. Analysis of the surface composition was additionally performed to monitor for chemical changes that may have been associated with the observed changes to the Si NP PL. 


\subsection{Experimental}

\subsubsection{Materials}

Trichlorosilane $\left(\mathrm{HSiCl}_{3}, 98 \%\right.$, Ar-protected, Alfa Aesar, Ward Hill, MA), absolute ethanol (abs-EtOH, $\mathrm{C}_{2} \mathrm{H}_{5} \mathrm{OH}$, anhydrous, Pharmco-AAPER, Brookfield, CT), ethanol (EtOH, ACS grade, stockroom), hydrofluoric acid (HF, ACS reagent, 48-51\% solution in water, Acros Organics, NJ), hexane $\left(\mathrm{C}_{6} \mathrm{H}_{14},+95 \%\right.$, Sigma-Aldrich, St. Louis, MO), toluene (reagent grade, stockroom), and (2,2,6,6-tetramethyl-piperidin-1-yl)oxyl (TEMPO, $\mathrm{C}_{9} \mathrm{H}_{18} \mathrm{NO}, 98 \%$, Acros Organics, NJ) were purchased and used as received unless otherwise specified. Millipore water $(18 \mathrm{M} \Omega \bullet \mathrm{cm}$ resistivity) was used during the polycondensation and etching procedures. Laboratory deionized water $\left(\mathrm{DI} \mathrm{H}_{2} \mathrm{O}\right.$ ) was used during the purification of hexane.

\subsubsection{Preparation of $\mathrm{H}-\mathrm{Si} \mathrm{NPS}$}

H-Si NPs were produced according to a previously published protocol, ${ }^{23}$ which has been discussed in detail in Chapter 2. Briefly, $\left(\mathrm{HSiO}_{1.5}\right)_{\mathrm{n}}$ was prepared through polycondensation and polymerization of $\mathrm{HSiCl}_{3}$ with $\mathrm{H}_{2} \mathrm{O}$, and dried at $100^{\circ} \mathrm{C}$ under flowing Ar. Approximately $1.0 \mathrm{~g}$ of the resulting white polymer was loaded into a $5 \mathrm{~mL}$ alumina combustion boat and then annealed at $1100^{\circ} \mathrm{C}$ for $10 \mathrm{~h}$ under flowing $\mathrm{N}_{2}$. The resulting product was a light brown powder that exhibited red PL under irradiation with $365 \mathrm{~nm}$ light directly following annealing. Next, $0.45-0.55 \mathrm{~g}$ of the annealed product was added to a PTFE sample cup equipped with a magnetic stir bar, to which $5 \mathrm{~mL}$ of abs-EtOH and $5 \mathrm{~mL}$ of $\mathrm{H}_{2} \mathrm{O}$ was added, and the solid was dispersed by sonication. Then, $5 \mathrm{~mL}$ of HF was added and the mixture was left to stir loosely covered for $60 \mathrm{~min}$. The $\mathrm{H}-\mathrm{Si}$ NPs liberated from the oxidized matrix were then phase-transferred into $40 \mathrm{~mL}$ of 
either hexane or toluene, and stored under Ar until further use. Samples were studied by FT-IR and PL spectroscopies prior to subsequent surface-reactivity studies.

\subsubsection{Preparation of H-Si NP/TEMPO suspensions}

Stock suspensions of H-Si NPs in either hexane or toluene were prepared according to the method described in Chapter 2. For each study, sample aliquots were removed from a stock suspension. The initial (and final) Si NP sample concentrations in the aliquots derived from the stocks in the following studies were not determined, but were assumed to be the same for each sample within a set, since one stock suspension was used per study. The maximum TEMPO concentration used in the studies was determined based on a calculation to estimate the number of surface $\mathrm{Si}$-atoms present in $5 \mathrm{~mL}$ of a stock suspension, based on the assumptions that $145 \mathrm{mg}$ of $5 \mathrm{~nm} \mathrm{Si} \mathrm{NPs}$ were present. Equation 4.1 summarizes the method used to approximate the number of surface Si atoms present in $5 \mathrm{~mL}$ of a stock suspension.

$$
N_{\text {total }}^{\text {Si atoms }} \approx \frac{V_{\text {shell }}}{V_{u c}} \times N_{u c \text { face }}^{\text {Si atoms }} \times N_{\text {total }}^{\text {Si NPs }}
$$

Equation 4.1

In the above equation, $V_{\text {shell }} 0.54 \mathrm{~nm}$ thick shell for a sphere with a diameter of $5 \mathrm{~nm}$ and $V_{u c}$ is the volume of a single $\mathrm{Si}$ unit cell $(\mathrm{a}=0.54 \mathrm{~nm})$. Thus, the ratio of the two terms approximates the number of unit cells present on the surface of a $5 \mathrm{~nm}$ Si NP. That number is then multiplied by the total number of Si atoms on a single face of a unit cell $(4$ corner atoms, 1 face atom $=1$ total $)$ to determine the number of surface $\mathrm{Si}$ atoms on a single $5 \mathrm{~nm}$ Si NP. Finally, that number is then multiplied by the number of Si NPs estimated to be present in $5 \mathrm{~mL}$ of a stock solution. $N_{\text {total }}^{S i N P S}$ was estimated by multiplying the density of Si by volume of a $5 \mathrm{~nm} \mathrm{Si} \mathrm{NP}$, and then dividing that mass by the average mass of Si NPs isolated from the stock suspension. The total number of Si 
atoms was estimated to be $\sim 10^{20}$, which corresponds to $\sim 0.003$ moles of surface Si atoms (n(surface $\mathrm{Si}))$ in solution.

In the first study, the reaction between H-Si NPs and TEMPO was analyzed over a range of TEMPO concentrations with respect to time (up to one week). Sample preparation was conducted as follows. First, between 0.00 and $11.40 \mathrm{mg}$ of solid TEMPO was added to six $15 \mathrm{~mL}$ scintillation vials. The vials were then sealed with rubber septa and purged with Ar. A stock solution of H-Si NPs in toluene was divided equally into the vials, resulting in final TEMPO concentrations of $0.00,0.192,0.969,2.13,3.83$, and $10.4 \mathrm{mM}$. Each vial was subsequently sonicated for a maximum of 10 seconds to ensure mixing, and then kept at $4^{\circ} \mathrm{C}$ in the dark, except for when aliquots were removed for analysis. Aliquots of each sample were removed from the vials for characterization by PL spectroscopy after one, two and seven days. Qualitative observations of sample PL were made by irradiation of the samples with a handheld $365 \mathrm{~nm}$ UV-lamp; digital images were taken for comparison.

To further examine the initial interaction between the H-Si NPs and TEMPO (i.e., at earlier time points), another study was conducted in which the samples were monitored every $10 \mathrm{~min}$ by PL spectroscopy immediately following sample preparation. Sample preparation was conducted as follows. First, between 0.00 and $37.43 \mathrm{mg}$ of solid TEMPO was added to five $15 \mathrm{~mL}$ scintillation vials, followed by the addition of a $5 \mathrm{~mL}$ aliquot of a stock solution of H-Si NPs in hexane to each vial. The total suspension volume was brought to $15 \mathrm{~mL}$ by addition of toluene (dried over $4 \AA$ molecular sieves), resulting in approximate final TEMPO concentrations of $0.0,0.5,2.0,8.0$ and $16.0 \mathrm{mM}$. The five samples were prepared over a span of five days (one per day), and the stock 
suspension was stored under $\operatorname{Ar}$ at $4^{\circ} \mathrm{C}$ in between sample preparations. After each suspension was prepared, it was left to stir under flowing Ar for 10 min to ensure proper mixing. An aliquot of each suspension was subsequently removed from the vial $10 \mathrm{~min}$ after mixing and characterized by PL spectroscopy over the course of the next $24 \mathrm{~h}$.

\subsubsection{Scale-up of 8.0 mM TEMPO sample for chemical analysis}

To afford sufficient amounts of sample for chemical analysis by infrared (IR) and X-ray photoelectron spectroscopies (XPS), another room temperature stored, mid-range TEMPO concentration sample with a total volume of $30 \mathrm{~mL}$ was prepared. First, $34.52 \mathrm{mg}$ of TEMPO was added to a round bottom flask, to which $20 \mathrm{~mL}$ of dry toluene was added. Next, $10 \mathrm{~mL}$ of a stock suspension of H-Si NPs in hexane was added, which resulted in a final TEMPO concentration of $8 \mathrm{mM}$. Following mixing, the sample was left to stir at room temperature under flowing Ar and continuous illumination by ambient light. After 24 and $48 \mathrm{~h}$, an aliquot of the sample was analyzed by PL spectroscopy, and the remainder of the suspension was purified to remove excess TEMPO from the sample. Post-reaction purification was conducted as follows: the suspension was split equally amongst two glass centrifuge tubes, and centrifuged at 2,000 rpm for $10 \mathrm{~min}$. The supernatant was decanted, and $10 \mathrm{~mL}$ of hexane was added to each tube, which were then sonicated to re-disperse the Si NPs and any remaining TEMPO. The samples were then centrifuged again at 2,000 rpm for $20 \mathrm{~min}$ and the supernatant again decanted. Finally, the isolated solid was analyzed by FT-IR spectroscopy.

\subsubsection{Photoluminescence characterization of Si NP/TEMPO suspensions}

Steady-state photoluminescence (PL) spectroscopy was conducted on the samples from the first study using a PTI QuantaMaster 300 spectrophotometer equipped with a 
pulsed Xe laser source. PL samples for these measurements were prepared by drawing aliquots from the sample vials after 1,2, and 7 days, and spectra were acquired in ambient air immediately after the aliquots were brought to RT. During each aliquot extraction, care was taken to avoid the introduction of $\mathrm{O}_{2}$ to the remaining sample. PL measurements of the aliquots were done in ambient air immediately following extraction from the vials in order to minimize oxidation of the Si NPs. Spectra were recorded from 350 to $730 \mathrm{~nm}$, and the excitation wavelength of $370 \mathrm{~nm}$ was chosen to eliminate interference of the second order scattering peak of the excitation beam $\left(\lambda_{\max }=740 \mathrm{~nm}\right)$ from the sample PL.

Due to instrument accessibility and availability, long-duration PL spectroscopy experiments were conducted on the samples from the second study using a Shimadzu 5301-RFPM spectrophotometer equipped with a Xe laser source. Spectra were recorded from 450 to $800 \mathrm{~nm}$ with an excitation wavelength of $360 \mathrm{~nm}$ due to the higher absorption cross section of the suspension at 360 in comparison to $370 \mathrm{~nm}$. A band pass filter $\left(340-385 \mathrm{~nm}, \lambda_{\max }=363 \mathrm{~nm}\right)$ was inserted following the excitation slit to eliminate interference of the second order diffraction of the excitation beam $\left(\lambda_{\max }=726 \mathrm{~nm}\right)$. In addition, a $450 \mathrm{~nm}$ long pass filter was inserted prior to the emission slit to allow for the use of a wider excitation slit to increase the intensities of all PL events. This filter was used to absorb sub-450 nm light due to high intensity emission or particle light scattering, which would overwhelm the interband PL under investigation. PL samples for these measurements were prepared by removing $2.5 \mathrm{~mL}$ aliquots from the suspensions and injecting them into an air-free quartz cuvette. The cuvette was then sealed with a rubber septum and purged with Ar for 10 min prior to analysis. PL spectra 
were collected every $10 \mathrm{~min}$ for a total of $24 \mathrm{~h}$, with the cuvette remaining in the sample chamber for the duration of the study. The cuvettes were agitated once after $\sim 16 \mathrm{~h}$ to re-suspend Si NPs that had precipitated out of solution. Additional PL spectra of the samples from the second study were collected over the spectral range $390-700 \mathrm{~nm}$ $\left(\lambda_{\text {exc }}=360 \mathrm{~nm}\right)$ at $\mathrm{t}=0 \mathrm{~h}$ and $24 \mathrm{~h}$ without the addition of the filter set in order to observe the full Si NP PL spectrum.

\subsubsection{Chemical analysis of large-scale $8.0 \mathrm{mM}$ TEMPO sample}

Fourier-Transform infrared (FT-IR) spectroscopy was conducted using a Thermo Nicolet iS10 spectrometer equipped with a diamond, single-bounce attenuated total reflectance (ATR) attachment. Suspensions were measured by depositing drop-cast films onto the ATR attachment and allowing the solvent to evaporate at room temperature. A reference spectrum of TEMPO was also collected by directly depositing the solid onto the diamond crystal with a disposable plastic spatula; the solid was compressed onto the crystal for measurement. A background spectrum was collected prior to each sample spectrum and was subtracted from the sample spectrum using the instrument's software algorithm.

\subsection{Results and discussion}

As part of our previously published study, ${ }^{67}$ an experiment was conducted in which varying amounts of TEMPO were added to three vials containing red-emitting H-Si NP suspensions, which were taken from a single stock solution. The intensity of the red emission was observed by PL spectroscopy to increase upon addition of TEMPO to the suspensions, relative to the intensity of the control sample to which no TEMPO was 
added (see Appendix, Figure A.1). We hypothesized that the initial increase in PL intensity, the extent of which was observed to be dependent on the [TEMPO], was due to the elimination of silicon radicals on the surfaces of the Si NPs upon coupling with the TEMPO oxygen radicals. We further hypothesized that TEMPO would continue to passivate Si surface radicals if they were formed in situ (i.e., by homolytic $\mathrm{Si}-\mathrm{H}$ bond cleavage), resulting in the eventual stabilization of the PL energy and intensity.

\subsubsection{Reaction of H-Si NPs with TEMPO: effect of time on PL (study one)}

In order to test our hypothesis regarding the long-term stabilization of the Si NP surface and PL, suspensions of H-Si NPs and TEMPO were prepared and their PL was analyzed over the course of a week. First, red-emitting $\left(\lambda_{\mathrm{exc}}=370 \mathrm{~nm}, \lambda_{\mathrm{em}}=700 \mathrm{~nm}\right)$ suspensions of H-Si NPs in toluene were added to five vials containing between 0.00 and $11.40 \mathrm{mg}$ of TEMPO to result in TEMPO concentrations between $0.00 \mathrm{mM}$ and 10.4 mM, as summarized in Table 4.1. The concentrations of Si NPs were not measured, but were assumed to be roughly equivalent in the aliquots, since the aliquots were taken from a single stock suspension of H-Si NPs. Following preparation, the samples were stored under a blanket of $\mathrm{Ar}$, in the dark, at $4^{\circ} \mathrm{C}$. Aliquots were removed at $\mathrm{t}=1,2$, and $7 \mathrm{~d}$ for analysis by PL spectroscopy.

Table 4.1. Sample preparation parameters for first variable TEMPO concentration study.

\begin{tabular}{cccc}
\hline Sample & $\begin{array}{c}\text { Mass of TEMPO } \\
\text { (mg) }\end{array}$ & $\begin{array}{c}\text { [TEMPO] } \\
(\mathbf{m M})\end{array}$ & $\begin{array}{c}\text { n(TEMPO) / } \\
\text { n(surface Si) }\end{array}$ \\
\hline I & 0.000 & 0.000 & - \\
II & 0.302 & 0.129 & $6.5 \mathrm{E}-4$ \\
III & 2.271 & 0.969 & $4.8 \mathrm{E}-3$ \\
IV & 4.992 & 2.13 & $1.1 \mathrm{E}-2$ \\
V & 8.977 & 3.83 & $1.9 \mathrm{E}-2$ \\
VI & 24.375 & 10.4 & $5.2 \mathrm{E}-2$ \\
\hline
\end{tabular}


Figure 4.1 contains digital images of the Si NP suspensions 5 min (Fig. 4.1A) and $90 \mathrm{~min}$ (Fig. 4.1B) following sample preparation. After $5 \mathrm{~min}$, the red PL appeared visibly quenched for samples II - VI vs. the control (I). The extent of quenching was observed to be greater the greater the amount of TEMPO added to the suspension (Fig. 4.1A). Due to the relatively long excited state lifetime of interband transitions in indirect bandgap semiconductors ( $\mu$ s to $\mathrm{ms}$ ), Si NPs are highly susceptible to carrier trapping and PL quenching by other faster processes. ${ }^{49,50,5}$ It is therefore possible that the observed initial decrease in interband PL intensity is due to either electron or energy transfer involving photo-excited Si NPs and TEMPO.
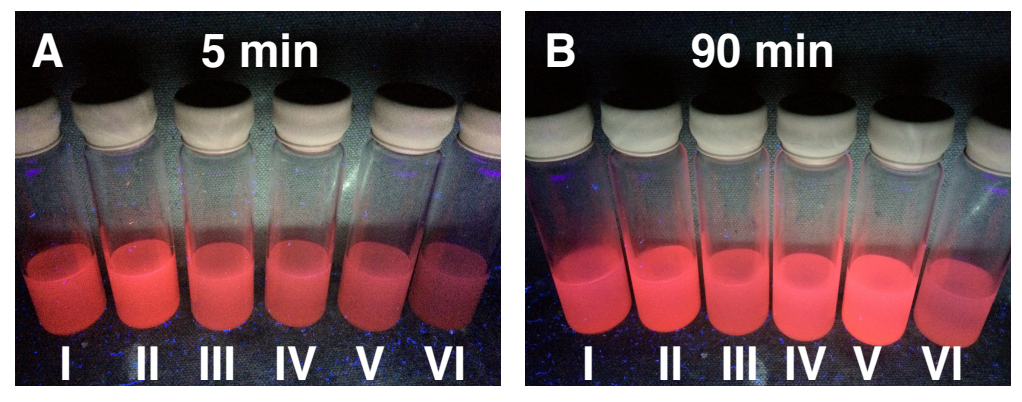

Figure 4.1. Digital images showing visible PL from H-Si NPs in toluene (A) 5 min and (B) $90 \mathrm{~min}$ after addition of TEMPO to the suspensions $\left(\lambda_{\mathrm{exc}}=365 \mathrm{~nm}\right)$. The samples from left to right contain $0.00 \mathrm{mM}$ (I), $0.129 \mathrm{mM}$ (II), 0.969 (III), $2.13 \mathrm{mM}$ (IV), $3.83 \mathrm{mM}$ (V), and $10.4 \mathrm{mM}$ TEMPO (VI).

However, 90 min after sample preparation there was a visible increase in the red PL intensity for samples II - VI (Figure 4.1B) relative to what was observed 5 min after adding TEMPO to the suspensions. This visual observation is similar to what we have previously observed spectroscopically (see Figure A.1). ${ }^{67}$ In light of the implication of radicals on the surfaces of $\mathrm{H}-\mathrm{Si}$ NPs from the studies in Chapter 3, we propose that TEMPO may trap Si surface radicals by coupling with the TEMPO radical. Since Si radicals are often proposed to be the source of non-radiative trap states, ${ }^{13,49,57,65,67,72,125-128}$ 
these observations support our hypothesis that trapping of the Si surface radicals results in an increase in the intensity of interband PL from H-Si NPs.

However, at longer time points, large changes in the energy and intensity of the Si NP PL were observed spectroscopically. Figure 4.2 contains the PL spectra of samples I - VI taken $1 \mathrm{~d}$ (black lines), $2 \mathrm{~d}$ (red lines), and $7 \mathrm{~d}$ (blue lines) after sample preparation. For all of the samples, with the exception of VI containing the highest

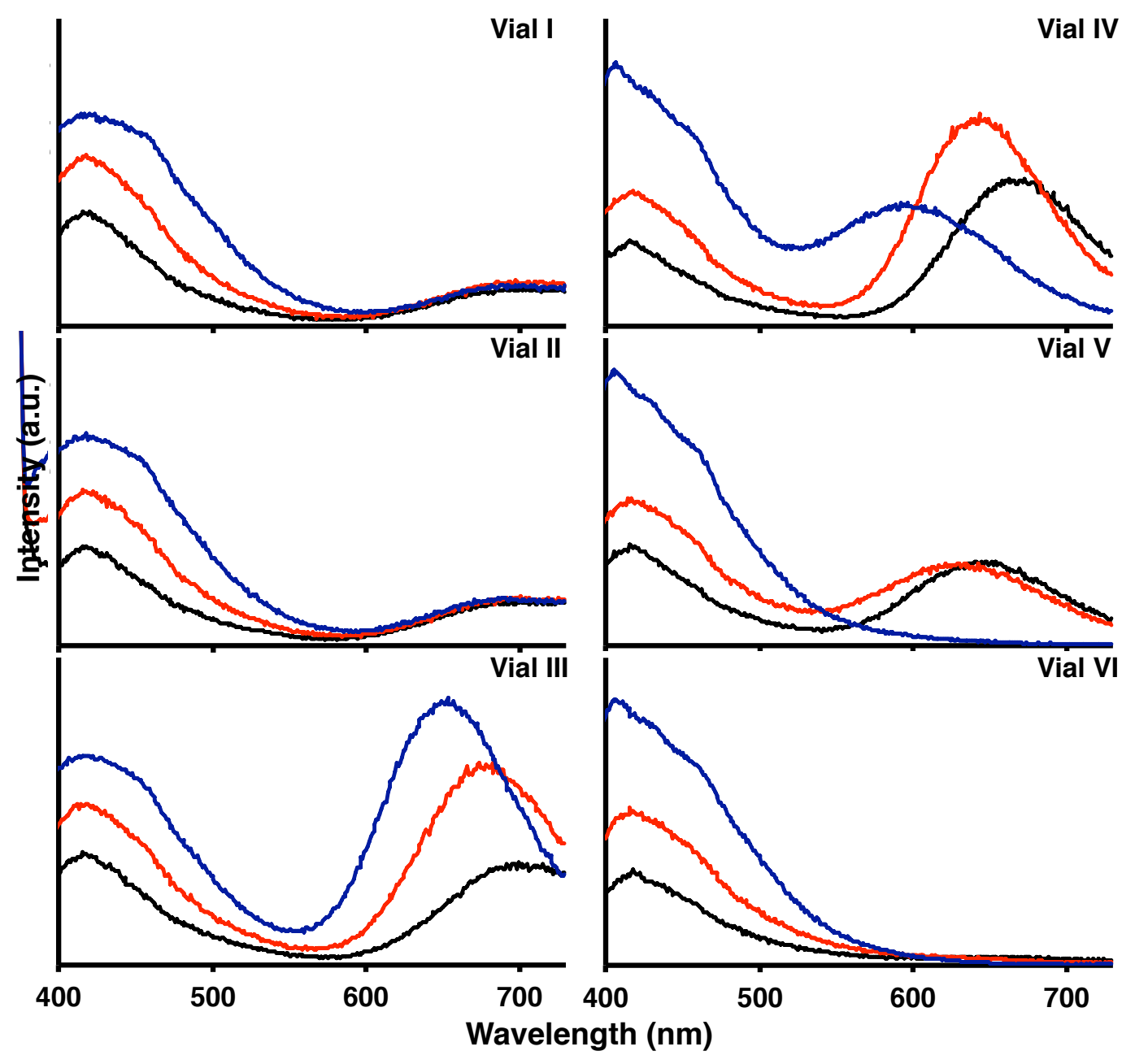

Figure 4.2. PL spectra of Si NPs in sample vials I - VI of study 1, containing variable [TEMPO]: 1 day (black lines), 2 days (red lines) and 7 days (blue lines) following addition of TEMPO to the suspensions $\left(\lambda_{\mathrm{exc}}=370 \mathrm{~nm}\right)$. The concentrations of TEMPO in the samples are $0.00 \mathrm{mM}$ (I), $0.129 \mathrm{mM}$ (II), 0.969 (III), $2.13 \mathrm{mM}$ (IV), $3.83 \mathrm{mM}$ (V), and $10.4 \mathrm{mM}$ (VI). 
[TEMPO], both a low energy emission peak with a $\lambda_{\max }$ between 650 and $700 \mathrm{~nm}$ and a high energy emission peak with a $\lambda_{\max }$ of $415 \mathrm{~nm}$ were observed one day after sample preparation (black lines); only the high energy emission peak is observed for sample VI. For all of the samples (II - VI), the high energy emission peak was observed to increase in intensity over time. The same observation was made for the control sample (I), and the presence of the high energy emission peak was therefore determined to be independent of the presence of TEMPO. We have previously observed dual $450 \mathrm{~nm}$ and $580 \mathrm{~nm}$ emissions from alkyl-passivated Si NPs $\left(\lambda_{\text {exc }}=370 \mathrm{~nm}\right)$, wherein we attributed the $450 \mathrm{~nm}$ emission to a defect state resulting from oxidation of the Si NP surface. ${ }^{67}$ Similarly, Veinot and coworkers have attributed the development of a $\sim 450 \mathrm{~nm}$ emission $\left(\lambda_{\text {exc }}=370 \mathrm{~nm}\right)$ to trace nitrogen and oxygen impurities in Si NPs produced via a similar method. ${ }^{63}$ In these cases, an increase in the intensity of the high energy emission over time would be consistent with an increase in oxidation of the H-Si NP surface.

Changes to the low energy emission were also observed to occur over time, but unlike the high energy emission, were observed to be dependent on the addition of TEMPO to the samples. First, below a threshold concentration of TEMPO (samples I and II), no change in the energy and intensity of the low energy $\left(\lambda_{\max }=700 \mathrm{~nm}\right)$ emission was observed to occur over the course of the seven days that the samples were characterized. Therefore, at all time points, the PL spectra of samples I and II were dominated by the high energy $\left(\lambda_{\max }=415 \mathrm{~nm}\right)$ emission. It is possible that at low concentrations of TEMPO, such as was the case for sample II, not enough TEMPO was present to elicit a drastic change in the average PL intensity of the Si NPs in the suspension. Second, above a threshold concentration of TEMPO (sample VI), no 
intensity was observed in the low energy spectral region at any of the time points analyzed in the study. Finally, for the samples containing the mid-range of TEMPO concentrations (samples III $-\mathbf{V}$ ), the low energy emission was observed to gradually change in energy over time.

Figure 4.3 is a plot of the maximum emission wavelength of the low energy emission versus time for samples III (red line), IV (green line), and V (violet line). For all three mid-range [TEMPO] samples, the low-energy PL band was observed to shift towards higher energies with time. In addition, the magnitude of this shift, relative to the control sample $\left(\lambda_{\max }=700 \mathrm{~nm}\right)$, at any given time point was greater for samples containing higher concentrations of TEMPO. As can be seen in Figure 4.3, at $\mathrm{t}=1 \mathrm{~d}$ the $\lambda_{\max }$ of the low energy emission from sample III was $700 \mathrm{~nm}$, similar to samples I and II. In contrast, the $\lambda_{\max }$ of the low energy emission from samples $\mathbf{I V}$ and $\mathbf{V}$ had already

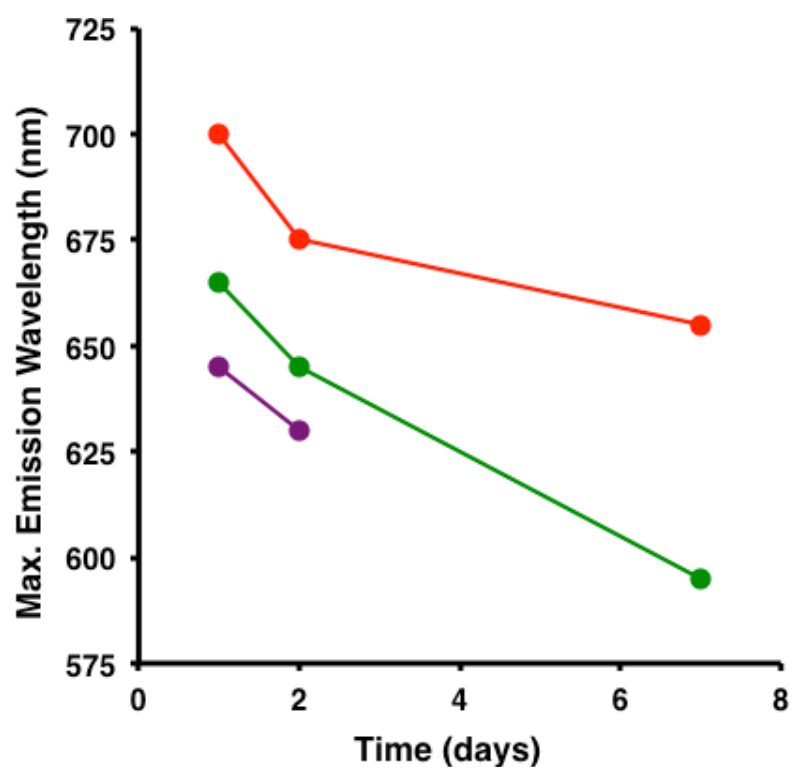

Figure 4.3. Plot of maximum emission wavelength versus time for samples III (red), IV (green), and V (violet). 
shifted to $670 \mathrm{~nm}$ and $650 \mathrm{~nm}$, respectively. Further hypsochromic shifting of the $\lambda_{\max }$ of the low energy emission was observed $2 \mathrm{~d}$ after sample preparation for all three of the samples, and all three continued to shift to higher energy with increased time. Seven days after sample preparation, the $\lambda_{\max }$ of the low energy emission was measured for samples III and IV at $655 \mathrm{~nm}$ and $595 \mathrm{~nm}$, respectively. However, the low energy emission was no longer observed from $\mathbf{V}$ at the same time point.

Other groups have reported similar shifts in interband PL, where a hypsochromic shift in the observed PL corresponded to a reduction in the H-Si NP core diameter and is consistent with quantum confinement. ${ }^{56,21}$ In these studies, a chemical etch is typically used to produce size-selected H-Si NPs with tunable interband PL energies. If the $700 \mathrm{~nm}$ emission that was observed prior to the addition of TEMPO is indeed due to recombination of charge carriers across the bandgap, then it is likely that the hysopchromic shift in emission energy we observe may be due to core shrinkage. Since the same shift was not observed for the $\lambda_{\max }$ of the low energy emission from the control sample, we propose that TEMPO is likely responsible for the observation.

In addition to the changes in energy, the intensity of the low energy emission was also observed to change over time for samples III, IV, and $\mathbf{V}$ (Figure 4.2). Both one day and two days after sample preparation (black and red lines, respectively), the PL spectra of samples III, IV, and $\mathbf{V}$ all exhibited increases in the intensity of the low energy emission relative to the control sample (I). However, while an increase in intensity was observed again for sample III seven days after sample preparation, both IV and V exhibited a decrease in the intensity of the low energy emission relative to two days after. The magnitude of the decrease in intensity from $\mathbf{V}$ seven days after sample preparation 
was large enough such that the low energy emission was completely quenched. Furthermore, the visual observations described below suggest that given enough time, the low energy emission will either exhibit a hypsochromic shift large enough to become indistinguishable from the high energy emission peak, or be completely quenched by other processes, for all samples independent of the presence TEMPO.
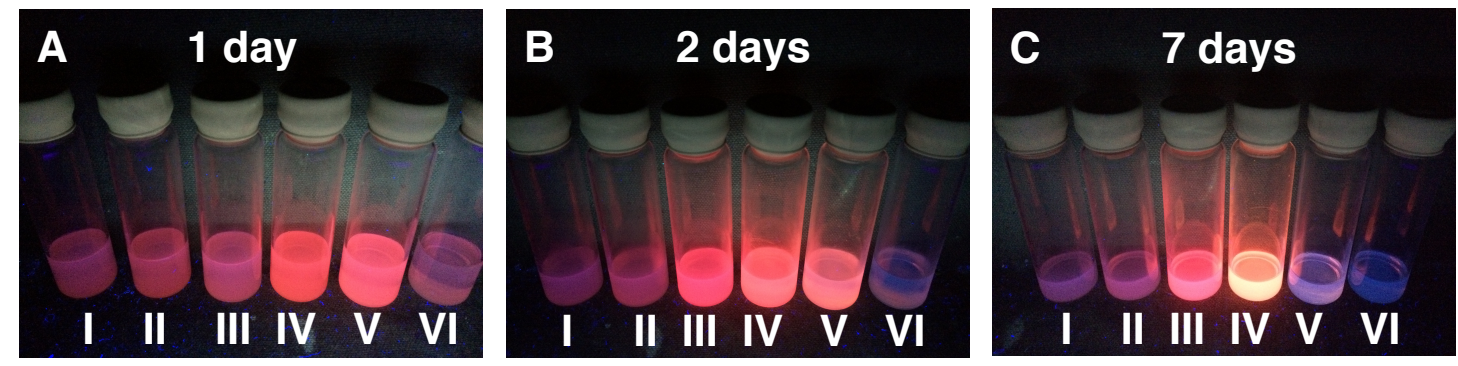

Figure 4.4. Digital images showing visible PL from H-Si NPs in toluene/TEMPO solutions (A) 1 day, (B) 2 days, and (C) 7 days following addition of TEMPO to the suspensions $\left(\lambda_{\text {exc }}=365 \mathrm{~nm}\right)$. The samples from left to right contain $0.00 \mathrm{mM}$ (I), $0.129 \mathrm{mM}$ (II), 0.969 (III), $2.13 \mathrm{mM}$ (IV), $3.83 \mathrm{mM}$ (V), and $10.4 \mathrm{mM}$ TEMPO (VI).

Spectroscopic data correlate well with visual observation of the samples over time. Figure 4.4A-C show photographs of the same samples under $365 \mathrm{~nm}$ illumination taken in addition to the spectroscopic measurements described above. Relative to the samples at the time points shown in Figure 4.1, samples I - VI (except for IV) appear to have taken on a a pink hue. This visual observation is supported spectroscopically, where the intensity of the high energy emission was greater than the intensity of the low energy emission. Note that samples III and IV, which were observed spectroscopically to have the most intense low energy emission peaks at two and seven days after sample preparation, appear visibly the brightest in Figs. 4.4B and 4.4C.

TEMPO has previously been shown to effectively passivate Si-based radicals on planar Si and as well as within polysilanes. ${ }^{129,131,133-139,141}$ Therefore, we began this work 
with the proposal that TEMPO would also effectively passivate Si based radicals on the surfaces of Si NPs. However, homolytic cleavage of the relatively weak Si-H surface bond could produce more Si surface radicals over time. If TEMPO is present then it is possible that it could continue to passivate the Si NP surface. It should be mentioned that in one recent study, formation of Si surface radicals was proposed to occur by cleavage of $\mathrm{Si}_{-} \mathrm{SiH}_{3}$ surface bonds rather than cleavage of $\mathrm{Si}-\mathrm{H}$ surface bonds. ${ }^{82}$ The authors argued that the $\mathrm{Si}-\mathrm{Si}$ bond, which is slightly weaker than the $\mathrm{Si}-\mathrm{H}$ bond $(\sim 75 \mathrm{kcal} / \mathrm{mol} v s$. $\sim 90 \mathrm{kcal} / \mathrm{mol}$, respectively), was more likely to undergo dissociation to produce $\mathrm{Si}$ surface radicals. Loss of $\bullet \mathrm{SiH}_{3}$ from the surface would be expected to result in a decrease in the Si NP diameter, alongside diminished PL intensity due to the formation of a corresponding Si radical. However, Si surface radicals formed over time due to the loss of $\cdot \mathrm{SiH}_{3}$ could also be passivated by TEMPO, and the net effect of radical formation and quenching by this mechanism would also be expected to be interband PL with increased energy.

Passivation of Si surface radicals by TEMPO, however, could also affect the local energy structure of the Si atom that it binds to. Specifically, the oxidation state of the surface-bound $\mathrm{Si}$ atom would be expected to increase upon $\mathrm{Si}-\mathrm{O}$ bond formation. Previously, oxidation of the Si NP surface has been shown to be an effective method for reducing the core diameter of Si NPs. For example, in one study, ${ }^{125}$ Si NP size reduction resulted in a hypsochromic shift in the Si NP PL, corresponding to an increase in the bandgap energy due to QC. Thus, in light of all the data shown in Figs. 4.1 - 4.4, we propose that oxidation of the Si NP surface by TEMPO, possibly in combination with 
loss of $\bullet \mathrm{SiH}_{3}$ from the surface, is consistent with the [TEMPO]-dependent hypsochromic shift in the $\lambda_{\max }$ of the low energy PL transition.

\subsubsection{Reaction of H-Si NPs with TEMPO: closer look at initial brightening (study two)}

A separate spectroscopic investigation of the first $24 \mathrm{~h}$ period following addition of TEMPO to Si NP suspensions was conducted in order to examine in greater detail the initial intensity fluctuations that occurred for the low energy emission at the shortest time points ( $\mathrm{t}<1 \mathrm{~d}$, see Figures 4.1 and A.1). Unlike the high energy peak, the low energy peak was observed to be sensitive to the concentration of TEMPO.

In the second study, a stock suspension of H-Si NPs in hexane was stored under Ar at $4^{\circ} \mathrm{C}$ in the dark in between sample preparations to minimize sample reactivity. Each sample was prepared immediately prior to analysis, such that the second study spanned six days in total. Samples were prepared according to the follow methods. First, $5.0 \mathrm{~mL}$ aliquots of red-emitting $\left(\lambda_{\mathrm{exc}}=360 \mathrm{~nm}, \lambda_{\mathrm{em}}=660 \mathrm{~nm}\right)$ suspensions of H-Si NPs in hexane were added to five vials containing between 0.000 and $37.500 \mathrm{mg}$ of TEMPO, and diluted to $15.0 \mathrm{~mL}$ with toluene to result in TEMPO concentrations between

Table 4.2. Sample preparation parameters for second variable TEMPO concentration study.

\begin{tabular}{cccc}
\hline Sample & $\begin{array}{c}\text { Mass of TEMPO } \\
(\mathbf{m g})\end{array}$ & $\begin{array}{c}\text { [TEMPO] } \\
(\mathbf{m M})\end{array}$ & $\begin{array}{c}\mathbf{n} \text { (TEMPO) / } \\
\mathbf{n} \text { (surface Si) }\end{array}$ \\
\hline $\mathbf{1}$ & 0.000 & 0.000 & - \\
$\mathbf{2}$ & 1.172 & 0.500 & $2.5 \mathrm{E}-3$ \\
$\mathbf{3}$ & 4.688 & 2.00 & $1.0 \mathrm{E}-2$ \\
$\mathbf{4}$ & 18.750 & 8.00 & $4.0 \mathrm{E}-2$ \\
$\mathbf{5}$ & 37.500 & 16.0 & $8.0 \mathrm{E}-2$ \\
\hline
\end{tabular}


$0.000 \mathrm{mM}$ and $16.0 \mathrm{mM}$, as summarized in Table 4.2. Next, an aliquot of each sample was removed 10 minutes after mixing and added to an Ar-filled cuvette sealed with a rubber septum. PL measurements were made every 10 minutes for a total of 24 hours, and the samples were left in the instrument in between measurements.

Similar to what was observed visually in Figure 4.1, the $660 \mathrm{~nm}$ PL that was initially present for the control sample was partially quenched upon addition of TEMPO

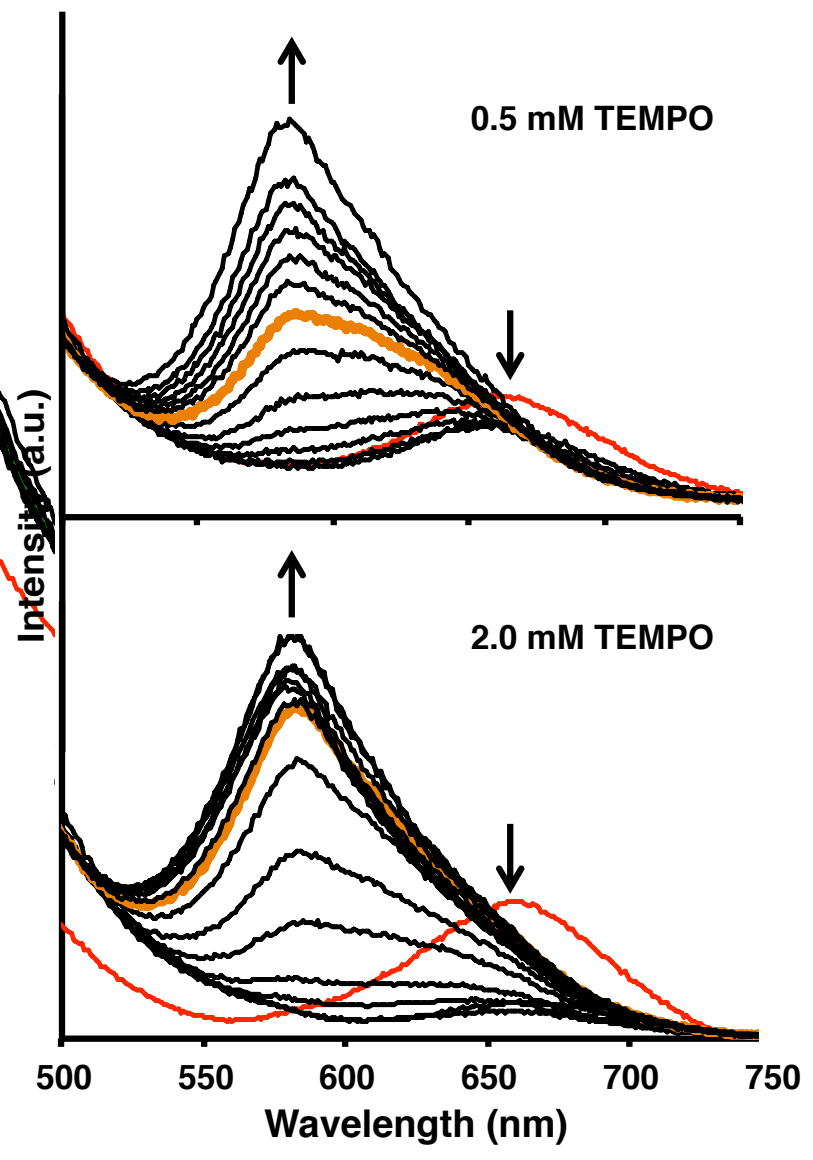

Figure 4.5. PL spectra $\left(\lambda_{\text {exc }}=360 \mathrm{~nm}\right)$ of H-Si NPs in (top) $0.5 \mathrm{mM}$ TEMPO and (bottom) $2.0 \mathrm{mM}$ TEMPO from $\mathrm{t}=0 \mathrm{~h}-4.7 \mathrm{~h}$ in $20 \mathrm{~min}$ intervals. The PL spectrum of the control with no TEMPO added from $\mathrm{t}=0 \mathrm{~h}$ is shown in red, and the PL of the samples at $\mathrm{t}=2.3 \mathrm{~h}$ is shown in orange. Arrows highlight changes in intensity of the two peaks shown in each panel. 
to the suspensions. As can be seen in Figure 4.5, upon addition of TEMPO, the low energy emission is initially lower in intensity relative to the control sample at $\mathrm{t}=0 \mathrm{~h}$ (red lines). In addition, the magnitude of quenching was greater for the $2.0 \mathrm{mM}$ TEMPO sample (bottom panel) than for the $0.5 \mathrm{mM}$ TEMPO sample (top panel).

To further characterize the PL quenching mechanism, the ratio of the unquenched PL intensity to the quenched PL intensity, or quenching efficiency, was plotted versus concentration of TEMPO (Figure 4.6). The resulting linear relationship can be fit by the Stern-Volmer equation:

$$
\frac{I_{o}}{I}=K_{S v}[T E M P O]+1
$$

where $I_{0} / I$ is the quenching efficiency and $K_{s v}\left(2.3373 \mathrm{M}^{-1}\right)$ is the quenching constant, extracted from the slope of the trendline in Figure 4.6. The results of the Stern-Volmer analysis suggest that the PL quenching occurs via transfer of an electron to (from) TEMPO from (to) a photo-excited Si NP. Similar dynamic quenching mechanisms have previously been observed for a variety of nanocrystalline Si systems. ${ }^{37,38,142-144}$ For example, oxidative quenching of alkyl-passivated photo-excited Si NPs by nitroaromatic compounds has been shown to be an efficient process, and has furthermore been exploited in the development of molecular sensors. ${ }^{142}$

Similar to the first study presented herein, the $\lambda_{\max }$ of the low energy emission was also observed spectroscopically to change over time in this study. However, unlike the first study, a gradual hypsochromic shift was not observed for the low energy emission from the partially quenched samples. Rather, the $660 \mathrm{~nm}$ PL band that was initially observed from the H-Si NP suspensions was replaced by an intense $575 \mathrm{~nm}$ emission over time. This trend was observed for all samples, including the control, which 


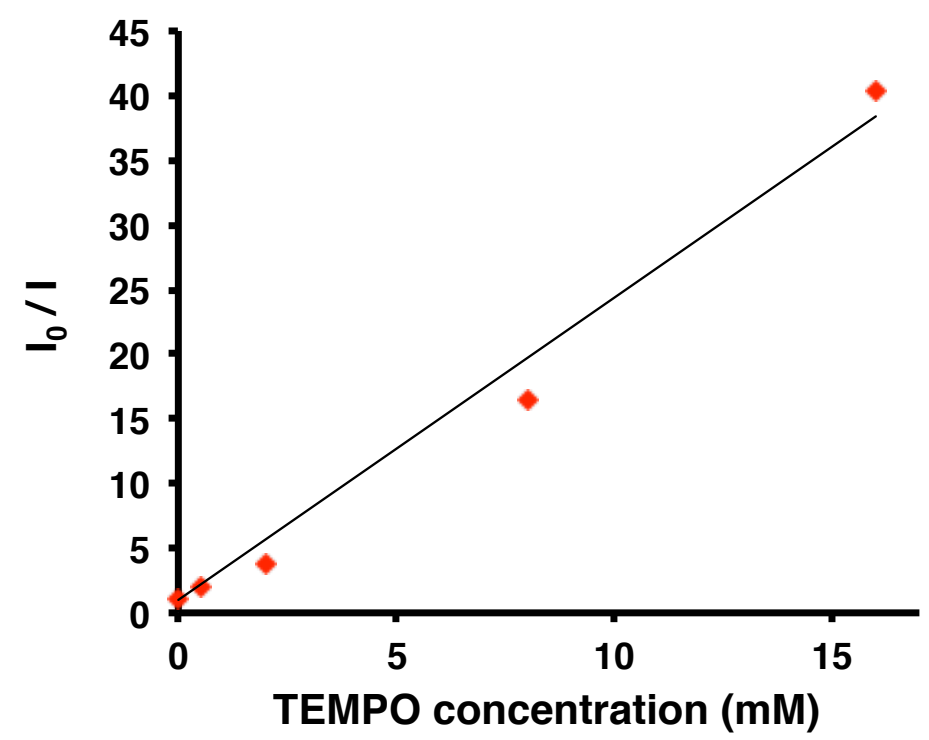

Figure 4.6. Stern-Volmer analysis of quenching of $660 \mathrm{~nm}$ PL by TEMPO.

suggests that the new PL band at $575 \mathrm{~nm}$ does not result from interaction of the Si NPs with TEMPO. Finally, it should be noted that for the sample containing the highest concentration of TEMPO $(16.0 \mathrm{mM})$, no appreciable intensity was ever observed in the low energy region of the PL spectrum following addition of TEMPO. This is consistent with the observation made in the previous study for sample VI $(10.4 \mathrm{mM})$; both observations could be due to extensive quenching of interband PL by at relatively high [TEMPO] in solution.

The rate of the PL conversion from $660 \mathrm{~nm}$ to $575 \mathrm{~nm}$ was observed to be dependent on the concentration of TEMPO, wherein samples with higher concentrations of TEMPO exhibited faster PL conversions. For example, comparison of the PL spectra of the $0.5 \mathrm{mM}$ and $2.0 \mathrm{mM}$ TEMPO samples 2.3 hours after sample preparation (Fig. 4.5, orange lines) illustrates the difference in the rate of PL conversion as a function of 
TEMPO concentration. Specifically, the PL spectrum of the $0.5 \mathrm{mM}$ TEMPO sample remains highly asymmetric, with intensity at the original $660 \mathrm{~nm}$ PL peak still contributing significantly to the overall PL spectrum. However, the PL spectrum of the 2.0 $\mathrm{mM}$ TEMPO sample at the same time point is dominated fully by the $575 \mathrm{~nm}$ emission. For samples containing even higher concentrations of TEMPO, the low energy PL conversion was even more rapid.

The second study was intended to carefully monitor the changes in sample PL that were visually observed in the first study immediately following addition of TEMPO to the H-Si NPs. However, as a result of frequent spectrum acquisition, the samples were irradiated with $360 \mathrm{~nm}$ light once every ten minutes over the course of one day. Therefore, it is not surprising that the observed changes to sample PL were different in this study vs. the former. The results of Stern-Volmer analysis of the quenched $660 \mathrm{~nm}$ emission suggest that TEMPO quenches photo-excited Si NPs via an electron transfer mechanism, likely by oxidation of the photo-excited Si NP cores. Concerning the $575 \mathrm{~nm}$ transition, previously, size-independent low energy PL has been observed from nanocrystalline $\mathrm{Si}$ systems, particularly porous $\mathrm{Si}(\mathrm{p}-\mathrm{Si}) .{ }^{52,115,69}$ In each of these studies, the observed low energy emission was attributed to radiative recombination of charge carriers at an interfacial layer between the crystalline silicon cores and a surface oxide.

To monitor for the sub-450 nm PL that was observed in the first study, the entire spectral range ( $390 \mathrm{~nm}$ to $700 \mathrm{~nm}$ ) was measured for all of the samples both immediately following preparation $(t=0 \mathrm{~h})$ and 24 hours following sample preparation. Figure 4.7 contains the PL spectra (colored lines) of all samples taken at $\mathrm{t}=0 \mathrm{~h}$ (top) and $\mathrm{t}=24 \mathrm{~h}$ (bottom). Note that light scattering peaks, which do not correspond to sample PL, 
are indicated with $(*)$. For all samples, a high energy emission feature with a $\lambda_{\max }$ of $420 \mathrm{~nm}(390-500 \mathrm{~nm})$ was observed immediately following sample preparation (Figure 4.6, top panel). This emission peak has the same $\lambda_{\max }$ as the high energy emission peak observed in the study described in Section 4.4.1, and we therefore tentatively assign it as the same surface-oxide related defect. In addition, for the samples containing less than or equal to $2.0 \mathrm{mM}$ TEMPO, the $660 \mathrm{~nm}$ emission was also present in the PL spectrum. We have previously provided support for this transition as due to interband radiative recombination, ${ }^{67}$ which is also consistent with the hypsochromic shift of the low energy PL band in the first study. Finally, as was observed in Figure 4.5, 24 hours following sample preparation, the $660 \mathrm{~nm}$ emission was replaced by a $575 \mathrm{~nm}$ emission for all samples that were subjected to frequent UV irradiation. However, 24 hours following sample preparation, all samples have retained the high energy feature, which would be consistent with a persisting oxide species.

The PLE spectra of the observed emissions were also measured immediately after and one day following sample preparation (black and gray lines). When monitoring for $420 \mathrm{~nm}$ intensity, at both time points the PLE spectrum (gray trace) contains two distinguishable peaks ranging from $275 \mathrm{~nm}-390 \mathrm{~nm}$ (local maxima of $305 \mathrm{~nm}$ and $345 \mathrm{~nm}$ ). No significant changes were observed in the PLE spectra of the high energy PL feature between $0 \mathrm{~h}$ and $24 \mathrm{~h}$ (gray lines, top and bottom panels). At both time points, the $345 \mathrm{~nm}$ peak in each spectrum is more intense than the $305 \mathrm{~nm}$ peak. In contrast, there were significant differences in the energies observed to excite the low energy emissions at $\mathrm{t}=0 \mathrm{~h}$ and $\mathrm{t}=24 \mathrm{~h}$ (black lines, top and bottom panels). When monitoring for the $660 \mathrm{~nm}$ emission observed at $\mathrm{t}=0 \mathrm{~h}$, the PLE spectrum contains an intense peak ranging 
from $275 \mathrm{~nm}-320 \mathrm{~nm}\left(\lambda_{\max }=290 \mathrm{~nm}\right)$, with a low intensity shoulder that decreases in intensity to $400 \mathrm{~nm}\left(\lambda_{\max }=340 \mathrm{~nm}\right)$. However, when monitoring for the $575 \mathrm{~nm}$ emission observed at $\mathrm{t}=24 \mathrm{~h}$, the PLE spectrum looks much more similar to the PLE spectrum corresponding to the $420 \mathrm{~nm}$ emission. Specifically, the spectrum contains two overlapping peaks ranging from $275-400 \mathrm{~nm}$, with $\lambda_{\max }$ of $305 \mathrm{~nm}$ and $345 \mathrm{~nm}$.

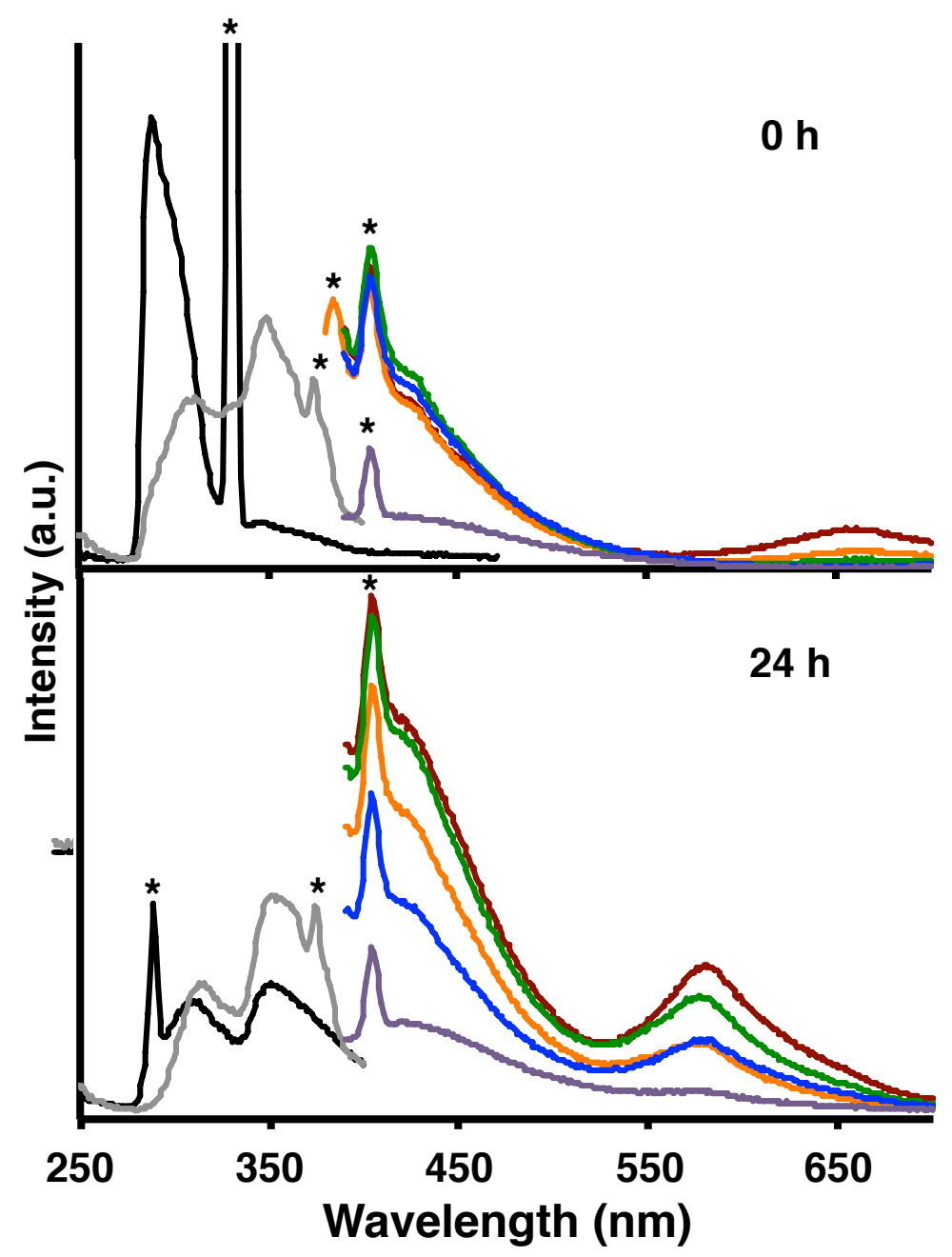

Figure 4.7. PL Spectra of the control (red), as well as H-Si NPs in $0.5 \mathrm{mM}$ (orange), $2.0 \mathrm{mM}$ (green), $8.0 \mathrm{mM}$ (blue), and $16.0 \mathrm{mM}$ (violet) TEMPO, taken at (top) $0 \mathrm{~h}$ and (bottom) $24 \mathrm{~h}$. PLE spectra shown in black correspond to the emission intensity monitored at $650 \mathrm{~nm}$ (top) and $575 \mathrm{~nm}$ (bottom). The PLE spectra shown in gray correspond to the PL feature monitored at $420 \mathrm{~nm}$ (both panels). Light scattering peaks are indicated by * or have been eliminated from the spectra for clarity. 
However, unlike the PLE spectrum corresponding to $420 \mathrm{~nm}$ intensity, the two peaks are relatively similar in intensity.

\subsubsection{Chemical analysis of $8.0 \mathrm{mM}$ TEMPO sample}

In order to be able to isolate sufficient quantities of Si NPs for further chemical analysis, an additional study was done in which an $8.0 \mathrm{mM}$ TEMPO sample containing Si NPs was repeated at double the volume in the previous study. In the scale-up study, the suspension was stirred continuously under flowing Ar and standard overhead lighting for 48 hours prior to isolation of the Si NPs for FT-IR analysis. Aliquots of the sample were removed at $t=24 \mathrm{~h}$ and $\mathrm{t}=48 \mathrm{~h}$ for characterization of the sample PL to ensure the same spectroscopic changes had occurred (shown in Figure A.2).

Figure 4.9 contains the FT-IR spectra of (A) freshly-prepared H-Si NPs, (B) solid TEMPO and (C) Si NPs isolated from 8.0 mM TEMPO solution. Prior to reaction with TEMPO, the FT-IR spectrum of the Si NPs suggests that their surfaces are largely $\mathrm{H}$-passivated, as indicated by the strong $\mathrm{Si}-\mathrm{H}$ bonding and deformation peaks at $2100 \mathrm{~cm}^{-1}$ and $890 \mathrm{~cm}^{-1}$, respectively. In addition, there is a weak, broad peak present at $1050 \mathrm{~cm}^{-1}$, which is the region where Si-O bonding is typically observed. Following isolation from the reaction with $8.0 \mathrm{mM}$ TEMPO, very little of the initial Si-H surface bonding was retained in the FT-IR spectrum (Fig. 4.8C). Furthermore, the extent of surface oxidation of the Si NP surface has drastically increased relative to what was observed for the H-Si NPs. Specifically, there are four peaks at 1188, 1143, 1029 and $844 \mathrm{~cm}^{-1}$, none of which were detected in Figure 4.8A.

In this study, toluene was used as either a solvent or co-solvent to increase the stability of the Si NP suspensions. If the conclusions in Chapter 3 are correct, then we 
would expect that toluene would more readily passivate the Si NP surface by the mechanism shown in Scheme 3.2, due to the resonance stabilization of the carbon radical formed in Scheme 3.2A. In this work, we note the absence of sharp peaks where we would expect to see $\mathrm{Si}-\mathrm{C}$ vibrational modes in the FT-IR spectrum of the Si NPs following reaction with TEMPO (i.e., 1260 and $790 \mathrm{~cm}^{-1}$ ). TEMPO has been shown to prevent hydrosilylation of planar $\mathrm{H}-\mathrm{Si}$ surfaces with alkenes by coupling to the $\mathrm{Si}$ radicals. ${ }^{129,131,132}$ Therefore, it is plausible that TEMPO would likewise prevent alkylation of Si NP surfaces.

Finally, the FT-IR spectrum of the Si NPs following reaction with TEMPO bears little resemblance to the reference spectrum of solid TEMPO (Fig. 4.8B). This suggests that either TEMPO did not passivate the surface, or is present below the limit of detection of the spectrometer. One possible explanation for the absence of TEMPO features in the FT-IR spectrum of the isolated Si NPs can be found in similar studies in which Si radicals in polysilanes were trapped with TEMPO. Specifically, authors have reported evidence for the desorption of TEMPO from Si to occur by preferential cleavage of the N-O bond over the Si-O passivation bonding. ${ }^{137-139}$ This was shown to occur only when additional $\mathrm{Si}$ radicals were near the $\mathrm{Si}$ atom bound to TEMPO. In one study, the authors reported that the Si-O radical formed by $\mathrm{N}-\mathrm{O}$ bond dissociation could further react with a vicinal Si-Si bond. ${ }^{139}$ In addition, the $\mathrm{N}$ radical formed by N-O bond dissociation could further react with an $\mathrm{Si}-\mathrm{H}$ bond in the polysilane to form an amine and regenerate a Si radical.

As a result of the changes in surface composition of the Si NPs, we propose that the observed $575 \mathrm{~nm}$ PL is due to UV-induced development of defects at the interface between crystalline $\mathrm{Si}$ and the surface oxide. Similar observations have been previously 


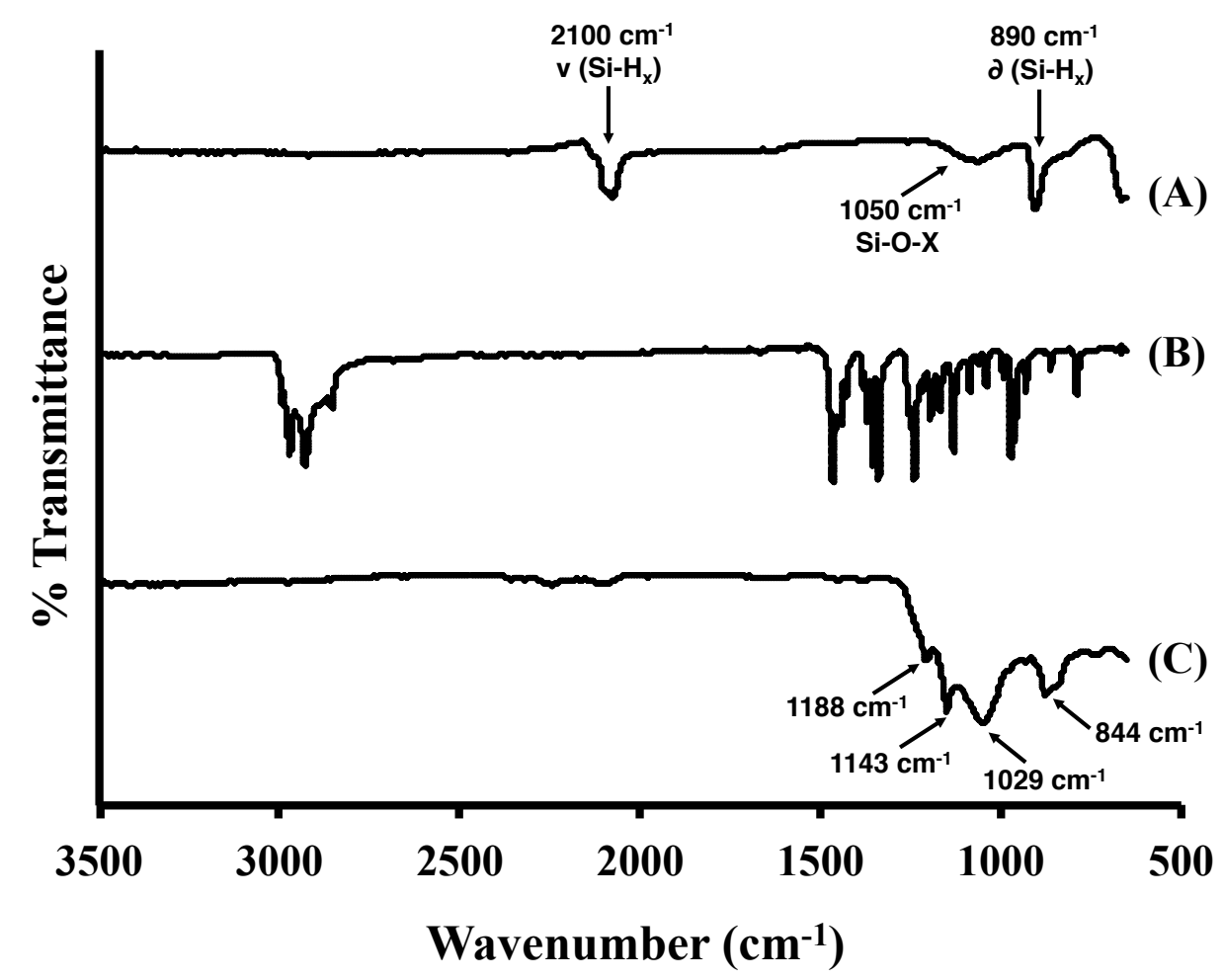

Figure 4.8. FT-IR spectra of (A) H-Si NPs, (B) TEMPO and (C) Si NPs isolated from $8.0 \mathrm{mM}$ TEMPO $48 \mathrm{~h}$ after sample preparation.

reported in the literature. For example, silicon-oxide species such as the non-bridging oxygen hole $(\mathrm{NBOH})$ center have previously has been identified as the source of $720 \mathrm{~nm}$ PL from $\mathrm{p}-\mathrm{Si}{ }^{69}$ Formation of Si-O bonding on the surfaces of the photo-excited Si NPs likely occurred as a result of reaction with trace oxygen impurities present in the suspensions, independent of TEMPO; this explanation would account for the oxidation observed by FT-IR in Chapter 3 where no TEMPO was present. However, in the presence of TEMPO and UV light, we propose that quenching of the original $660 \mathrm{~nm}$ PL occurs via an oxidative quenching mechanism, which effectively increases the rate of oxidation of the Si NP surface and the formation of the $575 \mathrm{~nm}$ PL band. 


\subsection{Preliminary conclusions}

To summarize, TEMPO was added to suspensions of red-emitting H-Si NPs to probe the stability of interband PL in the presence of a radical trap. TEMPO was observed to initially quench low energy Si NP PL, likely via an oxidative quenching mechanism. Subsequently, brightening is observed, which is likely due to Si• trapping by TEMPO radicals, which suppresses non-radiative recombination. However, at longer time points, further changes to the low energy, or interband, PL were dependent on the conditions in which the samples were aged. When the samples were stored in the dark at $4^{\circ} \mathrm{C}$, a gradual hypsochromic shift in the low energy PL was observed. We propose that this was due to interband PL from Si NPs with smaller diameters than the original Si NPs. When the samples were photo-irradiated once every ten minutes and kept at room temperature, the $660 \mathrm{~nm}$ emission was replaced by a $575 \mathrm{~nm}$ emission. We assign the $575 \mathrm{~nm}$ transition as due to an oxide-related defect state resulting from rapid oxidation of photo-excited Si NPs. 


\section{Chapter 5}

Conclusions

The studies described herein analyzed the stability of the H-Si NP surface under an array of chemical and physical conditions. First, evidence of $\mathrm{Si}-\mathrm{C}$ bonding was observed by FT-IR spectroscopy after heating in $n$-hexane under mild conditions, which showed that it was possible to elicit partial alkylation of the Si NP surface under mild conditions. This observation indicated the presence of reactive Si-based radicals on the surfaces of H-Si NPs following extraction from the chemical etch. Next, we attempted to stabilize the H-Si NP surfaces by eliminating the surface radicals implicated in the surface alkylation mechanisms. The reactivity between the H-Si NPs and TEMPO, a stable radical that has been shown to react with Si radicals, was shown to be heavily dependent on the reaction conditions.

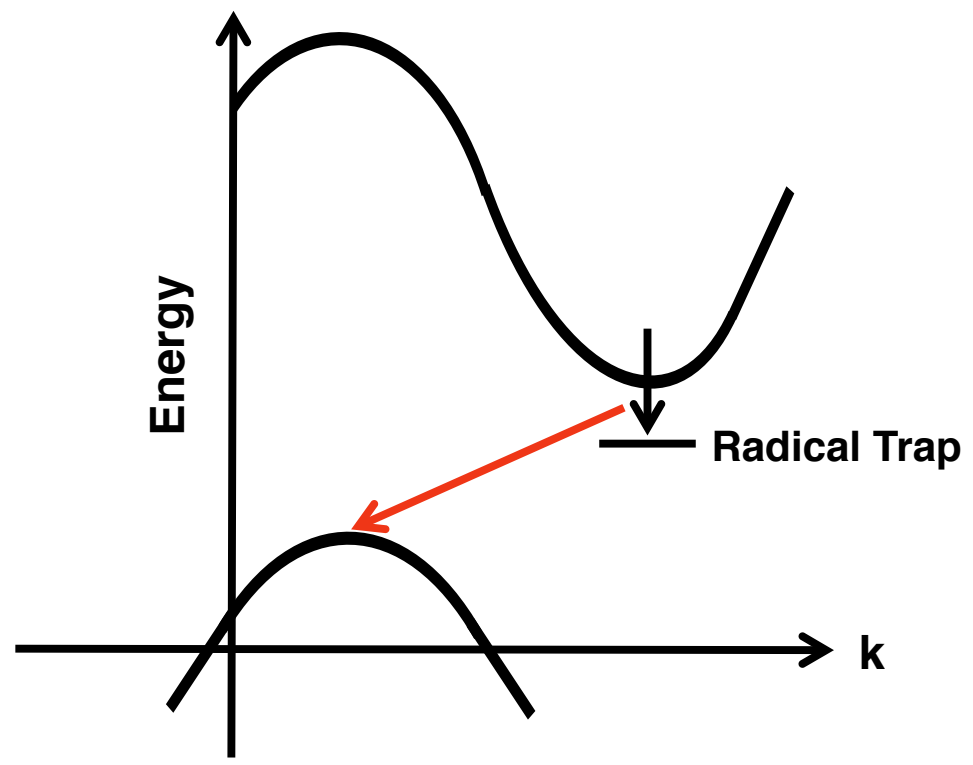

Figure 5.1. Simplified energy diagram of un-oxidized H-Si NPs showing the interband transition (red arrow) and carrier trapping by a radical trap below the conduction band minimum (black arrow) 
As a result of all data collected and analyzed, we propose the following energy diagrams to account for our observations. We propose Figure 5.1 as a simplified energy diagram of un-oxidized H-Si NPs. When strong Si-H vibrational modes were observed by FT-IR spectroscopy, the Si NP suspensions exhibited strong, red PL. We propose that the red PL is due to radiative recombination of charge carriers across the bandgap (red arrow). We furthermore propose that the radicals implicated in the surface alkylation result in the presence of trap states below the conduction band minimum, since trapping of photogenerated charge carriers at mid-gap trap states (black arrow) would result in a reduction in the radiative yield of interband PL.

In general, changes in the surface composition of the initially hydride-terminated Si NPs were accompanied by changes in the optical properties of the suspensions. Loss of Si-H bonding following the heating of H-Si NPs in refluxing $n$-hexane was concurrent with loss of the red, interband PL typical of colloidal H-Si NPs. A reduction in the Si-H bonding features was also observed following light-mediated reaction of H-Si NPs with TEMPO, which was also concurrent with loss of interband PL. In both studies, loss of Si$\mathrm{H}$ bonding features was also accompanied by an increase in the intensity of Si-O bonding features.

Differences in the conditions of oxide formation resulted in differences in the observed PL. Figure 5.2 is a simplified energy diagram showing how modification of the Si NP core with a surface oxide may affect the electronic structure of the system. In Chapter 3 and the first study of Chapter 4, when the surface oxide was formed at slightly elevated temperatures (greater than room temperature), the resulting Si NP suspensions exhibited intense blue PL. However, when the surface oxide was formed under UV- 
irradiation, the resulting Si NP suspensions exhibited intense orange PL. The vibrational modes present in the Si-O region of the IR spectra were likewise dependent on the conditions in which the surface oxide was formed. However, we propose that both the orange and blue PL result from radiative defect states located at the interface between the Si NP core and the surface oxide (blue and orange arrows). The data presented herein support our hypothesis that H-Si NPs are highly unstable, and prone to oxidation. As such, optical applications for Si NPs remain heavily limited, until methods for prevention or controlled oxidation of the Si NP surface are developed.

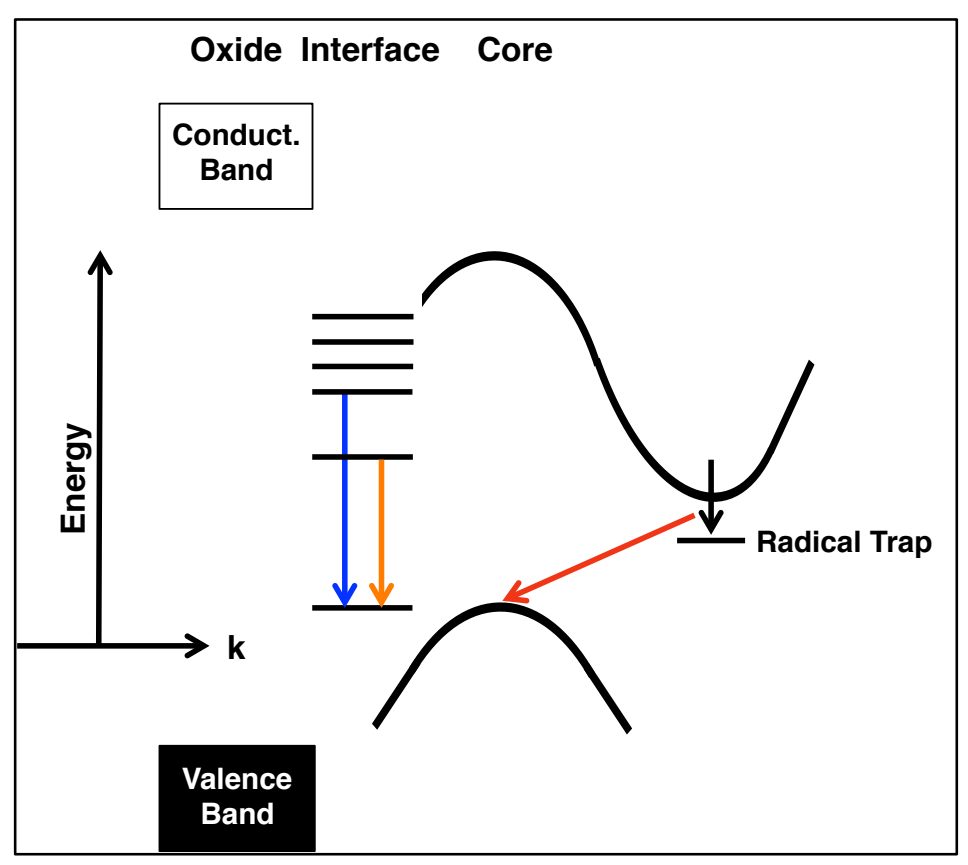

Figure 5.2. Simplified energy diagram of oxidized Si NPs showing the interband transition (red arrow) and carrier trapping by a radical trap below the conduction band minimum (black arrow) of the Si NP core. The electronically inactive conduction and valence band extrema of silicon oxide are shown, and lie outside of the Si NP bandgap. Decay from defect states at the interface of the Si NP core and the surface oxide are shown (blue and orange arrows). 
(1) Sanchez, C.; Belleville, P.; Popall, M.; Nicole, L. Applications of Advanced Hybrid Organic-inorganic Nanomaterials: From Laboratory to Market. Chem. Soc. Rev. 2011, 40, 696-753.

(2) Rao, C. N. R.; Govindaraj, A.; Vivekchand, S. R. C. Inorganic Nanomaterials: Current Status and Future Prospects. Annu. Rep. Prog. Chem., Sect. A Inorg. Chem. 2006, 102, 20-45.

(3) Daniel, M.-C.; Astruc, D. Gold Nanoparticles: Assembly, Supramolecular Chemistry, Quantum-Size-Related Properties, and Applications toward Biology, Catalysis, and Nanotechnology. Chem. Rev. 2004, 104, 293-346.

(4) Algar, W. R.; Tavares, A. J.; Krull, U. J. Beyond Labels: A Review of the Application of Quantum Dots as Integrated Components of Assays, Bioprobes, and Biosensors Utilizing Optical Transduction. Anal. Chim. Acta 2010, 673, 1-25.

(5) Singh, J. Semiconductor Devices : Basic Principles; Wiley, 2000.

(6) Trwoga, P. F.; Kenyon, A. J.; Pitt, C. W. Modeling the Contribution of Quantum Confinement to Luminescence from Silicon Nanoclusters. J. Appl. Phys. 1998, 83, 3789-3794.

(7) Bawendi, M. G.; Steigerwald, M. L.; Brus, L. E. The Quantum Mechanics of Larger Semiconductor Clusters ("Quantum Dots"). Ann. Rev. Phys. Chem. 1990, 41, 477-496.

(8) Brus, L. E. Luminescence of Silicon Materials: Chains, Sheets, Nanocrystals, Nanowires, Microcrystals, and Porous Silicon. J. Phys. Chem. 1994, 98, 35753581 .

(9) Hessel, C. M.; Reid, D.; Panthani, M. G.; Rasch, M. R.; Goodfellow, B. W.; Wei, J.; Fujii, H.; Akhavan, V.; Korgel, B. A. Synthesis of Ligand-Stabilized Silicon Nanocrystals with Size-Dependent Photoluminescence Spanning Visible to NearInfrared Wavelengths. Chem. Mater. 2012, 24, 393-401.

(10) Prokes, S. M. Surface and Optical Properties of Porous Silicon. J. Mater. Res. 1995, 11, 305-320.

(11) Alivisatos, A. P. Perspectives on the Physical Chemistry of Semiconductor Nanocrystals. J. Phys. Chem. 1996, 100, 13226-13239.

(12) Nayfeh, M. H.; Rao, S.; Barry, N.; Therrien, J.; Belomoin, G.; Smith, A.; Chaieb, S. Observation of Laser Oscillation in Aggregates of Ultrasmall Silicon Nanoparticles. Appl. Phys. Lett. 2002, 80, 121-123.

(13) Yang, S.; Li, W.; Cao, B.; Zeng, H.; Cai, W. Origin of Blue Emission from Silicon Nanoparticles: Direct Transition and Interface Recombination. J. Phys. Chem. C 2011, 115, 21056-21062.

(14) Bardeen, J. Semiconductor Research Leading to the Point Contact Transistor. Nobel Lect. Phys. 1942-1962 1956, 318-341.

(15) Uhlir Jr., A. Electrolytic Shaping of Germanium and Silicon. Bell Syst. Tech. J. 1956, 35, 333-347.

(16) Uhlir, A.; Uhlir, I. (Williams). Historical Perspective on the Discovery of Porous Silicon. Phys. Status Solidi 2005, 2, 3185-3187. 
(17) Turner, D. R. Electropolishing Silicon in Hydrofluoric Acid Solutions. $J$. Electrochem. Soc. 1958, 105, 402-408.

(18) Chuang, S.-F.; Collins, S. D.; Smith, R. L. Porous Silicon Microstructure as Studied by Transmission Electron Microscopy. Appl. Phys. Lett. 1989, 55, 15401542.

(19) Canham, L. T. Silicon Quantum Wire Array Fabrication by Electrochemical and Chemical Dissolution of Wafers. Appl. Phys. Lett. 1990, 57, 1046-1048.

(20) Liu, S.; Sato, S.; Kimura, K. Synthesis of Luminescent Silicon Nanopowders Redispersible to Various Solvents. Langmuir 2005, 21, 6324-6329.

(21) Hessel, C. M.; Henderson, E. J.; Veinot, J. G. C. Hydrogen Silsesquioxane : A Molecular Precursor for Nanocrystalline Si-SiO2 Composites and Freestanding Hydride-Surface-Terminated Silicon Nanoparticles. Chem. Mater. 2006, 18, 61396146.

(22) Manhat, B. A.; Brown, A. L.; Black, L. A.; Ross, J. B. A.; Fichter, K.; Vu, T.; Richman, E.; Goforth, A. M. One-Step Melt Synthesis of Water Soluble, Photoluminescent, Surface-Oxidized Silicon Nanoparticles for Cellular Imaging Applications. Chem. Mater. 2011, 23, 2407-2418.

(23) Chiu, S.-K.; Manhat, B. A.; DeBenedetti, W. J. I.; Brown, A. L.; Fichter, K.; Vu, T.; Eastman, M.; Jiao, J.; Goforth, A. M. Aqueous Red-Emitting Silicon Nanoparticles for Cellular Imaging: Consequences of Protecting against Surface Passivation by Hydroxide and Water for Stable Red Emission. J. Mater. Res. 2013, 28, 216-230.

(24) Bley, R. A.; Kauzlarich, S. M. A Low-Temperature Solution Phase Route for the Synthesis of Silicon Nanoclusters. J. Am. Chem. Soc. 1996, 118, 12461-12462.

(25) Dhas, N. A.; Raj, C. P.; Gedanken, A. Preparation of Luminescent Silicon Nanoparticles: A Novel Sonochemical Approach. Chem. Mater. 1998, 10, 32783281.

(26) Baldwin, R. K.; Pettigrew, K. A.; Ratai, E.; Augustine, M. P.; Kauzlarich, S. M. Solution Reduction Synthesis of Surface Stabilized Silicon Nanoparticles. Chem. Comm. 2002, 1822-1823.

(27) Baldwin, R. K.; Pettigrew, K. a; Garno, J. C.; Power, P. P.; Liu, G.; Kauzlarich, S. M. Room Temperature Solution Synthesis of Alkyl-Capped Tetrahedral Shaped Silicon Nanocrystals. J. Am. Chem. Soc. 2002, 124, 1150-1151.

(28) Zhang, X.; Neiner, D.; Wang, S.; Louie, A. Y.; Kauzlarich, S. M. A New Solution Route to Hydrogen-Terminated Silicon Nanoparticles: Synthesis, Functionalization and Water Stability. Nanotechnology 2007, 18, 095601-095607.

(29) Balc1, M. H.; Maria, J.; Vullum-Bruer, F.; Lindgren, M.; Grande, T.; Einarsrud, M.-A. Synthesis of Monodisperse Silicon Quantum Dots Through a K-Naphthalide Reduction Route. J. Clust. Sci. 2012, 23, 421-435.

(30) Li, X.; He, Y.; Talukdar, S. S.; Swihart, M. T. Process for Preparing Macroscopic Quantities of Brightly Photoluminescent Silicon Nanoparticles with Emission Spanning the Visible Spectrum. Langmuir 2003, 19, 8490-8496.

(31) Sankaran, R. M.; Holunga, D.; Flagan, R. C.; Giapis, K. P. Synthesis of Blue Luminescent Si Nanoparticles Using Atmospheric-Pressure Microdischarges. Nano Lett. 2005, 5, 537-541. 
(32) Mangolini, L.; Jurbergs, D.; Rogojina, E.; Kortshagen, U. High Efficiency Photoluminescence from Silicon Nanocrystals Prepared by Plasma Synthesis and Organic Surface Passivation. Phys. Stat. Sol. 2006, 3, 3975-3978.

(33) Vorotyntsev, A. V.; Mochalov, G. M.; Vorotyntsev, V. M. Kinetics of Catalytic Hydrogen Reduction of SiCl4 in the Presence of Nickel Chloride. Inorg. Mater. 2012, 49, 1-5.

(34) Cheng, X.; Lowe, S. B.; Reece, P. J.; Gooding, J. J. Colloidal Silicon Quantum Dots: From Preparation to the Modification of Self-Assembled Monolayers (SAMs) for Bio-Applications. Chem. Soc. Rev. 2014, 43, 2680-2700.

(35) Pavesi, L.; Turan, R. Silicon Nanocrystals : Fundamentals, Synthesis and Applications; Wiley-VCH, 2010.

(36) Content, S.; Trogler, W. C.; Sailor, M. J. Detection of Nitrobenzene, DNT , and TNT Vapors by Quenching of Porous Silicon Photoluminescence. Chem. Eur. J. 2000, 6, 2205-2213.

(37) Han, Y.; Chen, Y.; Feng, J.; Liu, J.; Ma, S.; Chen, X. One-Pot Synthesis of Fluorescent Silicon Nanoparticles for Sensitive and Selective Determination of 2,4,6-Trinitrophenol in Aqueous Solution. Anal. Chem. 2017, 89, 3001-3008.

(38) Wang, H.; He, Y. Recent Advances in Silicon Nanomaterial-Based Fluorescent Sensors. Sensors 2017, 17, 268.

(39) Xu, X.; Ma, S.; Xiao, X.; Hu, Y.; Zhao, D.; Yang, L.; Su, Y.; Lee, S.; He, Y.; Chen, Z.; Prasad, P. N.; Yang, B. The Preparation of High-Quality Water-Soluble Silicon Quantum Dots and Their Application in the Detection of Formaldehyde. RSC Adv. 2016, 6, 98899-98907.

(40) Rogozhina, E. V.; Eckhoff, D. A.; Gratton, E.; Braun, P. V. Carboxyl Functionalization of Ultrasmall Luminescent Silicon Nanoparticles through Thermal Hydrosilylation. J. Mater. Chem. 2006, 16, 1421-1430.

(41) Erogbogbo, F.; Tien, C.-A.; Chang, C.-W.; Yong, K.-T.; Law, W.-C.; Ding, H.; Roy, I.; Swihart, M. T.; Prasad, P. N. Bioconjugation of Luminescent Silicon Quantum Dots for Selective Uptake by Cancer Cells. Bioconjugate Chem. 2011, 22, 1081-1088.

(42) Zhong, Y.; Peng, F.; Bao, F.; Wang, S.; Ji, X.; Yang, L.; Su, Y.; Lee, S.-T.; He, Y. Large-Scale Aqueous Synthesis of Fluorescent and Biocompatible Silicon Nanoparticles and Their Use as Highly Photostable Biological Probes. J. Am. Chem. Soc. 2013, 135, 8350-8356.

(43) Jung, K. H.; Shih, S.; Hsieh, T. Y.; Kwong, D. L.; Lin, T. L. Intense Photoluminescence from Laterally Anodized Porous Si. Appl. Phys. Lett. 1991, 59, 3264-3266.

(44) Maier-Flaig, F.; Rinck, J.; Stephan, M.; Bocksrocker, T.; Bruns, M.; Kübel, C.; Powell, A. K.; Ozin, G. A.; Lemmer, U. Multicolor Silicon Light-Emitting Diodes (SiLEDs). Nano Lett. 2013, 13, 475-480.

(45) Li, Z. F.; Ruckenstein, E. Water-Soluble Poly(acrylic Acid) Grafted Luminescent Silicon Nanoparticles and Their Use as Fluorescent Biological Staining Labels. Nano Lett. 2004, 4, 1463-1467. 
(46) Canham, L. T.; Loni, A.; Calcott, P. D. J.; Simons, A. J.; Reeves, C.; Houlton, M. R.; Newey, J. P.; Nash, K. J.; Cox, T. I. On the Origin of Blue Luminescence Arising from Atmospheric Impregnation of Oxidized Porous Silicon. Thin Solid Films 1996, 276, 112-115.

(47) Valizadeh, A.; Mussa Farkhani, S. Review: Three Synthesis Methods of CdX (X = $\mathrm{Se}, \mathrm{S}$ or Te) Quantum Dots. IET Nanobiotechnology 2014, 8, 59-76.

(48) Zolotarev, K. V.; Kashirtseva, V. N.; Mishin, A. V.; Belyaeva, N. F.; Medvedeva, N. V.; Ipatova, O. M. Assessment of Toxicity of Cdse/Cds/Zns/S,S-Dihydrolipoic Acid/Polyacrylic Acid Quantum Dots at Danio Rerio Embryos and Larvae. ISRN Nanotechnol. 2012, 1-5.

(49) Delerue, C.; Allan, G.; Lannoo, M. Theoretical Aspects of the Luminescence of Porous Silicon. Phys. Rev. B 1993, 48, 24-36.

(50) Schmitt-Rink, S.; Miller, D. A. B.; Chemla, D. S. Theory of the Linear and Nonlinear Optical Properties of Semiconductor Microcrystallites. Phys. Rev. B 1987, 35, 8113-8125.

(51) Dasog, M.; De los Reyes, G. B.; Titova, L. V.; Hegmann, F. A.; Veinot, J. G. C. Size vs Surface : Tuning the Photoluminescence of Freestanding Silicon Nanocrystals Across the Visible Spectrum via Surface Groups. ACS Nano 2014, 8, 9636-9648.

(52) Wolkin, M. V.; Jorne, J.; Fauchet, P. M.; Allan, G.; Delerue, C. Electronic States and Luminescence in Porous Silicon Quantum Dots: The Role of Oxygen. Phys. Rev. Lett. 1999, 82, 197-200.

(53) Martínez, A.; Alonso, J. C.; Sansores, L. E.; Salcedo, R. Electronic Structure of Silicon Nanocrystals Passivated with Nitrogen and Chlorine. J. Phys. Chem. C 2010, 114, 12427-12431.

(54) Puzder, A.; Williamson, A. J.; Grossman, J. C.; Galli, G. Surface Chemistry of Silicon Nanoclusters. Phys. Rev. Lett. 2002, 88, 97401.

(55) Dohnalová, K.; Gregorkiewicz, T.; Kůsová, K. Silicon Quantum Dots: Surface Matters. J. Phys. Condens. Matter 2014, 26, 173201.

(56) Gupta, A.; Swihart, M. T.; Wiggers, H. Luminescent Colloidal Dispersion of Silicon Quantum Dots from Microwave Plasma Synthesis: Exploring the Photoluminescence Behavior Across the Visible Spectrum. Adv. Funct. Mater. 2009, 19, 696-703.

(57) Hiller, D.; Goetze, S.; Munnik, F.; Jivanescu, M.; Gerlach, J. W.; Vogt, J.; Pippel, E.; Zakharov, N.; Stesmans, A.; Zacharias, M. Nitrogen at the Si-nanocrystal/SiO2 Interface and Its Influence on Luminescence and Interface Defects. Phys. Rev. B 2010, 82, 195401.

(58) Kanemitsu, Y. Luminescence Properties of Nanometer-Sized Si Crystallites: Core and Surface States. Phys. Rev. B 1994, 49, 845-848.

(59) Ma, Y.; Chen, X.; Pi, X.; Yang, D. Theoretical Study of Chlorine for Silicon Nanocrystals. J. Phys. Chem. C 2011, 115, 12822-12825.

(60) Warner, J. H.; Rubinsztein-Dunlop, H.; Tilley, R. D. Surface Morphology Dependent Photoluminescence from Colloidal Silicon Nanocrystals. J. Phys. Chem. B 2005, 109, 19064-19067. 
(61) Zhou, Z.; Brus, L. E.; Friesner, R. Electronic Structure and Luminescence of 1.1and 1.4-Nm Silicon Nanocrystals: Oxide Shell versus Hydrogen Passivation. Nano Lett. 2003, 3, 163-167.

(62) Clark, R. J.; Dang, M. K. M.; Veinot, J. G. C. Exploration of Organic Acid Chain Length on Water-Soluble Silicon Quantum Dot Surfaces. Langmuir 2010, 26, 15657-15664.

(63) Dasog, M.; Yang, Z.; Regli, S.; Atkins, T. M.; Faramus, A.; Singh, M. P.; Muthuswamy, E.; Kauzlarich, S. M.; Tilley, R. D.; Veinot, J. G. C. Chemical Insight into the Origin of Red and Blue Photoluminescence Arising from Freestanding Silicon Nanocrystals. ACS Nano 2013, 7, 2676-2685.

(64) Heintz, A. S.; Fink, M. J.; Mitchell, B. S. Silicon Nanoparticles with Chemically Tailored Surfaces. Appl. Organometal. Chem. 2010, 24, 236-240.

(65) Kanemitsu, Y.; Ogawa, T.; Shiraishi, K.; Takeda, K. Visible Photoluminescence from Oxidized Si Nanometer-Sized Spheres: Exciton Confinement on a Spherical Shell. Phys. Rev. B 1993, 48, 4883-4887.

(66) Kanemitsu, Y.; Futagi, T.; Matsumoto, T.; Mimura, H. Origin of the Blue and Red Photoluminescence from Oxidized Porous Silicon. Phys. Rev. B 1994, 49, 732736.

(67) Debenedetti, W. J. I.; Chiu, S. K.; Radlinger, C. M.; Ellison, R. J.; Manhat, B. A.; Zhang, J. Z.; Shi, J.; Goforth, A. M. Conversion from Red to Blue Photoluminescence in Alcohol Dispersions of Alkyl-Capped Silicon Nanoparticles: Insight into the Origins of Visible Photoluminescence in Colloidal Nanocrystalline Silicon. J. Phys. Chem. C 2015, 119, 9595-9608.

(68) Munekuni, S.; Yamanaka, T.; Shimogaichi, Y.; Tohmon, R.; Ohki, Y.; Nagasawa, K.; Hama, Y. Various Types of Nonbridging Oxygen Hole Center in High-Purity Silica Glass. J. Appl. Phys. 1990, 68, 1212-1217.

(69) Prokes, S. M.; Glembocki, O. J. Role of Interfacial Oxide-Related Defects in the Red-Light Emission in Porous Silicon. Phys. Rev. B 1993, 49, 2238-2241.

(70) Kanemitsu, Y. Slow Decay Dynamics of Visible Luminescence in Porous Silicon: Hopping of Carriers Confined on a Shell Region in Nanometer-Size Si Crystallites. Phys. Rev. B 1993, 48, 357-360.

(71) Tsu, R. Phenomena in Silicon Nanostructure Devices. Appl. Phys. A 2000, 71, 391-402.

(72) López, M.; Garrido, B.; García, C.; Pellegrino, P.; Pérez-Rodríguez, A.; Morante, J. R.; Bonafos, C.; Carrada, M.; Claverie, A. Elucidation of the Surface Passivation Role on the Photoluminescence Emission Yield of Silicon Nanocrystals Embedded in SiO2. Appl. Phys. Lett. 2002, 80, 1637-1639.

(73) Niesar, S.; Stegner, A. R.; Pereira, R. N.; Hoeb, M.; Wiggers, H.; Brandt, M. S.; Stutzmann, M. Defect Reduction in Silicon Nanoparticles by Low-Temperature Vacuum Annealing. Appl. Phys. Lett. 2010, 96, 193112.

(74) Hahn, T.; Heimfarth, J. P.; Roewer, G.; Kroke, E. Silicon Nano Particles: Surface Characterization, Defects and Electronic Properties. Phys. Stat. Sol. 2008, 245, 959-962. 
(75) Vella, E.; Messina, F.; Cannas, M.; Boscaino, R. Unraveling Exciton Dynamics in Amorphous Silicon Dioxide: Interpretation of the Optical Features from 8 to 11 eV. Phys. Rev. B 2011, 83, 174201.

(76) Buriak, J. M.; Stewart, M. P.; Geders, T. W.; Allen, M. J.; Choi, H. C.; Smith, J.; Raftery, D.; Canham, L. T. Lewis Acid Mediated Hydrosilylation on Porous Silicon Surfaces. J. Am. Chem. Soc. 1999, 121, 11491-11502.

(77) Linford, M. R.; Chidsey, C. E. D. Alkyl Monolayers Covalently Bonded to Silicon Surfaces. J. Am. Chem. Soc. 1993, 115, 12631-12632.

(78) Buriak, J. M. Organometallic Chemistry on Silicon and Germanium Surfaces. Chem. Rev. 2002, 102, 1271-1308.

(79) Buriak, J. M.; Allen, M. J. Lewis Acid Mediated Functionalization of Porous Silicon with Substituted Alkenes and Alkynes. J. Am. Chem. Soc. 1998, 120, $1339-1340$.

(80) Stewart, M. P.; Robins, E. G.; Geders, T. W.; Allen, M. J.; Choi, H. C.; Buriak, J. M. Three Methods for Stabilization and Functionalization of Porous Ssilicon Ssurfaces via Hydrosilylation and Electrografting Rreactions. Phys. Stat. Sol. 2000, 182, 109-115.

(81) Linford, M. R.; Fenter, P.; Eisenberger, P. M.; Chidsey, C. E. D. Alkyl Monolayers on Silicon Prepared from 1-Alkenes and Hydrogen-Terminated Silicon. J. Am. Chem. Soc. 1995, 117, 3145-3155.

(82) Wheeler, L. M.; Anderson, N. C.; Palomaki, P. K. B.; Blackburn, J. L.; Johnson, J. C.; Neale, N. R. Silyl Radical Abstraction in the Functionalization of PlasmaSynthesized Silicon Nanocrystals. Chem. Mater. 2015, 27, 6869-6878.

(83) Boukherroub, R.; Morin, S.; Wayner, D. D. M.; Bensebaa, F.; Sproule, G. I.; Baribeau, J.-M.; Lockwood, D. J. Ideal Passivation of Luminescent Porous Silicon by Thermal, Noncatalytic Reaction with Alkenes and Aldehydes. Chem. Mater. 2001, 13, 2002-2011.

(84) Sieval, A. B.; Demirel, A. L.; Nissink, J. W. M.; Linford, M. R.; van der Maas, J. H.; de Jeu, W. H.; Zuilhof, H.; Sudho, E. J. R. Highly Stable Si - C Linked Functionalized Monolayers on the Silicon ( 100 ) Surface. Langmuir 1998, 14, $1759-1768$.

(85) Bateman, J. E.; Eagling, R. D.; Worrall, D. R.; Horrocks, B. R.; Houlton, A. Alkylation of Porous Silicon by Direct Reaction with Alkenes and Alkynes. Angew. Chem. Int. Ed. 1998, 37, 2683-2685.

(86) Boukherroub, R.; Morin, S.; Wayner, D. D. M.; Lockwood, D. J. Thermal Route for Chemical Modification and Photoluminescence Stabilization of Porous Silicon. Phys. Stat. Sol. 2000, 182, 117-121.

(87) Cicero, R. L.; Linford, M. R.; Chidsey, C. E. D. Photoreactivity of Unsaturated Compounds with Hydrogen-Terminated Silicon(111). Langmuir 2000, 16, 56885695.

(88) Stewart, M. P.; Buriak, J. M. Exciton-Mediated Hydrosilylation on Photoluminescent Nanocrystalline Silicon. J. Am. Chem. Soc. 2001, 123, 78217830 . 
(89) Tian, R.; Seitz, O.; Li, M.; Hu, W. (Walter); Chabal, Y. J.; Gao, J. Infrared Characterization of Interfacial Si-O Bond Formation on Silanized Flat $\mathrm{SiO} 2 / \mathrm{Si}$ Surfaces. Langmuir 2010, 26, 4563-4566.

(90) Bateman, J. E.; Eagling, R. D.; Horrocks, B. R.; Houlton, A.; Worrall, D. R. Role for Organic Molecules in the Oxidation of Porous Silicon. Chem Commun. 1997, 2275-2276.

(91) Liu, S.-M.; Yang, Y.; Sato, S.; Kimura, K. Enhanced Photoluminescence from Si Nano-Organosols by Functionalization with Alkenes and Their Size Evolution. Chem. Mater. 2006, 18, 637-642.

(92) Rosso-Vasic, M.; Spruijt, E.; van Lagen, B.; De Cola, L.; Zuilhof, H. AlkylFunctionalized Oxide-Free Silicon Nanoparticles: Synthesis and Optical Properties. Small 2008, 4, 1835-1841.

(93) Pettigrew, K. A.; Liu, Q.; Power, P. P.; Kauzlarich, S. M. Solution Synthesis of Alkyl- and Alkyl / Alkoxy-Capped Silicon Nanoparticles via Oxidation of Mg2Si. Chem. Mater. 2003, 15, 4005-4011.

(94) Vincent, J.; Maurice, V.; Paquez, X.; Sublemontier, O.; Leconte, Y.; Guillois, O.; Reynaud, C.; Herlin-Boime, N.; Raccurt, O.; Tardif, F. Effect of Water and UV Passivation on the Luminescence of Suspensions of Silicon Quantum Dots. $J$. Nanopart. Res. 2010, 12, 39-46.

(95) Holmes, J. D.; Ziegler, K. J.; Doty, R. C.; Pell, L. E.; Johnston, K. P.; Korgel, B. A. Highly Luminescent Silicon Nanocrystals with Discrete Optical Transitions. $J$. Am. Chem. Soc. 2001, 123, 3743-3748.

(96) Himcinschi, C.; Milekhin, A.; Friedrich, M.; Hiller, K.; Wiemer, M.; Gessner, T.; Schulze, S.; Zahn, D. R. T. Silicon Oxide in Si-Si Bonded Wafers. Appl. Surf. Sci. 2001, 175-176, 715-720.

(97) Li, S.; Silvers, S. J.; El-Shall, M. S. Surface Oxidation and Luminescence Properties of Weblike Agglomeration of Silicon Nanocrystals Produced by a Laser Vaporization-Controlled Condensation Technique. J. Phys. Chem. B 1997, 101, 1794-1802.

(98) X-ray Photoelectron Spectroscopy (XPS) Reference Pages: What is Adventitious Carbon? http://www.xpsfitting.com/2011/01/what-is-adventitious-carbon.html (accessed Apr 5, 2017).

(99) Panthani, M. G.; Hessel, C. M.; Reid, D.; Casillas, G.; Jose, M.; Korgel, B. A. Graphene-Supported High-Resolution TEM and STEM Imaging of Silicon Nanocrystals and Their Capping Ligands. J. Phys. Chem. C 2012, 116, 2246322468.

(100) Hartstein, A.; Tsang, J. C.; Dimaria, D. J.; Dong, D. W. Observation of Amorphous Silicon Regions in Silicon-Rich Silicon Dioxide Films. Appl. Phys. Lett. 1980, 36, 836-837.

(101) Mamiya, M.; Kikuchi, M.; Takei, H. Crystallization of Fine Silicon Particles from Silicon Monoxide. J. Cryst. Growth 2002, 237-239, 1909-1914.

(102) Ke, W.; Feng, X.; Huang, Y. Annealing Effects on the Size of Si-Nanocrystals Embedded in Bulk SiO. J. Cryst. Growth 2011, 316, 191-195. 
(103) Mamiya, M.; Takei, H.; Kikuchi, M.; Uyeda, C. Preparation of Fine Silicon Particles from Amorphous Silicon Monoxide by the Disproportionation Reaction. J. Cryst. Growth 2001, 229, 457-461.

(104) Sun, W.; Qian, C.; Cui, X. S.; Wang, L.; Wei, M.; Casillas, G.; Helmy, A. S.; Ozin, G. A. Silicon Monoxide - a Convenient Precursor for Large Scale Synthesis of near Infrared Emitting Monodisperse Silicon Nanocrystals. Nanoscale 2016, 8, 3678-3684.

(105) Zhang, R. Q.; Zhao, M. W.; Lee, S. T. Silicon Monoxide Clusters: The Favorable Precursors for Forming Silicon Nanostructures. Phys. Rev. Lett. 2004, 93, 7-10.

(106) Sakko, A.; Sternemann, C.; Sahle, C. J.; Sternemann, H.; Feroughi, O. M.; Conrad, H.; Djurabekova, F.; Hohl, A.; Seidler, G. T.; Tolan, M.; Hämäläinen, K. Suboxide Interface in Disproportionating a -SiO Studied by X-Ray Raman Scattering. Phys. Rev. B 2010, 81, 205317.

(107) Kapaklis, V.; Politis, C.; Poulopoulos, P.; Schweiss, P. Photoluminescence from Silicon Nanoparticles Prepared from Bulk Amorphous Silicon Monoxide by the Disproportionation Reaction. Appl. Phys. Lett. 2005, 87, 123114.

(108) Dasog, M.; Veinot, J. G. C. Size Independent Blue Luminescence in Nitrogen Passivated Silicon Nanocrystals. Phys. Stat. Sol. 2012, 209, 1844-1846.

(109) Trucks, G. W.; Raghavachari, K.; Hicashi, G. S.; Chabal, Y. J. Mechanism of HF Etching of Silicon Surfaces: A Theoretical Understanding of Hydrogen Passivation. Phys. Rev. Lett. 1990, 65, 504-507.

(110) Yang, Z.; Iqbal, M.; Dobbie, A. R.; Veinot, J. G. C. Surface-Induced Alkene Oligomerization: Does Thermal Hydrosilylation Really Lead to Monolayer Protected Silicon Nanocrystals? J. Am. Chem. Soc. 2013, 135, 17595-17601.

(111) Woods, M.; Carlsson, S.; Hong, Q.; Patole, S. N.; Lie, L. H.; Houlton, A.; Horrocks, B. R. A Kinetic Model of the Formation of Organic Monolayers on Hydrogen-Terminated Silicon by Hydrosilation of Alkenes. J. Phys. Chem. B 2005, 109, 24035-24045.

(112) Bech Nielsen, B.; Hoffmann, L.; Budde, M. Si-H Stretch Modes of Hydrogen Vacancy Defects in Silicon. Mater. Sci. Eng. B 1996, 36, 259-263.

(113) Kim, N. Y.; Laibinis, P. E. Thermal Derivatization of Porous Silicon with Alcohols. J. Am. Chem. Soc. 1997, 119, 2297-2298.

(114) Klanjsek Gunde, M. Vibrational Modes in Amorphous Silicon Dioxide. Phys. $B$ 2000, 286-295.

(115) Li, P.; Wang, G.; Ma, Y.; Fang, R. Origin of the Blue and Red Photoluminescence from Aged Porous Silicon. Phys. Rev. B 1998, 58, 4057-4065.

(116) Jurbergs, D.; Rogojina, E.; Mangolini, L.; Kortshagen, U. Silicon Nanocrystals with Ensemble Quantum Yields Exceeding 60\%. Appl. Phys. Lett. 2006, 88, 233116.

(117) Heintz, A. S.; Fink, M. J.; Mitchell, B. S. Mechanochemical Synthesis of Blue Luminescent Alkyl/Alkenyl-Passivated Silicon Nanoparticles. Adv. Mater. 2007, 19, 3984-3988.

(118) Veinot, J. G. C. Synthesis, Surface Functionalization, and Properties of Freestanding Silicon Nanocrystals. Chem. Commun. 2006, 4160-4168. 
(119) Michalak, D. J.; Amy, S. R.; Aureau, D.; Dai, M.; Estève, A.; Chabal, Y. J. Nanopatterning Si(111) Surfaces as a Selective Surface-Chemistry Route. Nat. Mater. 2010, 9, 266-271.

(120) Ling, L.; Kuwabara, S.; Abe, T.; Shimura, F. Multiple Internal Reflection Infrared Spectroscopy of Silicon Surface Structure and Oxidation Process at Room Temperature. J. Appl. Phys. 1993, 73, 3018-3022.

(121) English, D. S.; Pell, L. E.; Yu, Z.; Barbara, P. F.; Korgel, B. A. Size Tunable Visible Luminescence from Individual Organic Monolayer Stabilized Silicon Nanocrystal Quantum Dots. Nano Lett. 2002, 2, 681-685.

(122) Fuzell, J.; Thibert, A.; Atkins, T. M.; Dasog, M.; Busby, E.; Veinot, J. G. C.; Kauzlarich, S. M.; Larsen, D. S. Red States versus Blue States in Colloidal Silicon Nanocrystals: Exciton Sequestration into Low-Density Traps. J. Phys. Chem. Lett. 2013, 4, 3806-3812.

(123) Puzder, A.; Williamson, A.; Reboredo, F.; Galli, G. Structural Stability and Optical Properties of Nanomaterials with Reconstructed Surfaces. Phys. Rev. Lett. 2003, 91, 157405.

(124) Puzder, A.; Williamson, A. J.; Grossman, J. C.; Galli, G. Computational Studies of the Optical Emission of Silicon Nanocrystals. J. Am. Chem. Soc. 2003, 125, 27862791.

(125) Seraphin, A. A.; Werwa, E.; Kolenbrander, K. D. Influence of Nanostructure Size on the Luminescence Behavior of Silicon Nanoparticle Thin Films. J. Mater. Res. 2011, 12, 3386-3392.

(126) Dohnalová, K.; Kůsová, K.; Pelant, I. Time-Resolved Photoluminescence Spectroscopy of the Initial Oxidation Stage of Small Silicon Nanocrystals. Appl. Phys. Lett. 2009, 94, 211903.

(127) Sweryda-Krawiec, B.; Cassagneau, T.; Fendler, J. H. Surface Modification of Silicon Nanocrystallites by Alcohols. J. Phys. Chem. B 1999, 103, 9524-9529.

(128) Tischler, M. A.; Collins, R. T.; Stathis, J. H.; Tsang, J. C. Luminescence Degradation in Porous Silicon. Appl. Phys. Lett. 1992, 60, 639-641.

(129) Pitters, J. L.; Piva, P. G.; Tong, X.; Wolkow, R. A. Reversible Passivation of Silicon Dangling Bonds with the Stable Radical TEMPO. Nano Lett. 2003, 3, 1431-1435.

(130) Greene, M. E.; Guisinger, N. P.; Basu, R.; Baluch, A. S.; Hersam, M. C. Nitroxyl Free Radical Binding to Si(100): A Combined Scanning Tunneling Microscopy and Computational Modeling Study. Surf. Sci. 2004, 559, 16-28.

(131) Pitters, J. L.; Wolkow, R. A. Protection-Deprotection Chemistry to Control Styrene Self-Directed Line Growth on Hydrogen-Terminated Si(100). J. Am. Chem. Soc. Comm. 2005, 127, 48-49.

(132) Lopinski, G. P.; Wayner, D. D. M.; Wolkow, R. A. Self-Directed Growth of Molecular Nanostructures on Silicon. Nature 2000, 406, 48-51.

(133) Bennett, J. M.; Warschkow, O.; Marks, N. A. Aminoxyl Radicals on the Silicon (001) Surface. J. Phys. Chem. C 2009, 113, 1020-1027.

(134) Hallbäck, A.-S.; Poelsema, B.; Zandvliet, H. J. W. Negative Differential Resistance of TEMPO Molecules on Si(111). Appl. Surf. Sci. 2007, 253, 40664071. 
(135) Guisinger, N. P.; Elder, S. P.; Yoder, N. L.; Hersam, M. C. Ultra-High Vacuum Scanning Tunnelling Microscopy Investigation of Free Radical Adsorption to the Si(111)-7×7 Surface. Nanotechnology 2007, 18, 44011.

(136) Busolo, F.; Franco, L.; Armelao, L.; Maggini, M. Dynamics of a Nitroxide Layer Grafted onto Porous Silicon. Langmuir 2010, 26, 1889-1893.

(137) Skene, W. G.; Connolly, T. J.; Scaiano, J. C. Trapping of Photogenerated Group IV Radicals by TEMPO: Potential New Organometallic Initiators For "living" free Radical Polymerization. Int. J. Chem. Kinet. 2000, 32, 238-244.

(138) Lucarini, M.; Marchesi, E.; Pedulli, G. F.; Chatgilialoglu, C. Homolytic Reactivity of Group 14 Organometallic Hydrides toward Nitroxides. J. Org. Chem. 1998, 63, $1687-1693$.

(139) Chatgilialoglu, C.; Guerrini, A.; Lucarini, M.; Pedulli, G. F.; Carrozza, P.; Da Roit, G.; Borzatta, V.; Lucchini, V. Autoxidation of Poly(hydrosilane)s. Organometallics 1998, 17, 2169-2176.

(140) Chatgilialoglu, C.; Ferreri, C.; Vecchi, D.; Lucarini, M.; Pedulli, G. F. Free Radical Chemistry Associated with $\mathrm{H}(\mathrm{RSiH}) \mathrm{nH}$. J. Organomet. Chem. 1997, 545$546,475-481$.

(141) Matsumoto, A.; Ito, Y. New Generation of Organosilyl Radicals by Photochemically Induced Homolytic Cleavage of Silicon-Boron Bonds. J. Org. Chem. 2000, 65, 5707-5711.

(142) Gonzalez, C. M.; Iqbal, M.; Dasog, M.; Piercey, D. G.; Lockwood, R.; Klapötke, T. M.; Veinot, J. G. C. Detection of High-Energy Compounds Using Photoluminescent Silicon Nanocrystal Paper Based Sensors. Nanoscale Comm. 2014.

(143) Rehm, J. M.; Mclendon, G. L.; Fauchet, P. M. Conduction and Valence Band Edges of Porous Silicon Determined by Electron Transfer. J. Am. Chem. Soc. 1996, 118, 4490-4491.

(144) Kelly, M. T.; Bocarsly, A. B. Mechanisms of Photoluminescent Quenching of Oxidized Porous Silicon Applications to Chemical Sensing. Coord. Chem. Rev. 1998, 171, 251-259. 
Appendix

Supporting Information for Chapter 4

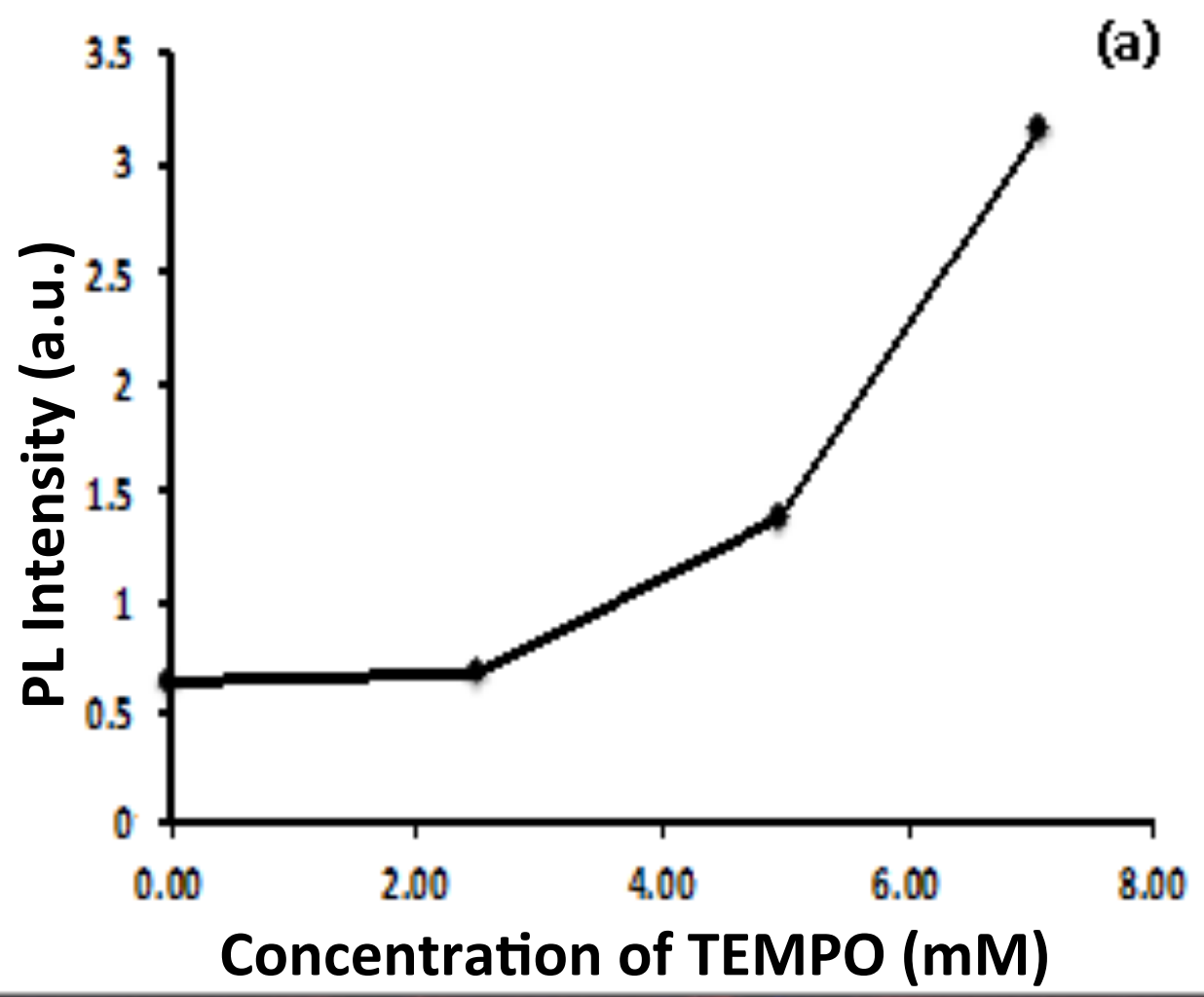

(b)

Figure A4. (a) Plot of $590 \mathrm{~nm}$ emission intensity versus concentration of TEMPO $\left(\lambda_{\text {exc }}=300 \mathrm{~nm}\right)$ added to $\mathrm{H}-\mathrm{Si} \mathrm{NP}$ suspensions. The TEMPO concentrations of the four samples (from left to right) were $0.0 \mathrm{mM}, 2.5 \mathrm{mM}$, $5.0 \mathrm{mM}$ and $7.1 \mathrm{mM}$. (b) Digital image of samples corresponding to data point 2, 3 and 4 (from left to right). Reprinted with permission from J. Phys. Chem. C, 2015, 119 (17), pp 9595-9608. Copyright 2015 American Chemical Society. 


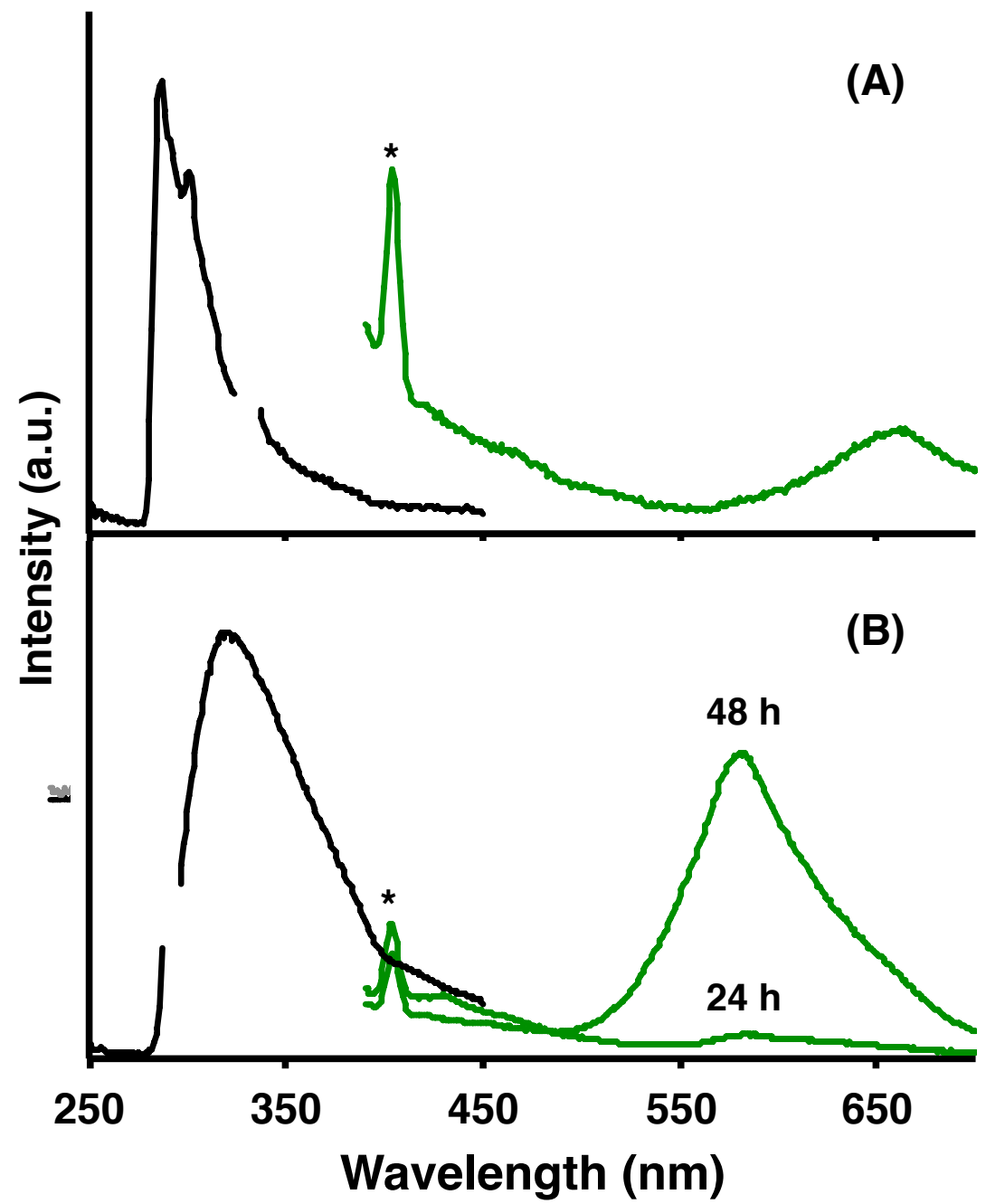

Figure A2. PL ( $\lambda_{\text {exc }} 360 \mathrm{~nm}$, green lines) spectra of (A) H-Si NPs and (B) scaled-up suspension of Si NPs and $8 \mathrm{mM}$ TEMPO $24 \mathrm{~h}$ and $48 \mathrm{~h}$ after sample preparation. PLE spectra (black lines) corresponding to (A) $660 \mathrm{~nm}$ and (B) $575 \mathrm{~nm}$ emissions. Breaks in data correspond to eliminated scattering peaks. $\left(^{*}\right)$ indicate peaks corresponding to light scattering by the sample. 